\title{
LATTICE DESIGN OF THE INTEGRABLE OPTICS TEST ACCELERATOR AND OPTICAL STOCHASTIC COOLING EXPERIMENT AT FERMILAB
}

\section{BY}

GENE KAFKA

Submitted in partial fulfillment of the requirements for the degree of Doctor of Philosophy in Physics in the Graduate College of the Illinois Institute of Technology

Chicago, Illinois

May 2015 
(c) Copyright by GENE KAFKA

May 2015 


\section{ACKNOWLEDGMENT}

I am enormously indebted to my adviser Dr. Daniel Kaplan, who has steered me though several projects, encouraged me to apply for the APC fellowship at Fermilab, written proposals on my behalf and who has shown me the importance of being a well rounded Ph.D. student. A special thanks to Dr. Gail Hanson who propelled me forward early on in my Ph.D. career.

I am deeply indebted to Dr. Alexander Valishev, who late in my Ph.D. career, took me under his wing with supreme guidance, enthusiasm and unending patience to tackle an exciting and novel project. I am grateful to Dr. Valeri Lebedev whose extensive work and insights allowed me to complete my thesis. Rarely is one lucky enough to stand directly on the shoulders of giants - thanks to the Accelerator Physics Center at Fermilab and the Center for Accelerator and Particle Physics at IIT for providing the opportunity.

I would like to thank Dr. Durga Rajaram and Dr. Pavel Snopok for the many days of help with software issues - their expertise and friendship have been enormously valuable. Thank you also to my committee members whom I have not yet singled out: Dr. Linda Spentzouris and Dr. Thomas Wong.

Thanks to my friends ${ }^{1}$ at Colby, Riverside, IIT, Fermilab and in Santa Monica.

A special thanks to my wife, Araceli Gallegos, who has endured with me the demands of this Ph.D., and who stands forever as my beacon of love and light.

My deepest gratitude is reserved for my parents Robert and Kyu-ja Kafka for their love and encouragement and for providing me with a wonderful life. My

${ }^{1}$ Special thanks to my fellow physicists: Omid Ahmadi, Heman Gharibnejad, Walter Hopkins, Kyle Keane, Alex Kozen, Michael Ng, Harold Nguyen, Kristoff Paulson, Ritoban Thakur and Gian Verri-Guzman. 
mother, who passed away months before the completion of this dissertation, has been the driving force behind many of my endeavors. The magnitude of her love and support is still easily felt in her absence. To her I dedicate this work, and in her enthusiastic spirit, I continue on to the next. 
TABLE OF CONTENTS

Page

ACKNOWLEDGEMENT . . . . . . . . . . . . . . . iii

LIST OF TABLES . . . . . . . . . . . . . . . . . v vii

LIST OF FIGURES . . . . . . . . . . . . . . . . . . . . .

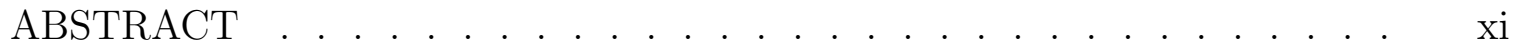

CHAPTER

1. INTRODUCTION AND MOTIVATION . . . . . . . . . . 1

1.1. Motivation for the Non-Linear Integrable Optics Test . . . 2

1.2. Motivation for Optical Stochastic Cooling . . . . . . . . 3

2. BEAM DYNAMICS . . . . . . . . . . . . . . 5

2.1. Coordinate System and Equations of Motion . . . . . 5

2.2. First and Second Order Optics Using Characteristic Rays . 12

2.3. Longitudinal Dynamics . . . . . . . . . . . . . . . . . 16

2.4. Equilibrium Emittance in a Storage Ring . . . . . . . . 20

2.5. Intra-beam Scattering and the Touschek Effect . . . . . . 22

2.6. Chromaticity . . . . . . . . . . . . . . . . . . 24

2.7. 2-D, 4-D and 6-D Beam Matching . . . . . . . . . . . 25

3. NONLINEAR INTEGRABLE OPTICS . . . . . . . . . . 29

3.1. Theory of Nonlinear Integrable Optics . . . . . . . . . . 29

4. LATTICE DESIGN FOR IOTA . . . . . . . . . . . . . 36

4.1. Parameters for the Nonlinear Integrable Optics Experiments 36

4.2. Dipoles and Quadrupoles . . . . . . . . . . . . . . . 38

4.3. Design of the Nonlinear Magnet Experiments . . . . . . . 41

4.4. Design of the electron lens Experiment . . . . . . . . . 46

4.5. Quadrupole Currents . . . . . . . . . . . . . . . . . . 48

4.6. RF Considerations . . . . . . . . . . . . . . 51

4.7. IOTA Parameters . . . . . . . . . . . . . . . . 52

5. OPTICAL STOCHASTIC COOLING IN IOTA . . . . . 57

5.1. Stochastic Cooling Theory . . . . . . . . . . . 57

5.2. OSC Bypass Optics . . . . . . . . . . . . . . . . 60

5.3. Path Lengthening in the Bypass . . . . . . . . . . . . . 65

5.4. Optics Control in the Bypass . . . . . . . . . . . 66 
5.5. Sextupole Placement in the Bypass . . . . . . . . . . . . 69

5.6. Optics Control in the Ring . . . . . . . . . . . . . . . 71

5.7. Second Order Path Length . . . . . . . . . . . . . . . 72

5.8. Sample Lengthening with the Polymorphic Tracking Code (PTC) Module in MadX . . . . . . . . . . . . . . . . 74

5.9. Further Reducing the Sample Lengthening . . . . . . . . . 79

5.10. Further Considerations for OSC optics . . . . . . . . . 87

5.11. OSC Simulation Studies _. . . . . . . . . . . . . 90

5.12. OSC Summary . . . . . . . . . . . . . . . . . . 91

6. CONCLUSION . . . . . . . . . . . . . . . . . 93

6.1. IOTA Lattice . . . . . . . . . . . . . . . . . . . 93

6.2. OSC Experiment . . . . . . . . . . . . . . . 94

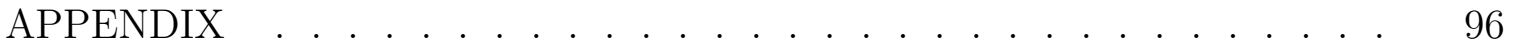

A. CHARACTERISTIC RAYS IN A MAGNETIC FIELD . . . . 96

B. PYTHON SCRIPT FOR THE IOTA GEOMETRY . . . . . . . 99

C. ENVELOPE QUANTITIES AND MATRIX ELEMENTS . . . . 105

D. MADX/PTC SCRIPT FOR OSC BYPASS SEXTUPOLES . . . 110

E. PYTHON/MADX SIMULATION FOR OSC . . . . . . . . 113

BIBLIOGRAPHY . . . . . . . . . . . . . . . . . . . . . . 121 


\section{LIST OF TABLES}

Table

Page

2.1 Matrix Elements for Beam Line Components . . . . . . . . . . . 15

2.2 Second order matrix elements for optical beam-line components . . 16

4.1 Electron Lens Beam Parameters for $2.5 \mathrm{MeV}$ Protons . . . . . . . 48

4.2 IOTA quadrupole gradients, currents and voltages . . . . . . . . . 50

4.3 IOTA Beam Parameters for $150 \mathrm{MeV}$ Electrons . . . . . . . . . . 53

5.1 OSC Beam Parameters . . . . . . . . . . . . . . . . . . . . 87 


\section{LIST OF FIGURES}

Figure

Page

2.1 An arbitrary particle trajectory shown with relation to a reference trajectory . . . . . . . . . . . . . . . . . . . 6

2.2 Phase space ellipse . . . . . . . . . . . . . . . . . . . . . . . 11

2.3 The path length differences for particles with different momenta in a magnetic bypass. . . . . . . . . . . . . . . . . . . . . . . .

2.4 Two-dimensional surface of a torus in the four-dimensional $x-x^{\prime}$ and $y$-y planes. . . . . . . . . . . . . . . . . . . . . . . . .

3.1 Particle trajectories and phase-space ellipses in the original and normalized variables. . . . . . . . . . . . . . . . . . . . . . . . .

3.2 A transverse cross-section of the nonlinear magnet with field singularities ......................

3.3 Horizontal and vertical beta functions . . . . . . . . . . . . . . 34

4.1 The IOTA ring layout with the location of 3 experiments . . . . . 37

$4.23 \mathrm{D}$ drawings of the $60^{\circ}$ (left) and $30^{\circ}$ (right) dipoles for the IOTA ring. . . . . . . . . . . . . . . . . . . . . . . . . . 39

4.3 Dipole corrector package and quadrupole arrangement . . . . . . 40

4.4 Dubna Quadrupoles . . . . . . . . . . . . . . . . . . . 41

4.5 Integrable optics lattice functions . . . . . . . . . . . . . . . . 43

4.6 IOTA lattice with two nonlinear magnet inserts . . . . . . . . . 44

4.7 IOTA lattice with one nonlinear magnet insert . . . . . . . . . . 47

4.8 IOTA lattice with electron lens insert . . . . . . . . . . . . 49

4.9 IOTA quadrupole families . . . . . . . . . . . . . . . . 54

4.10 RF Separatrix . . . . . . . . . . . . . . . . . 55

4.11 RF energy acceptance and synchrotron tune versus RF voltage. . . 56

5.1 Horizontal damping of betatron oscillations . . . . . . . . . . . 58

5.2 Heating and cooling contributions to stochastic cooling. The optimal gain is marked by the dotted line. . . . . . . . . . . . . . . 
5.3 The OSC bypass including two undulators, quadrupoles and bending magnets . . . . . . . . . . . . . . . . . .

5.4 Particle and radiation in an undulator segment. . . . . . . . . . 61

5.5 Electron interaction with radiation . . . . . . . . . . . . . . . . 62

5.6 Theoretical sample lengthening due to horizontal and momentum offset through the bypass . . . . . . . . . . . . . . . . . . 67

5.7 Beta functions of the OSC bypass . . . . . . . . . . . . . . . . 68

5.8 Sextupole kick . . . . . . . . . . . . . . . . . . . . . . . . . 70

5.9 The horizontal and vertical phase advance along with the horizontal dispersion in the bypass . . . . . . . . . . . . . . . . . . . . . 71

5.10 Bypass ray trajectory for the OSC experiment. . . . . . . . . . . 72

5.11 Dispersion invariant, $\mathcal{H}_{x}$, the dispersion, $D_{x}$, and the horizontal beta function $\beta_{x}$. . . . . . . . . . . . . . . . . . . . . . . . . . . 73

5.12 Matched beam for bypass particle tracking . . . . . . . . . . . . 75

5.13 RMS bunch length histograms in the bypass . . . . . . . . . . . 77

5.14 RMS path length difference in the bypass . . . . . . . . . . . . 78

5.15 Similar plot as Figure 5.14, but with a sextupole correction. . . . 80

5.16 The particle's longitudinal displacement versus its horizontal offset, without sextupoles in place. . . . . . . . . . . . . . . . . . .

5.17 The particle's longitudinal displacement versus its horizontal offset; with sextupoles in place. . . . . . . . . . . . . . . . . . 82

5.18 The uncorrected and corrected RMS sample lengthening are plotted versus the horizontal emittance and compared to the theoretical sample lengthening . . . . . . . . . . . . . . . . . . . . . . .

5.19 The uncorrected sample lengthening (18 cm dipoles) . . . . . . . 85

5.20 The corrected sample lengthening (18 cm dipoles) . . . . . . . . 86

5.21 The uncorrected sample lengthening vs. the horizontal position for a bypass with $18 \mathrm{~cm}$ dipoles . . . . . . . . . . . . . . . . . . . 88

5.22 The corrected sample lengthening for a bypass with $18 \mathrm{~cm}$ dipoles

5.23 The magnitude of each kick with respect to the particle's longitudinal offset on each revolution around the ring. 
5.24 A particle's coordinates in $z-\delta$ phase space in the OSC experiment

A.1 The cosine-like trajectory $C_{x}(s)$ in the bending plane . . . . . . . 97

A.2 The sine-like trajectory $S_{x}(s)$ in the bending plane . . . . . . . . 97

A.3 The dispersion-like trajectory $D_{x}(s)$ in the bending plane . . . . . 98

B.1 The IOTA ring layout generated with python . . . . . . . . . 100

C.1 The semi-major, $A$, and minor, $B$, axes of an ellipse tilted at an angle $\theta_{12}$ from the horizontal. . . . . . . . . . . . . . . . . . 108

C.2 Beam distribution with sigma cuts for PTC tracking . . . . . . . 109 


\begin{abstract}
The Integrable Optics Test Accelerator (IOTA) storage ring at Fermilab will serve as the backbone for a broad spectrum of Advanced Accelerator R\&D (AARD) experiments, and as such, must be designed with significant flexibility in mind, but without compromising cost efficiency. The nonlinear experiments at IOTA will include: achievement of a large nonlinear tune shift/spread without degradation of dynamic aperture; suppression of strong lattice resonances; study of stability of nonlinear systems to perturbations; and studies of different variants of nonlinear magnet design. The ring optics control has challenging requirements that reach or exceed the present state of the art. The development of a complete self-consistent design of the IOTA ring optics, meeting the demands of all planned AARD experiments, is presented. Of particular interest are the precise control for nonlinear integrable optics experiments and the transverse-to-longitudinal coupling and phase stability for the Optical Stochastic Cooling Experiment (OSC). Since the beam time-of-flight must be tightly controlled in the OSC section, studies of second order corrections in this section are presented.
\end{abstract}




\section{CHAPTER 1 \\ INTRODUCTION AND MOTIVATION}

Advances in accelerator technology have deepened physicists' understanding of the subatomic world for the past several decades. More recently, such technologies have also allowed biologists and chemists to see more clearly into the molecular world. The necessity for particle accelerators across multiple fields has created a demand for a variety of unique beam parameter sets. The Advanced Superconducting Test Accelerator (ASTA) at Fermilab's New Muon Lab (NML) [1] underwent the initial phases of construction in 2009 to carry out fundamental accelerator research and, since then, has been a point of interest for the wider Advanced Accelerator R\&D (AARD) community. ASTA will provide users with extremely stable beams, high repetition rate, and high average power at energies up to $\approx 0.3 \mathrm{GeV}$. It will include an Integrable Optics Test Accelerator (IOTA) — a small and flexible storage ring with innovative optics - which will be the backbone of a number of proof-of-principle beam physics experiments, including the nonlinear integrable optics test, optical stochastic cooling (OSC) experiment, and the development of space charge compensation in high intensity charged particle beams. ASTA will enable the study and exploration of new methods for overcoming intensity and energy limiting phenomena for high energy physics (HEP) discovery science. Its innovations will help enable the construction of a new generation of colliders and intensity frontier accelerators.

With the recent discovery of the Higgs boson at the Large Hadron Collider (LHC), lepton colliders are now desired to further investigate the Higgs boson. A muon collider is the only machine of this type with sufficient energy resolution to directly measure the predicted $4 \mathrm{MeV}$ width of the $126 \mathrm{GeV}$ standard-model Higgs boson. This $\mu^{+} \mu^{-}$"Higgs factory" will require a muon beam that has "cooled" position and momentum components - greatly reduced in magnitude - in both the 
transverse and longitudinal planes (six dimensions in total: $x, p_{x}, y, p_{y}, z, p_{z}$ ) in order to achieve the required energy spread and luminosity, something that will be challenging with current methods of cooling. OSC looks promising for 6D cooling in a proton collider and may be used for the LHC luminosity upgrade. If successful OSC is implemented in current machines, it may be plausible to extend such a technique to cool muons in a $\mu^{+} \mu^{-}$Higgs factory.

\subsection{Motivation for the Non-Linear Integrable Optics Test}

When building a storage ring, unavoidable magnetic errors are introduced into the system that drive the motion of the particles into resonances, which lead to instabilities. These resonances occur in a storage ring as particles periodically interact with elements that perturb their oscillations around the design orbit - the so-called "betatron motion." The number of these oscillations per revolution is called the tune. In current practice, to ensure beam stability and a sufficient lifetime, all resonances up to some high order must be avoided for all beam particles. This is possible only if the tune spread is kept extremely low (typically below 0.01 in units of $2 \pi$ ). Sextupole magnets are therefore placed in the lattice to correct the quadrupole focal lengths for off-energy particles. This correction is nonlinear (because it relies on the square of the particle's transverse offset), and as a result, the tune becomes dependent on the amplitude of the betatron motion. At high momentum deviations and large amplitudes, higher-order multipole terms dominate, and the efficiency of correcting magnets is diminished. Furthermore, random errors drive resonances, and higher-order ones can produce a gradual increase of particle amplitudes, leading to particle loss. This is problematic when considering the design of future high-energy and high-intensity machines. To achieve the "super-high" beam intensities that are desirable for future colliders, a tune spread of $\approx 50 \%$ of the central betatron tune is needed in order to allow strong Landau damping $[2,3]$. Landau damping is an 
effect that a driving oscillator has on the center of mass of a collection of particles with a large frequency distribution. Its consequences eliminate any coherent motion at infinitesimal amplitudes before it can grow. If the beam does not possess an adequate frequency spread, the beam size will grow exponentially; but for a large enough spread, the exponential growth will be suppressed, and the beam oscillation will be damped.

As discussed above, all nonlinearities in present storage rings lead to resonances and particle loss; however, there are some nonlinearities that can accommodate a large frequency spread while creating zero resonance strengths. We call these nonlinearities "integrable." The nonlinear integrable optics test at IOTA will attempt to circulate electrons in a storage ring without exciting resonances, thus obviating the need for higher-order correcting magnets, and making it possible to operate a storage ring with a large tune spread.

\subsection{Motivation for Optical Stochastic Cooling}

In 1972, Simon van der Meer proposed a method for damping betatron oscillations inside a storage ring [4]; he named this method "stochastic damping." His method seems to circumvent Liouville's well-known theorem, which states that the volume of a beam occupying a $6 \mathrm{D}$ phase space - having three spatial and three momentum coordinates $\left(x, y, t ; p_{x}, p_{y}, \Delta E\right)$-is an invariant. This theorem holds only if no information can be extracted about the location of individual particles in phase space; but if a particle's motion can be observed, a correction could be applied to damp the betatron oscillation. In this way, a pickup-kicker feedback system can damp coherent betatron oscillations (where the beam acts like a single particle). After one pass through this system, the beam would not be able to be damped further; however, the particles do not have the same revolution time (due to momentum spread) or betatron tunes. This has the effect of reintroducing randomness; as a consequence, 
new coherent motions are established on subsequent revolutions around the ring, and the beam can be further damped. For effective cooling, the pickup and kicker should sense and affect small samples of the total beam. This requires a large-bandwidth waveguide feedback system for effective coherent cooling and can be achieved with optical stochastic cooling (OSC). This method of cooling works on the same principles as van der Meer's method, but requires stricter control over the time of flight of the particles between the pickup and kicker. The OSC channel includes two undulators, an amplifier, and a bypass. The upstream undulator acts as a pickup whereby the particle bunch radiates an EM wave that propagates though an optical amplifier to the downstream undulator. Meanwhile, the bunch travels through a bypass to the second undulator where it is kicked by its own amplified radiation. In a finalized design, we expect to observe transverse and longitudinal cooling of an electron bunch within a few hundred turns around the ring. 


\section{CHAPTER 2}

\section{BEAM DYNAMICS}

In this chapter we first define the coordinate system for a charged particle beam in a storage ring. We then develop solutions to the second order differential homogenous equation that describes the linear motion of particles through magnetic elements around the storage ring, and parameterize these transformations using the oscillation phase of the particle, along with the geometric variables defined by the phase ellipse. We then return to a more general non-homogenous differential equation retaining transverse coordinates to second order, and, by using characteristic rays and Green's functions, develop first- and second-order transport matrices. Knowledge of the first-order transport matrices is needed for designing the linear lattice. The second-order transport matrices are necessary for correcting nonlinearities in the OSC bypass. The subsequent sections in the chapter deal with fundamental phenomena of an election storage ring.

\subsection{Coordinate System and Equations of Motion}

The motion of a charged particle through a beam line composed of magnetic fields is defined in a moving coordinate system centered on the reference trajectory. The coordinate $s$ is the arc length measured along this trajectory; the coordinates $x$, $y$ and $t$ make up a right-handed curvilinear coordinate system. It is common in accelerator physics to define the particle's phase space using the geometrical coordinates $\vec{X}$ as in [5]:

$$
\vec{X}(s)=\left(\begin{array}{c}
x \\
x^{\prime} \\
y \\
y^{\prime} \\
l \\
\delta
\end{array}\right),
$$




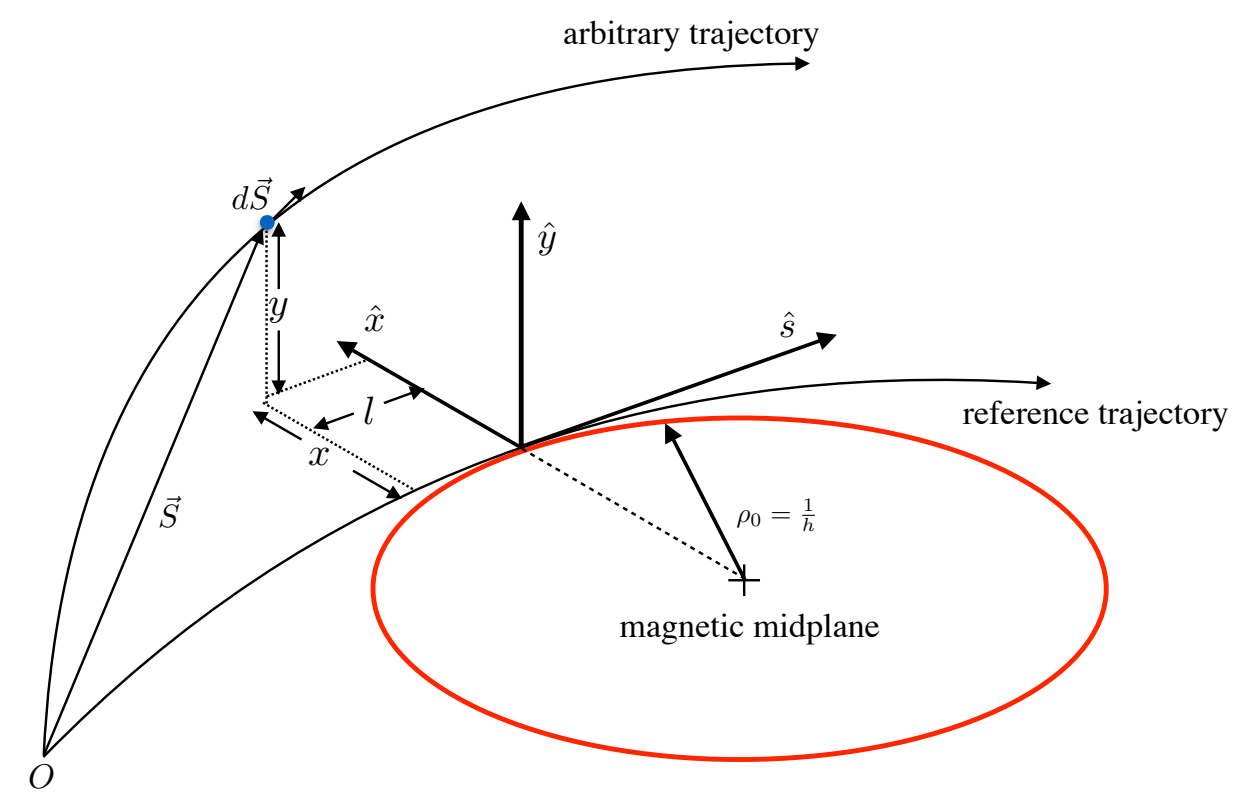

Figure 2.1. An arbitrary particle trajectory shown with relation to a reference trajectory which lies in the magnetic mid plane (after Ref. [5]).

where $x, y$ and $l$ are the horizontal, vertical and longitudinal displacements from the reference trajectory (see Figure 2.1); $\delta \equiv \Delta p / p$ is the relative momentum deviation from the design reference momentum. The coordinates $x^{\prime}=d x / d s$ and $y^{\prime}=d y / d s$ are the horizontal and vertical angles with respect to the reference trajectory (in the small-angle approximation). The effect upon the beam of any magnet in a beam line can be expressed with a $6 \times 6$ matrix, and when multiplied consecutively to include all magnets in the beam line, can transport any vector $\vec{X}(0)$ up or downstream to any arbitrary point $s$ as follows:

$$
\vec{X}(s)=R \vec{X}(0)
$$

where $R$ is the product of matrices between 0 and $s$. We can, for example, write the $x$ coordinate at some location as the linear combination of the matrix elements: $R_{11} x_{0}+R_{12} x_{0}^{\prime}+R_{16} \delta$, or more revealingly as:

$$
x=\left(x \mid x_{0}\right) x_{0}+\left(x \mid x_{0}^{\prime}\right) x_{0}^{\prime}+(x \mid \delta) \delta,
$$


keeping only terms which come from a midplane symmetric configuration as pictured in Figure 2.1, and where we have assumed no coupling between the transverse planes (i.e., $R_{13}=R_{14}=R_{23}=R_{24}=0$ ). The magnetic midplane in a ring with bending elements only in the horizontal plane lies perpendicular to the vertical, $y$, axis.

A storage ring is made up of a series of electromagnets (the "lattice") that shape the orbits of the charged particles and their bunches. The simplest type of ring is a weak focusing system and is made up of only dipole magnets which results in a relatively large beam size. For added stability, alternating quadrupole gradients can be added to create a strong focusing system. For any magnetic component in the lattice, the Lorentz force is used to characterize the time derivative of a particle's momentum, $\vec{p}$ while traversing a static magnetic field $\vec{B}$ :

$$
\dot{\vec{p}}=q(\vec{v} \times \vec{B})
$$

where $\vec{v}$ is the velocity vector, and $q$ is the charge of the particle. Since we have chosen a moving reference frame, to describe the trajectory we may eliminate time by formulating an equation of motion with respect the position vector $\vec{S}$ of a point along the particle's trajectory. Using $S$ as the distance along a particular trajectory, we can rewrite the momentum and velocity vectors $\vec{p}$ and $\vec{v}$ in terms of the unit vector $\frac{d \vec{S}}{d S}$ as

$$
p \frac{d \vec{S}}{d S} \text { and } v \frac{d \vec{S}}{d S}
$$

respectively. Equation 2.4 can then be re-written as

$$
v \frac{d}{d S}\left(\frac{d \vec{S}}{d S} p\right)=q v\left(\frac{d \vec{S}}{d S} \times B\right)
$$

where $v=\frac{d S}{d s}$. By differentiating the left side of the equation and noting that $\frac{d^{2} \vec{S}}{d S^{2}}$ is perpendicular to $\frac{d \vec{S}}{d S}$, it follows that $\frac{d p}{d S}=0$. We arrive at the final equation,

$$
\frac{d^{2} \vec{S}}{d S^{2}}=\frac{q}{p} \frac{d \vec{S}}{d S} \times \vec{B}
$$


while retaining complete generality. The orthogonal unit vectors $(\hat{x}, \hat{y}, \hat{s})$ describe a right-handed coordinate system and are diagramed in Figure 2.1. The origin, $O$, lies on the central trajectory. The positive direction of the $s$ coordinate is designated as the direction of motion of the particles on the central trajectory. The unit vectors satisfy the relations

$$
\begin{aligned}
& \hat{x}=\hat{y} \times \hat{s}, \\
& \hat{y}=\hat{s} \times \hat{x}, \\
& \hat{s}=\hat{x} \times \hat{y},
\end{aligned}
$$

where $\hat{s}$ points along the reference trajectory; $\hat{x}$ points radially out in the symmetry plane; and $\hat{y}$ points in the vertical direction perpendicular to the bending plane. We can now write the derivatives of each unit vector with respect to $s$ (denoted with primes) as:

$$
\begin{aligned}
& \hat{x}^{\prime}=h \hat{s}, \\
& \hat{y}^{\prime}=0, \\
& \hat{s}^{\prime}=-h \hat{x},
\end{aligned}
$$

where $h(s)=\frac{1}{\rho_{0}}$ is the curvature of the trajectory, and $\rho_{0}$ is the bending radius of the storage ring. The bending radius is easily calculated given the magnetic field $B$ (in tesla) and the design momentum $p_{0}($ in $\mathrm{GeV} / \mathrm{c})$ :

$$
\rho_{0}[\text { meters }]=\frac{B[\text { tesla }]}{3.3356 \times p_{0}[\mathrm{GeV} / \mathrm{c}]} .
$$

We can rewrite Eq. 2.7 making use of the curvilinear coordinates described above where

$$
\begin{aligned}
& \frac{d \vec{S}}{d S}=\frac{(d \vec{S} / d s)}{(d S / d s)}=\frac{\vec{S}^{\prime}}{S^{\prime}} \\
& \frac{d^{2} \vec{S}}{d S^{2}}=\frac{1}{S^{\prime}} \frac{d}{d s}\left(\frac{\overrightarrow{S^{\prime}}}{S^{\prime}}\right)
\end{aligned}
$$


Rearranging Eq. 2.12 and substituting it into Eq. 2.7, the equation of motion becomes

$$
\vec{S}^{\prime \prime}-\frac{1}{2} \frac{\vec{S}^{\prime}}{\left(S^{\prime}\right)^{2}} \frac{d}{d s}\left(S^{\prime}\right)^{2}=\frac{q}{p} S^{\prime}\left(\vec{S}^{\prime} \times \vec{B}\right)
$$

The differential line element along the trajectory in the curvilinear coordinate system is given by:

$$
(d S)^{2}=d \vec{S} \cdot d \vec{S}=d x^{2}+d y^{2}+(1+h x)^{2} d s^{2}
$$

We can differentiate Eq. 2.14 with respect to $s$ and obtain expressions for $S^{\prime}, \vec{S}^{\prime}$, and $\vec{S}^{\prime \prime}$ as outlined in Ref. [5]. Substituting these expressions into Eq. 2.13, and retaining only terms that are linear and quadratic in the trajectory coordinates, we arrive at the following equation of motion in the horizontal plane:

$$
x^{\prime \prime}-h(1+h x)-x^{\prime}\left(h x^{\prime}+h^{\prime} x\right)=\frac{q}{p} S^{\prime}\left[y^{\prime} B_{s}-(1+h x) B_{y}\right],
$$

where the subscripted $B$ denotes the magnetic field and its direction. From this expression, skipping several steps, the magnetic field in vacuum can be expressed in terms of a scalar potential which can be written as an infinite series; when we force the scalar potential to satisfy Laplace's equation, we obtain the following equations of motion (retaining terms to second order in horizontal coordinates) $[5,6]$ :

$$
\begin{aligned}
x^{\prime \prime}+(1-n) h^{2} x=h \delta+(2 n & -1-\beta) h^{3} x^{2}+h^{\prime} x x^{\prime}+\frac{1}{2} h x^{\prime 2}+(2-n) h^{2} x \delta \\
& +\frac{1}{2}\left(h^{\prime \prime}-n h^{3}+2 \beta h^{3}\right) y^{2}+h^{\prime} y y^{\prime}-\frac{1}{2} h y^{\prime 2}-h \delta^{2} .
\end{aligned}
$$

Here, the dimensionless parameters $n$ and $\beta$ are the first and second derivatives of the magnetic field, and are given by

$$
n=-\left[\frac{1}{h B_{y}} \frac{\partial B_{y}}{\partial x}\right]_{x=0, y=0} \text { and } \beta=\left[\frac{1}{2 h^{2} B_{y}} \frac{\partial^{2} B_{y}}{\partial x^{2}}\right]_{x=0, y=0}
$$

Since we have retained terms to second order in horizontal coordinates, the solution to Eq. 2.16 determines the form of the first- and second-order matrix elements, the latter of which will be covered in section 2.2 . 
We first consider the first order approximation of transporting particles through a magnetic field - with momentum deviation $\delta=0$ and assuming no high order aberrations - in which case Eq. 2.16 of the horizontal betatron motion simplifies to the homogeneous form of Hill's equation [7]:

$$
x^{\prime \prime}(s)+K_{x}^{2}(s) x(s)=0,
$$

where, in keeping with Eq. 2.16, $K_{x}^{2}(s)=(1-n) h^{2}$. This equation has solutions of the following form:

$$
\begin{aligned}
& x(s)=\sqrt{J_{x} \beta_{x}(s)} \cos \left(\mu_{x}\right) \\
& x^{\prime}(s)=-\sqrt{\frac{J_{x}}{\beta_{x}(s)}}\left[\sin \left(\mu_{x}\right)+\alpha(s) \cos \left(\mu_{x}\right)\right],
\end{aligned}
$$

where the invariant of the motion, $J_{x}$ (sometimes called the action), is constant and the phase function, $\mu_{x}$, parameterizes the oscillations of the betatron function, $\beta_{x}(s)$, in a given section of the ring and is given by

$$
\mu_{x}(s)=\int_{0}^{s} \frac{d s}{\beta_{x}(s)} .
$$

The number of these oscillations per turn is known as the betatron tune. By plugging Eqs. 2.19 into Hill's equation, we find that the beta function must fulfill the differential equation

$$
\frac{1}{2} \beta_{x}(s) \beta_{x}^{\prime \prime}(s)-\frac{1}{4} \beta_{x}^{\prime}(s)+K_{x}(s) \beta_{x}^{2}(s)=1 .
$$

Equations 2.19 can be combined to form the invariant of the motion $J_{x}$ :

$$
J_{x}=\gamma_{x}(s) x(s)^{2}+2 \alpha_{x}(s) x(s) x^{\prime}(s)+\beta_{x}(s) x^{\prime}(s)^{2},
$$

with the contractions $\alpha_{x}(s)$ and $\gamma_{x}(s)$ defined as

$$
\alpha_{x}(s)=-\frac{1}{2} \beta_{x}(s)^{\prime} \text { and } \gamma_{x}(s)=\frac{1+\alpha_{x}(s)^{2}}{\beta_{x}(s)} .
$$

Equation 2.22 describes an ellipse in $\left(x, x^{\prime}\right)$-phase space. The Twiss parameters $\left(\alpha_{x}, \beta_{x}\right.$ and $\gamma_{x}$ ) determine the shape and orientation of the ellipse (see Figure 2.2), which is 


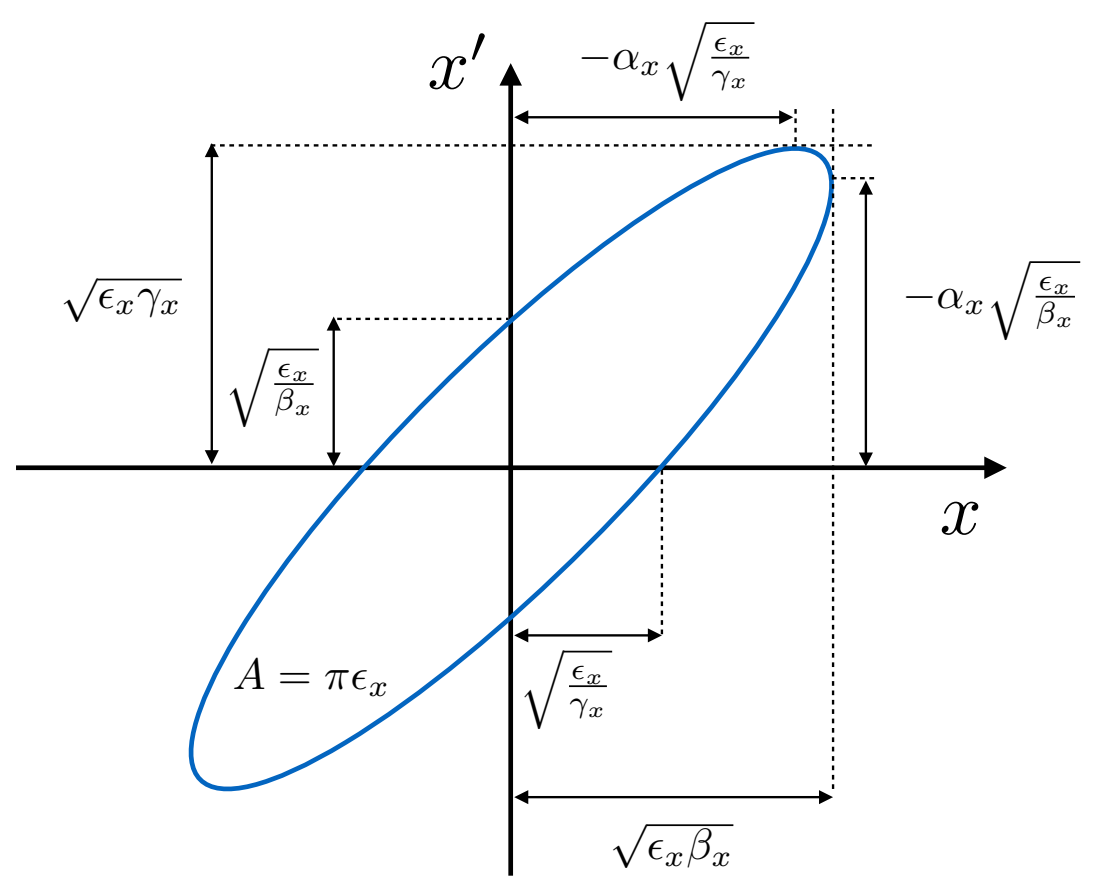

Figure 2.2. The phase space ellipse for a particle distribution with emittance $\epsilon_{x}$ at a given position in a storage ring, the dimensions of which are given in terms of the Twiss parameters. The phase space ellipse for a single particle is the same except that $\epsilon_{x}$ is replaced by the particle's action $J_{x}$.

different at every point in the accelerator, but whose area, $\pi J_{x}$, remains constant. A particle occupies a point around the circumference of the ellipse; its position moves along the ellipse with every revolution in the ring. If we consider only horizontal motion, described by Eqs. 2.19, the matrix in Eq. 2.3 can be written to transport the horizontal vector from point $s_{0}$ through any number of accelerator components to any location, $s$, in terms of the initial and final Twiss parameters and the phase advance - defined as the difference of the phase at point $s$ and some initial point 0 - which is denoted by $\mu_{s_{0} s}=\psi(s)-\psi(0)$ :

$$
\left(\begin{array}{c}
x(s) \\
x^{\prime}(s)
\end{array}\right)=\left(\begin{array}{cc}
\sqrt{\frac{\beta(s)}{\beta_{0}}\left[\cos \mu_{s_{0} s}+\alpha_{0} \sin \mu_{s_{0} s}\right]} & \sqrt{\beta_{0} \beta(s)} \sin \mu_{s_{0} s} \\
\frac{-\left[\alpha(s)-\alpha_{0}\right] \cos \mu_{s_{0} s}+\left[1+\alpha_{0} \alpha(s)\right] \sin \mu_{s_{0} s}}{\sqrt{\beta_{0} \beta(s)}} & \sqrt{\frac{\beta_{0}}{\beta(s)}}\left[\cos \mu_{s_{0} s}-\alpha \sin \mu_{s_{0} s}\right]
\end{array}\right)\left(\begin{array}{c}
x(0) \\
x^{\prime}(0)
\end{array}\right) .
$$


We will now expand our treatment of the motion of a single particle to describe an entire bunch of particles occupying the same phase space. The betatron function relates the transverse RMS size of the beam $\left(\sigma_{x}(s)\right)$ and its divergence $\left(\sigma_{x^{\prime}}(s)\right)$ to the invariant emittance area, $\epsilon_{x}$, in the horizontal plane:

$$
\begin{aligned}
\sigma_{x}(s) & =\sqrt{\epsilon_{x} \beta_{x}(s)} \\
\sigma_{x^{\prime}}(s) & =\sqrt{\epsilon_{x} \gamma_{x}(s)},
\end{aligned}
$$

where $\epsilon_{x}=\gamma_{x}\left\langle x^{2}\right\rangle+2 \alpha_{x}\langle x\rangle\left\langle x^{\prime}\right\rangle+\beta_{x}\left\langle x^{\prime 2}\right\rangle$. Figure 2.2 shows the phase space ellipse in terms of the Twiss parameters and the emittance.

In a storage ring, dispersion in the beam is generated in each dipole magnet. The dispersion function, $D_{x}$, describes a particle's acquired horizontal deviation due to its momentum offset as it travels through a dipole field. The linear dependence of the dispersion can be controlled with the optics of the lattice. In the presence of dispersion in the bending plane, the beam size and its angular divergence are defined as

$$
\begin{aligned}
\sigma_{x} & =\sqrt{\epsilon_{x} \beta_{x}(s)+\left(D_{x}(s) \delta\right)^{2}} \\
\sigma_{x^{\prime}} & =\sqrt{\epsilon_{x} \gamma(s)+\left(D_{x}^{\prime}(s) \delta\right)^{2}} .
\end{aligned}
$$

With dispersion present, the phase space ellipse is no longer centered at the origin; rather it is offset by $D_{x}(s) \delta$ and $D_{x}^{\prime}(s) \delta$ along the horizontal and vertical axes respectively.

\subsection{First and Second Order Optics Using Characteristic Rays}

The preceding sections are sufficient for understanding the motion of a particle in a linear beam line or circular accelerator. However, a more precise description of the beam dynamics requires the addition of higher order terms in the matrix expansion of all accelerator components by retaining all elements in the equations of motion (Eq. 2.16) to second order. For the nonlinear design of the OSC bypass we will need 
knowledge of the second order matrix elements and their effect on the path lengthening of a particle's trajectory. This lengthening can be corrected with nonlinear elements such as sextupoles. When sextupoles are properly placed in the bypass, they can be tuned to cancel the second order matrix elements that contribute most to path lengthening. Here we develop the first order matrix elements using characteristic rays and Green's functions, and extend the same method to find the second order matrix elements.

The linear treatment for transporting a particle's horizontal position as presented in Eq. 2.3 can be extended to include additional terms of second order as follows:

$$
\begin{aligned}
x= & \left(x \mid x_{0}\right) x_{0}+\left(x \mid x_{0}^{\prime}\right) x_{0}^{\prime}+(x \mid \delta) \delta+\left(x \mid x_{0}^{2}\right) x_{0}^{2}+\left(x \mid x_{0} x_{0}^{\prime}\right) x_{0} x_{0}^{\prime}+\left(x \mid x_{0} \delta\right) x_{0} \delta \\
& +\left(x \mid x_{0}^{\prime 2}\right) x_{0}^{\prime 2}+\left(x \mid x_{0}^{\prime} \delta\right) x_{0}^{\prime} \delta+\left(x \mid \delta^{2}\right) \delta^{2}+\left(x \mid y_{0}^{2}\right) y_{0}^{2}+\left(x \mid y_{0} y_{0}^{\prime}\right) y_{0} y_{0}^{\prime}+\left(x \mid y_{0}^{\prime 2}\right) y_{0}^{\prime 2} .
\end{aligned}
$$

The first three terms are of first order, and make up the matrix elements $R_{i j}$; the remaining terms are contained in a second order matrix and are given by $T_{i j k}$. A combination of first and second order matrices transports the vector from point 0 to point 1:

$$
x_{i}(1)=\sum_{j}^{6} R_{i j} x_{j}(0)+\sum_{j}^{6} \sum_{k}^{6} T_{i j k} x_{j}(0) x_{k}(0),
$$

where $x_{1}=x, x_{2}=\theta, x_{3}=y, x_{4}=\phi, x_{5}=l$, and $x_{6}=\delta ; \theta$ and $\phi$ are the horizontal and vertical divergences from the reference trajectory.

When developing second order transfer matrices, we must revisit Eq. 2.16. This equation of motion is a second order inhomogeneous differential equation. We geometrically define three characteristic rays (see Appendix A) that represent the solutions to the homogeneous equation (Eq. 2.18) and one characteristic ray that represents the solution to the inhomogeneous (Eq. 2.16) equation: 
1. the unit sine-like ray in the bending plane $S_{x}(s)$ that is coincident with the central trajectory at the end points;

2. the unit cosine-like ray in the bending plane $C_{x}(s)$ that is off the central trajectory at the end points;

3. the dispersion ray in the bending plane $D_{x}(s)$ that diverges from the central trajectory;

The general solution to the differential equation for $x$ (Eq. 2.27) can now be written as a linear combination of these particular solutions and the second order terms [5]:

$$
x(s)=x_{0} C_{x}(s)+x_{0}^{\prime} S_{x}(s)+\delta D_{x}(s)+\text { second order terms }
$$

where $C_{x}(s), S_{x}(s)$ and $D_{x}(s)$ denote the sine-, cosine- and dispersion-like rays. A similar expression exists for the vertical plane which makes use of two additional characteristic rays not in the bending plane - for the sake of brevity we will omit it here. Substituting Eq. 2.29 into the equations of motion (Eq. 2.16), and equating coefficients of like products of the initial coordinates, we end up with a set of differential equations:

$$
\begin{aligned}
C_{x}^{\prime \prime}(s)+K_{x}^{2}(s) C_{x}(s) & =0 \\
S_{x}^{\prime \prime}(s)+K_{x}^{2}(s) S_{x}(s) & =0 \\
T_{i j k}^{\prime \prime}(s)+K_{x}^{2}(s) T_{i j k}(s) & =f_{i j k}(s) \\
D_{x}^{\prime \prime}(s)+K_{x}^{2} D_{x}(s) & =f_{\delta}(s) .
\end{aligned}
$$

The first two equations are the first-order equations of motion, and are different only by the initial conditions defined by the characteristic rays. It is straightforward to obtain the matrix elements for various beam-line components where $n, h$ and $\beta$ are constants over the interval of integration; the first order matrix elements are shown in Table 2.1. The third equation is for the motion governed by any of the second order elements $T_{i j k}$; the fourth is for the motion including effects from the first order 
dispersion $D_{x}$. The driving term $f_{i j k}$ is analogous to that seen in the equations of motion for a forced harmonic oscillator found in Eq. 2.16; it is written explicitly on the RHS of the equation and is a function of $n, \beta, h$ and their derivatives. The driving terms that are only proportional to the horizontal offset, namely $f_{111}=\left(x \mid x_{0}^{2}\right)$, are given by

$$
f_{111}=(2 n-1-\beta) h^{3} C_{x}^{2}+h^{\prime} C_{x} C_{x}^{\prime}+\frac{1}{2} h C_{x}^{\prime 2},
$$

where the characteristic ray $C_{x}$ is the ray that describes the horizontal offset, $x$. Using the boundary conditions for each characteristic ray outlined in Appendix A, the coefficients $T_{i j k}$ in Eqs. 2.30 are evaluated using a Green's function integral so that

$$
T_{i j k}=\int_{0}^{s} G(s, \tilde{s}) f_{i j k}(\tilde{s}) d \tilde{s} \quad ; \quad i=1,2,3,4 ; \quad j, k=1,2,3,4,5,6,
$$

where $\tilde{s}$ is a dummy variable, and the Green's function is given by $G(\tilde{s})=S_{x}(s) C_{x}(\tilde{s})-$ $S_{x}(\tilde{s}) C_{x}(s)[5,6]$. Table 2.2 includes the form of the integral for the second order matrix element $T_{111}$ as well as the longitudinal elements (to be discussed next); the rest of the elements which are omitted here, are derived in $[5,6]$.

Table 2.1. Matrix Elements for Beam Line Components

\begin{tabular}{cccccc}
\hline \hline Element & Name & Drift & Quad & Dipole & Thin Lens \\
\hline$R_{11}$ & $C_{x}(s)$ & 1 & $\cosh (h \sqrt{n} s)$ & $\cos (h s)$ & 1 \\
$R_{12}$ & $S_{x}(s)$ & $s$ & $\sinh (h \sqrt{n} s)$ & $\sin (h s)$ & 1 \\
$R_{21}$ & $C_{x}^{\prime}(s)$ & 0 & $-\sinh (h \sqrt{n} s)$ & $-\sin (h s)$ & 1 \\
$R_{22}$ & $S_{x}^{\prime}(s)$ & 1 & $\cosh (h \sqrt{n} s)$ & $\cos (h s)$ & 1 \\
\hline
\end{tabular}

The longitudinal matrix elements $T_{5 j k}$ are of particular interest in designing the OSC bypass and are briefly described here. To find these matrix elements, we begin with the general expression for the path length difference of a particle with respect to the reference trajectory:

$$
l=\int_{0}^{s}\left\{\left[\left(\frac{d x}{d \tilde{s}}\right)^{2}+\left(\frac{d y}{d \tilde{s}}\right)^{2}+(1+h x)^{2}\right]^{1 / 2}-1\right\} d \tilde{s},
$$


and using the binomial theorem to expand the square root, we have

$$
l=\int_{0}^{s}\left\{h x(\tilde{s})+\frac{1}{2}\left[\left(\frac{d x}{d \tilde{s}}\right)^{2}+\left(\frac{d y}{d \tilde{s}}\right)^{2}\right]\right\} d \tilde{s} .
$$

Considering only first order, we see that only the first term inside the integral contributes. The first-order matrix elements for the longitudinal coordinate $l$ then come from the first-order matrix elements for $h(x)$ (i.e., $h\left(x \mid x_{0}\right), h\left(x \mid x_{0}^{\prime}\right)$ and $h\left(x \mid \delta_{0}\right)$ along with terms due to the vertical motion). The second order matrix elements for $l$ come from the second order matrix elements for $h(x)$ (i.e., $h\left(x \mid x_{0}^{2}\right), h\left(x \mid x_{0} x_{0}^{\prime}\right), h\left(x \mid x_{0}^{\prime 2}\right)$, $h(x \mid x \delta), h\left(x \mid x^{\prime} \delta\right), h\left(x \mid \delta^{2}\right)$ and terms due to the vertical motion), and from the two derivatives in the brackets. We list several of the $T_{5 j k}$ integrals and their evaluations in Table 2.2 [6].

Table 2.2. Second order matrix elements for optical beam-line components

\begin{tabular}{cccccc}
\hline \hline Element & Integral & Drift & Quad & Dipole & Sextupole \\
\hline$T_{111}$ & $(2 n-1-\beta) h^{3} \times$ & 0 & 0 & $\frac{-k \sin ^{2}(k s)}{2}$ & $\frac{-K_{2} s^{2}}{4}$ \\
& $\int_{0}^{s}\left[C_{x}^{2}(\tilde{s}) G(\tilde{s}, s)\right] d \tilde{s}$ & 0 & 0 & 0 & 0 \\
& $+\frac{1}{2} h \int_{0}^{s}\left[S_{x}^{2}(\tilde{s}) G(\tilde{s}, s)\right] d \tilde{s}$ & & & \\
$T_{511}$ & $\int_{0}^{s}\left[h\left(x \mid x_{0}^{2}\right)+\frac{1}{2} C_{x}^{\prime 2}\right] d \tilde{s}$ & 0 & $-\frac{1}{4} K_{1}\left(s-\frac{\cos \left(\sqrt{K_{1}} s\right) \sqrt{K_{1}} \sin \left(\sqrt{K_{1}} s\right)}{\sqrt{K_{1}}}\right)$ & 0 & 0 \\
$T_{521}$ & $\int_{0}^{s}\left[h\left(x \mid x_{0} x_{0}^{\prime}\right)+C_{x}^{\prime} S_{x}^{\prime}\right] d \tilde{s}$ & 0 & $\frac{1}{2} \sin ^{2}\left(\sqrt{K_{1}} s\right)$ & 0 & 0 \\
$T_{522}$ & $\int_{0}^{s}\left[h\left(x \mid x_{0}^{\prime 2}\right)+\frac{1}{2} S_{x}^{\prime 2}\right] d \tilde{s}$ & $-\frac{s}{2}$ & $-\frac{1}{4}\left(s+\frac{\cos \left(\sqrt{K_{1}} s\right) \sqrt{K_{1}} \sin \left(\sqrt{K_{1}} s\right)}{\sqrt{K_{1}}}\right)$ & $\frac{-\sin (h s)}{2 h}$ & $\frac{-s}{2}$ \\
\hline
\end{tabular}

Here we have used the normalized quadrupole and sextupole coefficients: $K_{1}=$ $-k^{2} n$ and $K_{2}=k^{3} \beta$, where $k$ is the magnetic field strength.

\subsection{Longitudinal Dynamics}

Understanding the longitudinal dynamics of an electron bunch is critical for the design of IOTA and the OSC experiment. The stability of an electron bunch in a storage ring is dictated by the interplay of the energy lost and gained in the longitudinal plane via synchrotron radiation and RF cavities respectively. The precise 
control of the longitudinal dynamics in the OSC experiment is necessary for efficient cooling.

An important property of the magnetic focusing lattice is the momentum compaction $\alpha_{p}$. In a storage ring, $\alpha_{p}$ relates a particle's momentum to its closed orbit path length-particles with higher energies follow orbits larger than the reference orbit, mutatis mutandis - with the interplay between these two effects on the travel time around the ring determining the magnitude of compression of the longitudinal emittance of the beam. If we consider an off-momentum particle in a storage ring with momentum $p=p_{0}(1+\delta)$, where $p_{0}$ and $\rho_{0}$ are the design momentum and radius (as given previously in Eq. 2.10), it will execute revolutions at a radius greater or smaller than the design radius such that $\rho=\rho_{0}+\Delta \rho$. The revolution period, $T=\frac{2 \pi \rho}{v}$, for some velocity $v$ will also be different than that of an on-momentum particle. We define the momentum compaction as

$$
\alpha_{p}=\frac{\Delta T / T_{0}}{\delta}
$$

where $T_{0}$ is the revolution period of the reference particle. The momentum compaction can also be thought of as longitudinal dispersion. Now we can readily find the relative change in revolution time due to the change in radius and velocity of a particle:

$$
\frac{\Delta T}{T_{0}}=\frac{\Delta \rho}{\rho_{0}}-\frac{\Delta v}{v_{0}}=\left(\alpha_{p}-\frac{1}{\gamma^{2}}\right) \frac{\Delta p}{p_{0}}=\eta \delta,
$$

where $\eta=\left(\alpha_{p}-\frac{1}{\gamma^{2}}\right)$ is commonly known as the slip factor. In the case of highly relativistic particles, the slip factor is simply the momentum compaction. From Eq. 2.36, we see that at a particular design energy, where $\gamma_{0}=\frac{1}{\sqrt{\alpha_{p}}}$, the longitudinal dispersion, $\eta$, vanishes. This particular energy is called the "transition energy." When the design energy is above the transition energy, particles with positive momentum deviation lag behind the reference particle; and when the design energy is below the transition energy, particles with positive momentum deviation advance ahead of the reference 
particle. For storage rings with negative momentum compaction, low-momentum particles have longer closed orbits than high-momentum particles, which will in turn elongate the bunch.

The momentum compaction can also be expressed as the integrated product of the curvature $h(s)$ and the dispersion $D_{x}(s)$ of the reference trajectory:

$$
\alpha_{p}=\frac{1}{\Delta s} \int h(s) D_{x}(s) d s
$$

We see here that the momentum compaction is determined mainly by the dispersion where the curvature is high, i.e., in the dipoles. A momentum compaction that is either too large or small can be compensated by the RF system in the ring.

2.3.1 Magnetic Chicanes and Bypasses. As a slight diversion from the flow of this exposition, we now briefly explain the slip factor in a magnetic chicane, or bypass. A chicane is comprised of at least three bending magnets, the middle one being either twice the length or strength of the outer two. Figure 2.3 has four bending magnets all with the same strength and size. Chicanes in accelerators are typically used to manipulate the longitudinal phase space of a charged particle bunch by exploiting the different path lengths taken by particles of different momenta as seen in Figure 2.3. If a bunch has a suitably correlated longitudinal phase space at the beginning of the chicane, it can either be compressed or lengthened at the exit. In the case of the OSC bypass however, there will be no such correlation at the beginning of the bypass. We will attempt to preserve the different path lengths taken by particles of different momenta and cancel any higher order aberrations that would decouple the two, namely the aberration that is generated by the initial horizontal divergence going into the bypass.

2.3.2 Synchrotron Motion and RF Cavities. The energy change per turn supplied by an RF cavity with voltage $U$, and phase $\phi_{0}=h \omega_{0} t_{0}$ (where $h$ is the 


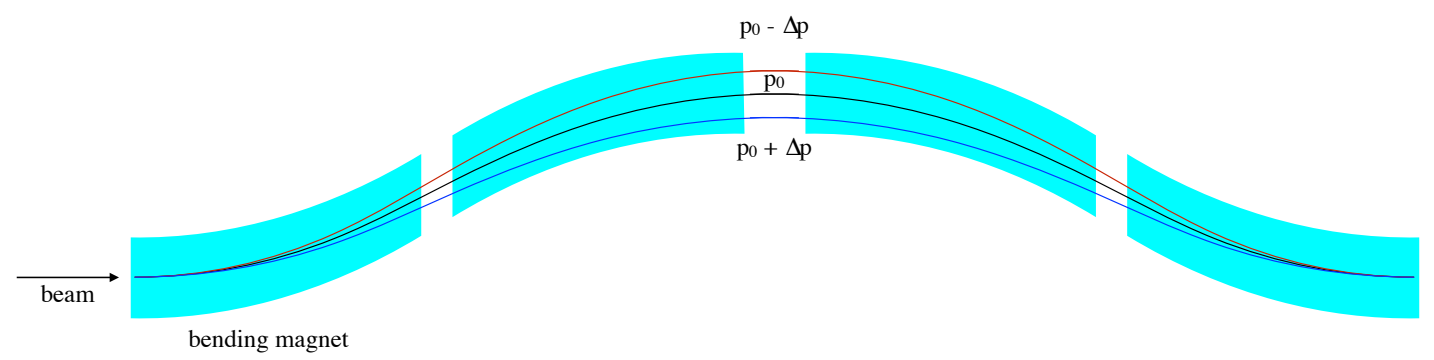

Figure 2.3. The path length differences for particles with different momenta in a magnetic bypass.

harmonic number, $\omega_{0}$ is the angular revolution frequency, and $t_{0}$ is the transit time for a synchronous particle) is simply:

$$
\Delta E=e U \sin \left(h \omega_{0}\left(t_{0}+\tau\right)\right)
$$

where $\tau$ is the longitudinal offset $z$ from the reference particle, divided by $c$. Typically, the damping time in a storage ring due to synchrotron motion is slow, so we can write the time derivative of the energy as

$$
\frac{d E}{d t}=\frac{\Delta E}{\Delta t}=\frac{\Delta E}{T}=\frac{e U}{T} \sin \left(h \omega_{0}\left(t_{0}+\frac{z}{c}\right)\right),
$$

where $T$ is the revolution period. The particle energy offset due to momentum deviation is $E=E_{0}+\epsilon$ where $\epsilon=\gamma m v^{2} \delta$. With this substitution, equation 2.39 becomes:

$$
\gamma m v^{2} \frac{d \delta}{d t}=\frac{e U}{T} \frac{\omega_{0}}{c} \cos \phi_{0} \cdot z
$$

Here, we have used the linear approximation of the sine function since the longitudinal offset of a particle is much smaller than the RF wavelength, $z<<\lambda_{R F}$; using a trigonometric identity lets us separate out the argument of the sine. Using 2.36, we obtain two time derivatives:

$$
\begin{aligned}
& \frac{d \delta}{d t}=\frac{e U}{T E_{0}} h \frac{\omega_{0}}{c} \cos \left(\phi_{0}\right) \cdot z \\
& \frac{d z}{d t}=\eta c \delta
\end{aligned}
$$


The right-hand side of Eq. 2.41a contains the expression for the synchrotron tune, $\nu_{s}$, which is the number of synchrotron oscillations in every revolution around the storage ring; it is given by

$$
\omega_{s}=\nu_{s} \omega_{0}=\omega_{0} \sqrt{-\frac{e U h}{2 \pi E_{0}} \cos \phi_{0} \eta} .
$$

Taking another time derivative of Eq. 2.41b and substituting Eq. 2.41a, we have:

$$
\frac{d^{2} z}{d t^{2}}=\nu_{s}^{2} \omega_{0}^{2} z=0 .
$$

In the absence of a driving term, the longitudinal equation of motion describes simple harmonic oscillations. The solutions for the longitudinal synchrotron motion are

$$
\left(\begin{array}{c}
z \\
(\Delta p / p)
\end{array}\right)=\left(\begin{array}{cc}
\cos \mu_{s} & \frac{\eta c}{\omega_{s}} \sin \mu_{s} \\
-\frac{\omega_{s}}{\eta c} \sin \mu_{s} & \cos \mu_{s}
\end{array}\right)\left(\begin{array}{c}
z_{0} \\
(\Delta p / p)_{0}
\end{array}\right) .
$$

Later, in Chapter 4, we will use these solutions to find the RF voltage and harmonic number needed for an appropriate RF acceptance in the ring.

\subsection{Equilibrium Emittance in a Storage Ring}

In an electron storage ring, synchrotron radiation plays a role in cooling the beam. As the electron trajectories are bent through the dipoles, the electrons radiate energy in the form of light in the direction of their motion and with a power proportional to the square of their energy, i.e., faster electrons lose more energy than slower electrons. When the electrons pass through RF cavities, their momentum is restored only longitudinally, thus the beam is cooled. The energy lost by a particle through synchrotron radiation on each turn around the ring can be estimated using the classical result that the power radiated by a particle of charge $e$ and energy $E_{0}$ in a magnetic field $B$ is $[8]$

$$
P_{\gamma}=\frac{C_{\gamma}}{2 \pi} c^{3} e^{2} B^{2} E_{0}^{2},
$$


where $C_{\gamma}=\frac{8.85 \times 10^{-5} \mathrm{~m}}{\mathrm{GeV}^{3}}$ for electrons. Using the equation for the beam rigidity $B \rho=$ $\frac{E_{0}}{e c}$, and, since for an ultra-relativistic electron, $E_{0}=p_{0} c$, we can rewrite the radiated power as

$$
P_{\gamma}=\frac{C_{\gamma}}{2 \pi} c \frac{E_{0}^{4}}{\rho^{2}}
$$

where $\rho$ is the bending radius. And finally, integrating around the ring, we come to the energy loss $U_{0}$ around the ring:

$$
U_{0}=\oint P_{\gamma} d t=\oint P_{\gamma} \frac{d s}{c}=\frac{C_{\gamma} E_{0}^{4}}{2 \pi} \oint \frac{1}{\rho^{2}} d s=\frac{C_{\gamma} E_{0}^{4}}{2 \pi} I_{2} .
$$

Here, we have used the second radiation integral ${ }^{2} I_{2}=\oint \frac{1}{\rho^{2}} d s$. The horizontal, vertical and longitudinal emittances decay exponentially such that

$$
\frac{d \epsilon_{i}}{d t}=-\frac{2}{\tau_{i}} \epsilon_{i} \quad ; \quad i=1,2,3
$$

where $i$ indicates each degree of freedom and where the damping times $\tau_{i}$ are dependent on the damping partition numbers $j_{i}$ :

$$
\tau_{i}=\frac{2}{j_{i}} \frac{E_{0}}{U_{0}} T_{0}
$$

The sum of the damping partition numbers is invariant: $j_{x}+j_{y}+j_{z}=4[9,10]$. The sum of the inverse damping times can then be calculated:

$$
\frac{1}{\tau_{x}}+\frac{1}{\tau_{y}}+\frac{1}{\tau_{z}}=\frac{U_{0}}{2 E T_{0}}\left(j_{x}+j_{y}+j_{z}\right)=\frac{2 U_{0}}{E_{0} T_{0}} .
$$

The individual damping decrements can be written in terms of synchrotron radiation integrals:

$$
j_{x}=1-\frac{I_{4}}{I_{2}}, \quad j_{y}=1, \quad j_{z}=2+\frac{I_{4}}{I_{2}},
$$

where the fourth synchrotron radiation integral is

$$
I_{4}=\oint \frac{D_{x}}{\rho}\left(\frac{1}{\rho^{2}}+2 K_{1}\right) d s
$$

${ }^{2}$ There are five commonly used synchrotron radiation integrals, all inversely proportional to different powers of the bending radius. 
where $K_{1}=\frac{q}{p_{0}} \frac{\partial B_{y}}{\partial x}$ is the quadrupole component of the magnetic field in the ring.

Radiation emission is a quantum process whereby photons are emitted with discrete energies. If this emission process were smooth, the emittance of the beam would damp to zero; however this quantum effect hinders the damping process, and creates "noise" on the beam. The beam reaches an equilibrium emittance when radiation damping balances the heating due to this quantum excitation. The rate of change of emittance can then be written as the combination of these two processes:

$$
\frac{d \epsilon_{x}}{d t}=-\frac{2}{\tau_{x}} \epsilon_{x}+\frac{2}{j_{x} \tau_{x}} C_{q} \gamma^{2} \frac{I_{5}}{I_{2}}
$$

where the "quantum" constant is $C_{q}=\frac{55}{32 \sqrt{3}} \frac{\hbar}{m c} \approx 3.832 \times 10^{-13} \mathrm{~m}$. By setting the left-hand side of the equation to zero, we can find the equilibrium emittance

$$
\epsilon_{0}=C_{q} \frac{\gamma^{2} I_{5}}{j_{x} I_{2}}
$$

where the fifth synchrotron integral is

$$
I_{5}=\oint \frac{\mathcal{H}_{x}}{\left|\rho^{3}\right|} d s
$$

$\mathcal{H}_{x}(s)$ is commonly known as the "dispersion invariant"; it is constant in drifts and in quadrupoles, but changes in bends. It is given by

$$
\mathcal{H}_{x}=\gamma_{x}(s) D_{x}(s)^{2}+2 \alpha_{x}(s) D_{x}(s) D_{x}^{\prime}(s)+\beta_{x}(s) D_{x}^{\prime}(s)^{2}
$$

Since $\mathcal{H}_{x}(s)$ increases the equilibrium emittance in the ring, if we wish to decrease the emittance, we can do so by decreasing $D_{x}(s)$ around the ring. When it comes to designing the ring optics for the OSC configuration, we adjust the dispersion and quadrupole gradients such that $\mathcal{H}_{x}(s)$ is minimized.

\subsection{Intra-beam Scattering and the Touschek Effect}

As with quantum fluctuations, there are other mechanisms that work against synchrotron damping to increase the size of the beam and lead to particle loss. For 
low-emittance beams, intra-beam scattering (IBS) is a severely limiting process. IBS accounts only for small-angle scattering that increases the emittance, but does not lead to particle loss. Touschek scattering accounts for large-angle scatters that kick particles outside the momentum acceptance such that they are quickly lost.

2.5.1 Approximation of the Touschek Effect. The Touschek effect is a process whereby particles within a bunch transfer momentum from the transverse to the longitudinal plane by large angle Coulomb collisions. The change of direction due to these collisions strongly affects the particle's energy via relativistic effects; such momentum redistributions degrade the dynamic aperture and are one of the restricting mechanisms in electron storage rings. Large angle collisions occur when the electron's amplitude, $A_{x}=\sqrt{\beta_{x} J_{x}}$, has a maximum betatron value $\beta_{x}$. The maximum divergence at this point is $A_{x}^{\prime}=\sqrt{J_{x} / \beta_{x}}$. In this case we can write $A_{x}^{\prime}=\frac{A_{x}}{\beta_{x}}=\frac{p_{x}}{p}$ [11]. In the extreme case when the transverse momentum $p_{x}$ is completely transferred to the longitudinal plane, it is boosted by $\gamma$, and we can write the change in momentum as

$$
\Delta p=\gamma p_{x}=\gamma \frac{p A_{x}}{\beta_{x}}
$$

With IOTA parameters in mind, a transverse Gaussian emittance of $\sigma_{x}=1 \times 10^{-6}$ $\mathrm{m}, \beta_{x}=5 \mathrm{~m}$, and $\gamma=300$ give a momentum deviation of $\approx 1 \%$, which is the same order of magnitude as the RF energy acceptance. Calculating the lifetime requires finding the total loss rate by integrating all space and momentum coordinates in the collision cross section that leads to particle loss.

2.5.2 Approximation of the Growth Rate Due to IBS. For IBS calculations in IOTA, we will quote the formulation derived in [12]. In this simplified treatment of IBS, a beam with a "pancake" distribution, where $\frac{\Delta p / p}{\gamma}<<\theta_{\perp}$, is considered, such that the longitudinal velocity spread is much smaller than the transverse spread in the beam frame. For a pancake Gaussian distribution, the growth rate for the RMS 
longitudinal momentum is [12]

$$
\frac{d}{d t}\left(\delta_{R M S}^{2}\right)=\frac{e^{4}}{4 \sqrt{2} m^{2} c^{3} \gamma^{3} \beta^{3}}\left\langle\frac{N \Psi\left(\theta_{x}, \theta_{y}, 0\right) L_{c}}{\sigma_{x} \sigma_{y} \sigma_{s} \sqrt{\theta_{x}^{2}+\theta_{y}^{2}}}\right\rangle_{s},
$$

where $N$ is the particle density, $e$ and $m$ are the particle charge and mass, $c$ is the speed of light, $\gamma$ and $\beta$ are the Lorentz factor and the ratio of the velocity to the speed of light, and $L_{c}$ is the Coulomb logarithm - the factor by which small angle scattering dominates over large angle scattering. The beam sizes $\sigma_{x, y}$ are given by Eq. 2.26, $\sigma_{s}$ is the equilibrium emittance given by SR damping and $\theta_{x, y}$ are the angular spreads. The bracket here denotes averaging these values around the ring. The pancake distribution function is given by

$$
\Psi\left(\theta_{x}, \theta_{y}, 0\right)=1+\frac{\sqrt{2}}{\pi} \ln \left(\frac{\theta_{x}^{2}+\theta_{y}^{2}}{2 \theta_{x} \theta_{y}}\right)-0.055\left(\frac{\theta_{x}^{2}-\theta_{y}^{2}}{\theta_{x}^{2}+\theta_{y}^{2}}\right) .
$$

For storage rings working above transition, the transverse growth rates are dominated by the energy deviations in locations of high dispersion. The emittance growth rates in both transverse planes are [12]

$$
\frac{d \epsilon}{d t}=\left\langle\mathcal{H} \frac{d}{d t}\left(\delta_{R M S}^{2}\right)\right\rangle
$$

where $\mathcal{H}$ is the dispersion invariant given in Eq. 2.56.

The OSC lattice configuration is designed to have a small emittance, and as such, the synchrotron damping is eclipsed by IBS. To overcome this obstacle, the number of electrons per bunch must be reduced until the growth rate due to IBS is below that of the synchrotron damping rate in the ring.

\subsection{Chromaticity}

Most circular storage rings operate within a very small tune spread, $\Delta Q$. The tune is calculated in the same manner as the phase advance - as seen in Eq. 2.20-but is integrated around the circumference of the ring, $R$, and is given in units of $2 \pi$ :

$$
Q(s)=\frac{1}{2 \pi} \int_{R} \frac{d s}{\beta(s)} .
$$


To have a sufficient dynamic aperture in a storage ring such as IOTA, chromaticity must be corrected with sextupoles. This is the equivalent of correcting the chromatic aberration in a lens. The tune spread is roughly estimated in terms of the chromaticity, $C$, and the momentum spread $\Delta p$ :

$$
\Delta Q \approx C \frac{\Delta p}{p}
$$

The momentum deviation causes the particle to be under- or over-focused by the quadrupoles. Supposing the focusing error is due to the energy deviation of the particle, we can calculate $C$ directly by integrating over the beta function $(\beta(s))$ through each focusing error $(\delta k)$ :

$$
\Delta Q \approx \frac{1}{4 \pi} \int \beta(s) \delta k(s) d s \approx\left[\frac{-1}{4 \pi} \int \beta(s) k(s) d s\right] \frac{\Delta p}{p} .
$$

Comparing this with the previous equation, we see that the chromaticity $C$ is simply the quantity in the square brackets. For the machine there are two $Q$ values that relate to horizontal and vertical oscillations separately. When designing the linear lattice of IOTA, it is important to keep the chromaticity of the ring low and leave enough physical space in the ring to add correcting sextupoles.

\subsection{2-D, 4-D and 6-D Beam Matching}

In order for a particle to be properly transported along a beam line or around a ring, it must have the appropriate 6 dimensional vector coordinates. We will first consider the simplest case: matching the beam in two dimensions in $x$ and $x^{\prime}$, following the theory outlined in $[13,14,15]$. Statistical beam parameters can be used to describe the RMS emittance of a 2-D ellipse; these parameters are called the second moments [13]. These second moments can be used to find the Twiss parameters and vice versa:

$$
\sigma_{\text {beam }}^{2 D}=\left(\begin{array}{cc}
\left\langle x^{2}\right\rangle-\langle x\rangle^{2} & \left\langle x x^{\prime}\right\rangle-\langle x\rangle\left\langle x^{\prime}\right\rangle \\
\left\langle x^{\prime} x\right\rangle-\left\langle x^{\prime}\right\rangle\langle x\rangle & \left\langle x^{\prime 2}\right\rangle-\left\langle x^{\prime}\right\rangle^{2}
\end{array}\right)=\epsilon_{x}\left(\begin{array}{cc}
\beta_{x} & -\alpha_{x} \\
-\alpha_{x} & \gamma_{x}
\end{array}\right) .
$$


If the horizontal Twiss functions $\left(\beta_{x}, \alpha_{x}\right)$ are known, a 2-D beam with the appropriate $x$ - $x^{\prime}$ coordinates can be generated. However, when considering a 4-D beam that is horizontally and vertically coupled, another method of constructing a covariance matrix must be formulated. If we consider the motion of a single particle in a lattice with elements which couple the motion horizontally and vertically, we expect there to be two distinct oscillation modes which we will denote by ' $I$ ' and ' $I I$ ' instead of ' $x$ ' and ' $y$ '. These motions are no longer purely horizontal or vertical, and confine the motion to the surface of a hyper-ellipse. In the Ripken notation [13], the particle on the hyper-ellipse (in $4 \mathrm{D}$ phase space) at a given position, $s$, can be described by the generating vector $\vec{z}(s)=\left(x, x^{\prime}, y, y^{\prime}\right)^{T}$. The vector $\vec{z}(s)$ can then be expressed in terms of two amplitudes and two phases:

$$
\vec{z}(s)=\sqrt{\epsilon_{I}}\left[\vec{z}_{1}(0) \cos \phi_{I}-\vec{z}_{2}(0) \sin \phi_{I}\right]+\sqrt{\epsilon_{I I}}\left[\vec{z}_{3}(0) \cos \phi_{I I}-\vec{z}_{4}(0) \sin \phi_{I I}\right]
$$

where $\epsilon_{I, I I}$ and $\phi_{I, I I}$ are constants for the eigenmodes $I$ and $I I$. The column-vector $\vec{z}_{j}$ has the four components $\vec{z}_{j}=\left(V_{1, j}, V_{2, j}, V_{3, j}, V_{4, j}\right)^{T}$ which are related to the Ripken parameters with the following matrix equation:

$$
\left|V_{i, j}\right|=\left(\begin{array}{cccc}
\sqrt{\beta_{x I}} \cos \Phi_{x I} & \sqrt{\beta_{x I}} \sin \Phi_{x I} & \sqrt{\beta_{x I I}} \cos \Phi_{x I I} & \sqrt{\beta_{x I I}} \sin \Phi_{x I I} \\
\sqrt{\gamma_{x I}} \cos \Phi_{x I} & \sqrt{\gamma_{x I}} \sin \Phi_{x I} & \sqrt{\gamma_{x I I}} \cos \Phi_{x I I} & \sqrt{\gamma_{x I I}} \sin \Phi_{x I I} \\
\sqrt{\beta_{y I}} \cos \Phi_{y I} & \sqrt{\beta_{y I}} \sin \Phi_{y I} & \sqrt{\beta_{y I I}} \cos \Phi_{y I I} & \sqrt{\beta_{y I I}} \sin \Phi_{y I I} \\
\sqrt{\gamma_{y I}} \cos \Phi_{y I} & \sqrt{\gamma_{y I}} \sin \Phi_{y I} & \sqrt{\gamma_{y I I}} \cos \Phi_{y I I} & \sqrt{\gamma_{y I I}} \sin \Phi_{y I I}
\end{array}\right)
$$

where the envelope functions $\sqrt{\beta_{I, I I}}$, angle envelopes $\sqrt{\gamma_{I, I I}}$, and phase functions $\cos \phi_{I, I I}$ and $\sin \phi_{I, I I}$ are shown in Figure 2.4. One can get the particle coordinates expressed in terms of Ripken parameters by substituting Eq. 2.66 into 2.65:

$$
\vec{z}=\left(\begin{array}{c}
\sqrt{\epsilon_{I} \beta_{x I}} \cos \left(\Phi_{x I}+\phi_{I}\right)+\sqrt{\epsilon_{I I} \beta_{x I I}} \cos \left(\Phi_{x I I}+\phi_{I I}\right) \\
\sqrt{\epsilon_{I} \gamma_{x I}} \cos \left(\Phi_{x I}+\phi_{I}\right)+\sqrt{\epsilon_{I I} \gamma_{x I I}} \cos \left(\Phi_{x I I}+\phi_{I I}\right) \\
\sqrt{\epsilon_{I} \beta_{y I}} \cos \left(\Phi_{y I}+\phi_{I}\right)+\sqrt{\epsilon_{I I} \beta_{y I I}} \cos \left(\Phi_{y I I}+\phi_{I I}\right) \\
\sqrt{\epsilon_{I} \gamma_{y I}} \cos \left(\Phi_{y I}+\phi_{I}\right)+\sqrt{\epsilon_{I I} \gamma_{y I I}} \cos \left(\Phi_{y I I}+\phi_{I I}\right)
\end{array}\right) .
$$




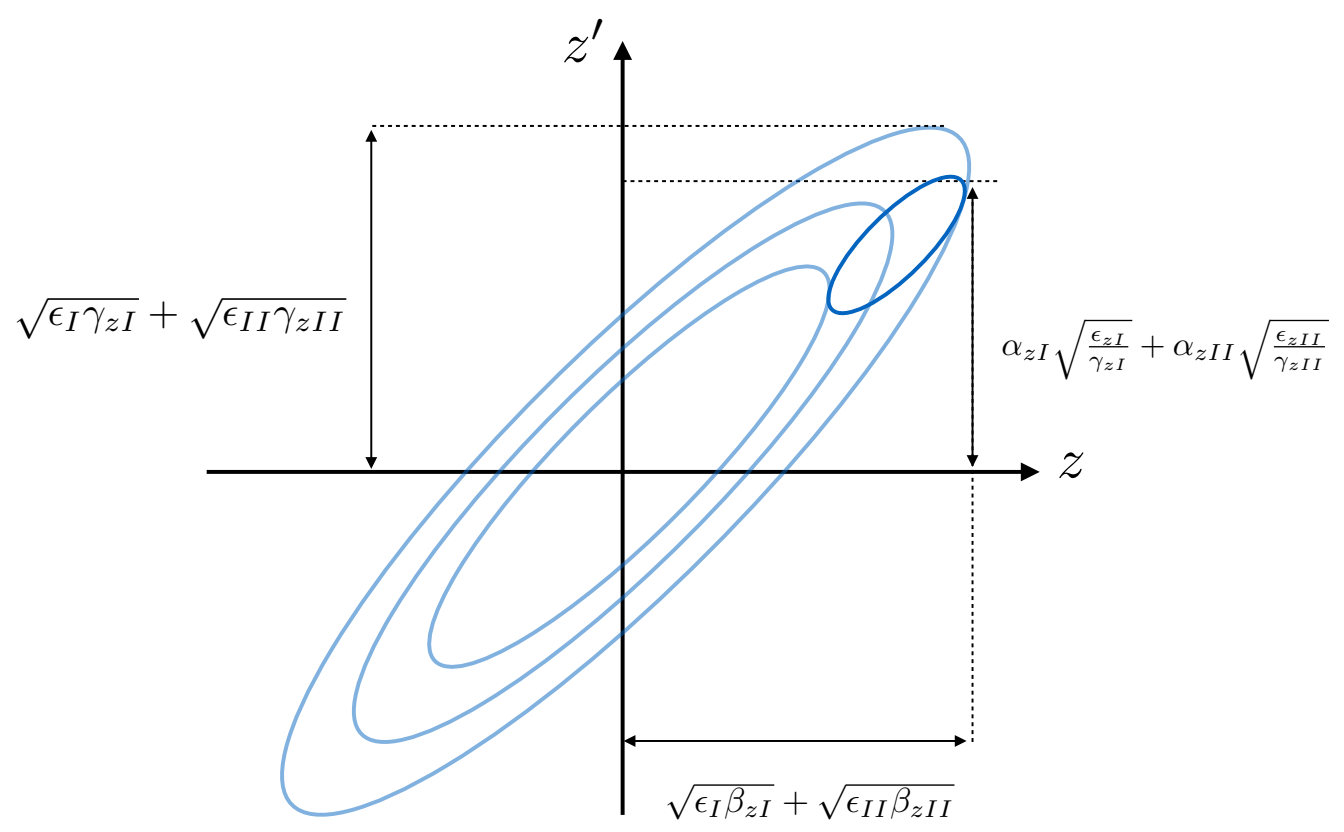

Figure 2.4. On each consecutive turn around a storage ring, the phase space position traces out a helical path on the two-dimensional surface of a torus in the fourdimensional $x-x^{\prime}$ and $y-y^{\prime}$ planes.

For a circular machine, the lattice functions are periodic, and the generating vectors $\vec{z}_{1, \ldots, 4}$ differ from their initial values by only a phase factor $\left(2 \pi Q_{I, I I}\right)$ which accounts for a transformation once around the machine:

$$
\begin{aligned}
\vec{z}(s)=\sqrt{\epsilon_{I}}\left[\vec{z}_{1}(0) \cos \phi_{I}+2 \pi Q_{I}-\vec{z}_{2}(0) \sin \phi_{I}+2 \pi Q_{I}\right] \\
+\sqrt{\epsilon_{I I}}\left[\vec{z}_{3}(0) \cos \phi_{I I}+2 \pi Q_{I I}-\vec{z}_{4}(0) \sin \phi_{I I}+2 \pi Q_{I I}\right] .
\end{aligned}
$$

We can extend the "normal form" technique of the 4D case outlined above to 6 dimensions. The new eigenvectors make up a $6 \times 6$ matrix; the beam distribution 
is generated using the following matrix multiplication:

$$
\sigma_{\text {beam }}^{6 D}=V_{6 \times 6} \epsilon_{6 \times 6} V_{6 \times 6}^{T}=\left(\begin{array}{cccccc}
\left\langle x^{2}\right\rangle & \left\langle x x^{\prime}\right\rangle & \langle x y\rangle & \left\langle x y^{\prime}\right\rangle & \langle x l\rangle & \langle x \delta\rangle \\
\left\langle x^{\prime} x\right\rangle & \left\langle x^{\prime 2}\right\rangle & \left\langle x^{\prime} y\right\rangle & \left\langle x^{\prime} y^{\prime}\right\rangle & \left\langle x^{\prime} l\right\rangle & \left\langle x^{\prime} \delta\right\rangle \\
\langle y x\rangle & \left\langle y x^{\prime}\right\rangle & \left\langle y^{2}\right\rangle & \left\langle y y^{\prime}\right\rangle & \langle y l\rangle & \langle y \delta\rangle \\
\left\langle y^{\prime} x\right\rangle & \left\langle y^{\prime} x^{\prime}\right\rangle & \left\langle y^{\prime} y\right\rangle & \left\langle y^{\prime 2}\right\rangle & \left\langle y^{\prime} l\right\rangle & \left\langle y^{\prime} \delta\right\rangle \\
\langle l x\rangle & \left\langle l x^{\prime}\right\rangle & \langle l y\rangle & \left\langle l y^{\prime}\right\rangle & \left\langle l^{2}\right\rangle & \langle l \delta\rangle \\
\langle\delta x\rangle & \left\langle\delta x^{\prime}\right\rangle & \langle\delta y\rangle & \left\langle\delta y^{\prime}\right\rangle & \langle\delta l\rangle & \left\langle\delta^{2}\right\rangle
\end{array}\right)
$$

where $\epsilon_{6 \times 6}$ is the diagonal matrix of emittances $\epsilon_{x}, \epsilon_{y}$, and $\epsilon_{z}$.

In tracking particles through the OSC bypass, it is necessary to use a properly matched $6 D$ beam. Appendix $\mathrm{C}$ contains the MadX script used to generate the beam distributions from the eigenvalues of the IOTA ring in the OSC configuration. 


\section{CHAPTER 3}

\section{NONLINEAR INTEGRABLE OPTICS}

\subsection{Theory of Nonlinear Integrable Optics}

A system is said to be integrable if one is able to predict analytically the motion of a particle in the system given the initial conditions. To do this, the system must have as many constants of motion as degrees of freedom under some canonical transformation. The problem of solving non-integrable Hamiltonian systems was first encountered in the attempt to describe planetary motion around the sun. If there is only a single planet, the system is integrable, but if additional planets are introduced, no exact solution for the motion of the planets exists. However, one can attempt to solve for the motion by first preserving the system's integrability considering only the planets' interactions with the sun, and then by systematically including the interactions between planets in a perturbative fashion. In this way, one can develop series expansions for the solutions of these equations. In such a system it is not possible to prove whether the orbits are stable as $t$ goes to infinity. This can be understood if we consider two planets with equal or approximately equal periods. In this case, the motion of the system can be driven into resonance by their periodic interaction, and subsequent instability can occur.

An attempt to understand stability over a long period was put forward by Kolmogorov, Arnold, and Moser (KAM theory). In Section 2.1 we develop the particle motion in a storage ring using both Twiss parameters and characteristic rays. We will now depart from this formalism and consider the Hamiltonian for such a system. For particles tracing out an ellipse in phase space as seen in Figure 2.2, they do so along a curve known as the action I which is an integral of motion. ${ }^{3}$ Each point on a phase

${ }^{3}$ Generally speaking, single particles have actions, while beam distributions have emittances - an invariant volume in phase space. 
curve will now be labeled by a single valued function $\theta$. In general, the Hamiltonian $\mathcal{H}$ is a function of the two canonical coordinates $I$ and $\theta$. KAM theory states that for such a non-integrable system, the Hamiltonian can be decomposed into two terms: an integrable term $H_{0}$, and a non-integrable, perturbative one $\epsilon V$ :

$$
\mathcal{H}(I, \theta)=H_{0}(I)+\epsilon V(I, \theta)
$$

where some of the periodic orbits survive if $\epsilon<<1$. Since $H_{0}$ is dependent only on $I$, it is time-independent and a constant of the motion. The second term introduces a perturbation that depends on the phase of the unperturbed motion, and therefore changes the unperturbed action $I$. KAM theory specifies quantitatively what maximum level of perturbation can be applied if stability is to be preserved. In the case of stable motion, the actions remain constant, and the angle variables increase only linearly with time; in these cases, errors introduced into the system will not result in resonance. An important consequence of KAM theory is that for a large set of initial conditions the motion remains perpetually quasi-periodic, i.e., the motion in phase space never closes. In an accelerator ring, this corresponds to a tune that is not on a resonance.

A recent solution found by Danilov and Nagaitsev [16] for a nonlinear integrable accelerator using special magnets has been studied and will be implemented in IOTA. A desired spread of frequencies can be achieved by adding a nonlinear potential to the Hamiltonian. To create this potential, we start with a strong focusing lattice (containing quadrupoles) in the horizontal and vertical planes and add a time-dependent ${ }^{4}$ potential, $V$. Such a lattice has the following Hamiltonian:

$$
\mathcal{H}=\frac{p_{x}^{2}+p_{y}^{2}}{2}+\frac{g_{x}(s) x^{2}+g_{y}(s) y^{2}}{2}+V(x, y, s)
$$

${ }^{4}$ In an accelerator, the components are located along defined positions on the reference trajectory. The time, $t$, at which the particle arrives at a component is not always known; therefore it is more convenient to use the path length, $s$, as the independent value rather than $t$. 
a.

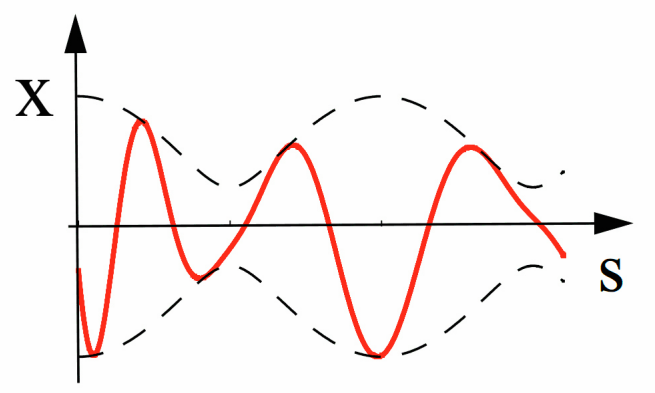

C.

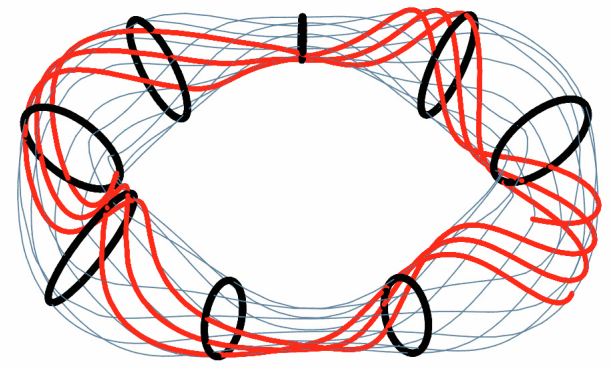

b.

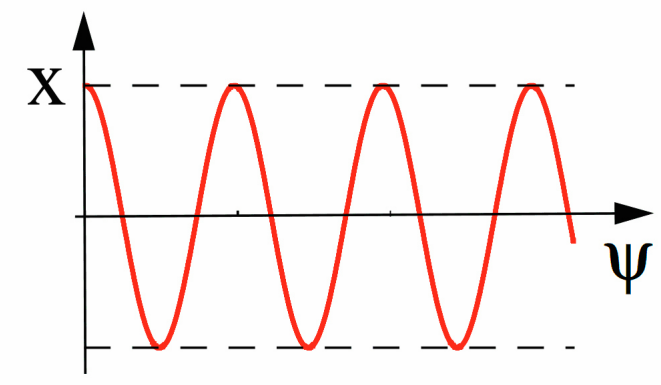

d.

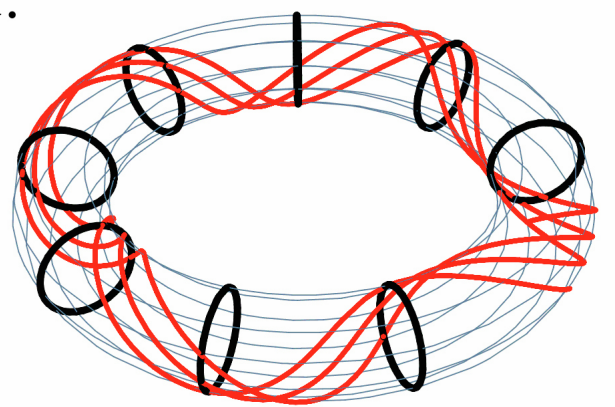

Figure 3.1. Particle trajectories (a.,b.) and phase-space ellipses (c.,d.) in the original and normalized variables.

where the phase space coordinates $x$ and $y$ are the transverse positions, $p_{x}$ and $p_{y}$ the momenta, and $g_{x}(s)$ and $g_{y}(s)$ the focusing gradients in the quadrupoles. Since a lattice can have its gradients scaled to accommodate any arbitrarily massive particle, the mass typically found in the Hamiltonian is omitted. This Hamiltonian resembles a harmonic oscillator with an added time-dependent potential.

If we consider a lattice which is axially symmetric in focusing, i.e., $g_{x}(s)=$ $g_{y}(s)$, the betatron phase advance $\psi(s)$ can be chosen as a new independent "time" variable. We then arrive at a new Hamiltonian, $\tilde{\mathcal{H}}$, with the same physical motion as $\mathcal{H}:$

$$
\frac{\partial \tilde{\mathcal{H}}}{\partial p_{q}}=\frac{1}{\lambda(\psi(s))} \frac{\partial \mathcal{H}}{\partial p_{q}} ; \quad \frac{\partial \tilde{\mathcal{H}}}{\partial q}=\frac{1}{\lambda(\psi(s))} \frac{\partial \mathcal{H}}{\partial q},
$$

where the subscript $q$ denotes $x$ or $y$, and $\lambda(\psi(s))$ is some function of the new "time." Under normalized-variable substitution (denoted by ${ }_{N}$ ), the "time" dependence can 
be moved into a non-linear potential term, which results in a new potential:

$$
\tilde{V}\left[x_{N}, y_{N} ; \psi\right]=\beta[s(\psi)] V\left[x_{N} \sqrt{\beta(\psi)}, y_{N} \sqrt{\beta(\psi)} ; s(\psi)\right] .
$$

This normalization via the beta function results in a time-independent Hamiltonian with the final form

$$
\tilde{\mathcal{H}}_{N}=\frac{p_{x N}^{2}+p_{y N}^{2}}{2}+\frac{x_{N}^{2}+y_{N}^{2}}{2}+\tilde{V}\left(x_{N}, y_{N}\right) .
$$

The new potential is chosen to possess a second integral of motion, which can be guaranteed by the choice of new generalized coordinates where position and momentum variables can be separated, making the system exactly integrable and realizable by a magnetic potential. As shown in [16] and [17], the integrable potential is obtained with elliptic coordinates. The potential contains two repulsive singularities along the horizontal separated by a distance $2 c$ as shown in Figure 3.2.

The betatron functions $\beta^{*}(\Delta Q, L)$ at the longitudinal center of the $1.8 \mathrm{~m}$ nonlinear magnets are a function of the phase advance inside the magnet, $\Delta Q$, and the length of the magnet, $L$. The amplitudes, $A^{*}$, at the same location were determined prior to the design of the IOTA lattice; they determine the allowable maximum betatron functions and amplitudes $\left(\beta_{x, y}^{\max }\right.$ and $\left.A_{x, y}^{\max }\right)$ in the ring, outside of the nonlinear inserts. Following from Eq. 2.25, and knowing that the emittance in the ring is invariant, $\beta_{x, y}^{\max }$ and $A_{x, y}^{\max }$ can be found with the following equation:

$$
\frac{A_{x, y}^{\max }}{\sqrt{\beta_{x, y}^{\max }}}=\frac{A_{x, y}^{*}}{\sqrt{\beta_{x, y}^{*}}} .
$$

For IOTA, the lattice that offers a potential such as the one outlined above contains two periodic parts:

1. a drift space with equal horizontal and vertical beta-functions, followed by

2. an optics insert (with overall focusing $k$ in each plane) containing quadrupoles and dipoles which has the transfer matrix of a thin axially symmetric lens. 


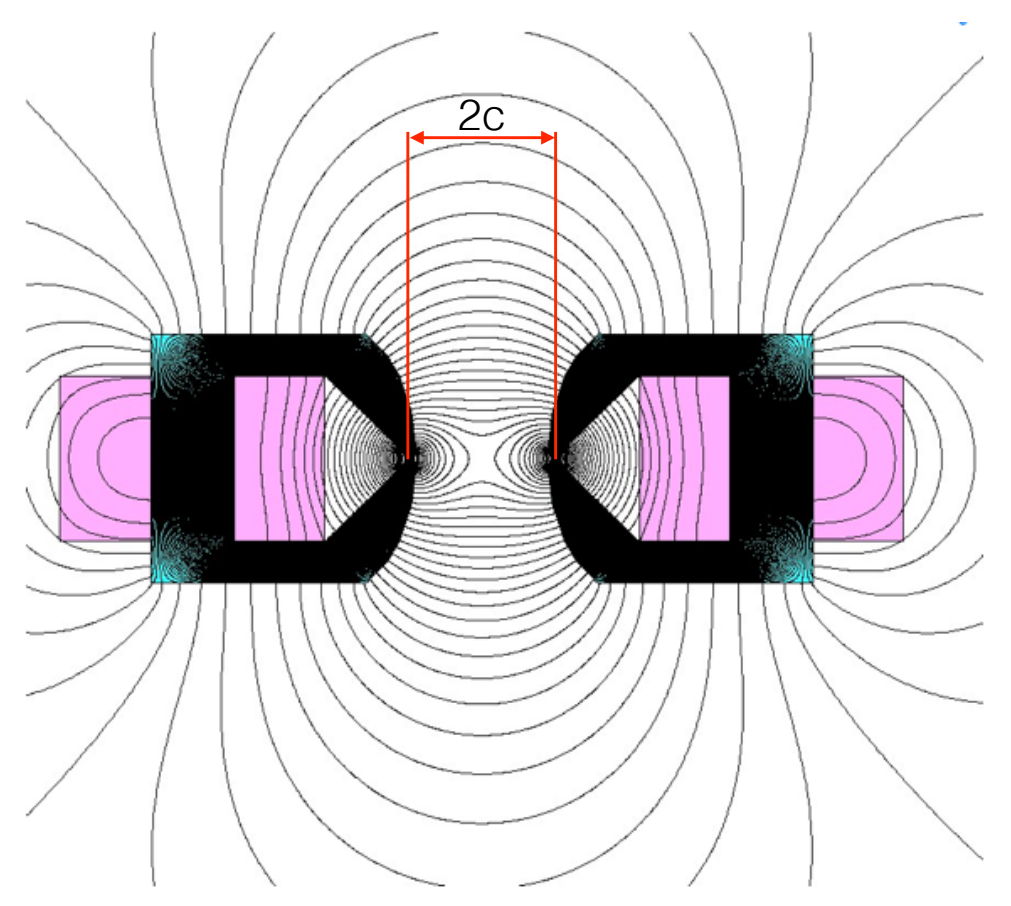

Figure 3.2. A transverse cross-section of the nonlinear magnet with field singularities located at $x= \pm c= \pm 1 \mathrm{~cm}$. The pink segments show the location of the excitation coils, and the black segments show the cross-section of a continuous return yoke. For a more more detailed description of these magnets, see Ref. [18]. 
A simplified picture of a lattice with nonlinear potentials is shown in Figure 3.3. The top picture emphasizes that the nonlinear potential is a correction to multiple "thin lenses"; the bottom picture is an expansion of the top showing that the "thin lens" can be any number of focusing magnets that flip the slopes of the horizontal and vertical beta functions.
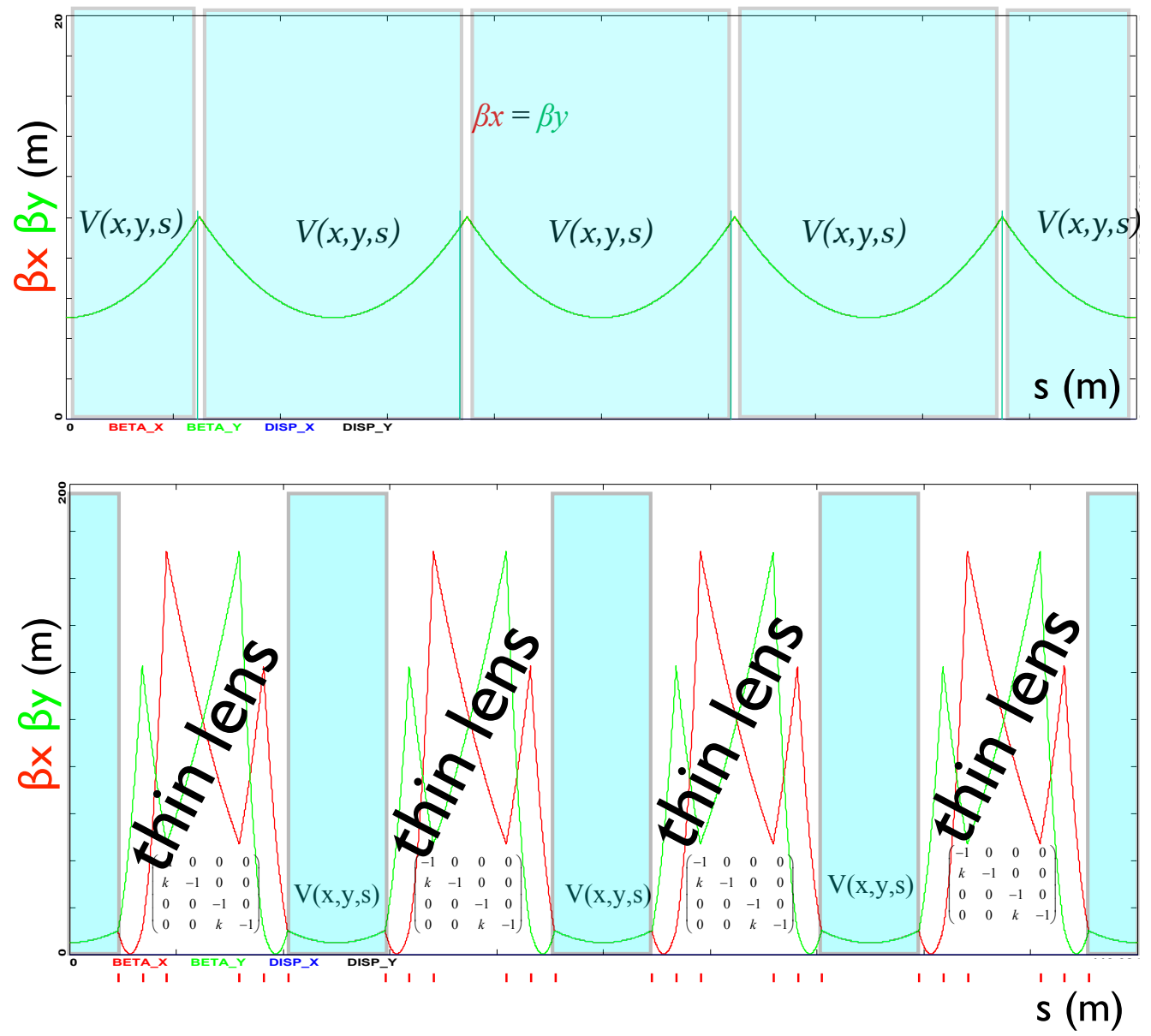

Figure 3.3. Horizontal and vertical beta functions ( $\beta_{x}$ in red and $\beta_{y}$ in green). The integrable optics sections (with nonlinear potential $V$ ) can be placed periodically. Note: this configuration has 4 integrable optics sections whereas the current design of IOTA will only have two. The matrix denotes equal focusing in the horizontal and vertical planes.

For IOTA, the nonlinear magnetic potential is described as a series expansion with the first and second terms resembling quadrupole and octupole terms. The form 
of the magnetic potential is as follows:

$$
\begin{aligned}
& \tilde{V}_{\text {magnetic }}(x, y) \approx \frac{x^{2}}{2}+\frac{y^{2}}{2} \\
&+t \cdot \Re\left((x+i y)^{2}+\frac{2}{3}(x+i y)^{4}+\frac{8}{15}(x+i y)^{6}+\frac{16}{35}(x+i y)^{8}+\ldots\right),
\end{aligned}
$$

where $t$ is the magnitude of the nonlinear potential. A cross-section of the nonlinear magnet along with the field lines is shown in Figure 3.2. For $t<0$, the potential provides additional focusing in $x$, and defocusing in $y$. The tune spread gained by the inclusion of these nonlinear lenses is given in terms of the strength of the nonlinear field. For particles with small amplitudes, the betatron frequencies are [16]:

$$
\nu_{1}=\nu_{0} \sqrt{1+2 t} \quad \nu_{2}=\nu_{0} \sqrt{1-2 t},
$$

where $\nu_{0}$ is the unperturbed linear-motion betatron frequency. For stable smallamplitude motion, we must have $-0.5<t<0$ to ensure that the frequency is positive and real in both planes. For large amplitudes, particles sample a weaker nonlinear field $t$, and the betatron frequencies in each plane of motion approach $\nu_{0}$. Therefore, a betatron frequency spread of $100 \%$ in one plane and $\approx 40 \%$ in the other is achieved.

Before running the one- and two-nonlinear integrable optics experiments, an intermediate option is being explored to truncate the full magnetic potential after the octupole term. This simplification of the nonlinear potential would be constructed by stacking octupole magnets in the drift space in order to replicate the first two terms in the potential. Simulations are currently being carried out to explore the feasibility of such a configuration.

The trajectories of particles in an integrable nonlinear lattice occupy large regions of stability in phase space, and have a large betatron frequency spread that allows the reduction of particle loss via Landau damping. 


\section{CHAPTER 4}

\section{LATTICE DESIGN FOR IOTA}

The IOTA ring is intended to be a cost-effective and flexible ring, allowing multiple experiments with the same physical setup, or with minimal movement of the components. The IOTA ring will be capable of operating with $50-150 \mathrm{MeV}$ electrons and later, with $2.5 \mathrm{MeV}$ protons. There are four different studies proposed with nonlinear lenses in IOTA: two with electron lenses and two with special nonlinear magnets. In all experiments, the circulating beam will be kicked in the transverse planes to "sample" nonlinearities, after which the phase space trajectories will be studied using beam position monitors (BPMs) and synchrotron light outputs. The OSC experiment is discussed at length in Chapter 5.

\subsection{Parameters for the Nonlinear Integrable Optics Experiments}

The IOTA ring sits downstream of the ASTA photoinjector and superconducting RF cryomodules. In Figure 4.1, the beam travels down the linac from the left and is diverted by two dipoles into the IOTA ring where it circulates clockwise.

There are several constraints that exist outside the parameters of the integrable optics experiments, some of which are determined by the linac upstream of the IOTA ring. These constraints include:

1. the total circumference of the ring, $\approx 40 \mathrm{~m}$, which is restricted by the dimensions of the experimental hall - the physical extent of the dipoles must be kept $5^{\prime} 11^{\prime \prime}$ from each of the hall walls to allow forklifts to move freely;

2. a minimum number of quadrupole magnets (30 of which are repurposed), and 8 dipole magnets (four $30^{\circ}$ magnets and four $60^{\circ}$ magnets);

3. BPM sensitivity of at least $2 \times 10^{9}$ electrons per bunch (at $>10^{10}$ particles per bunch, there are undesired collective effects); 


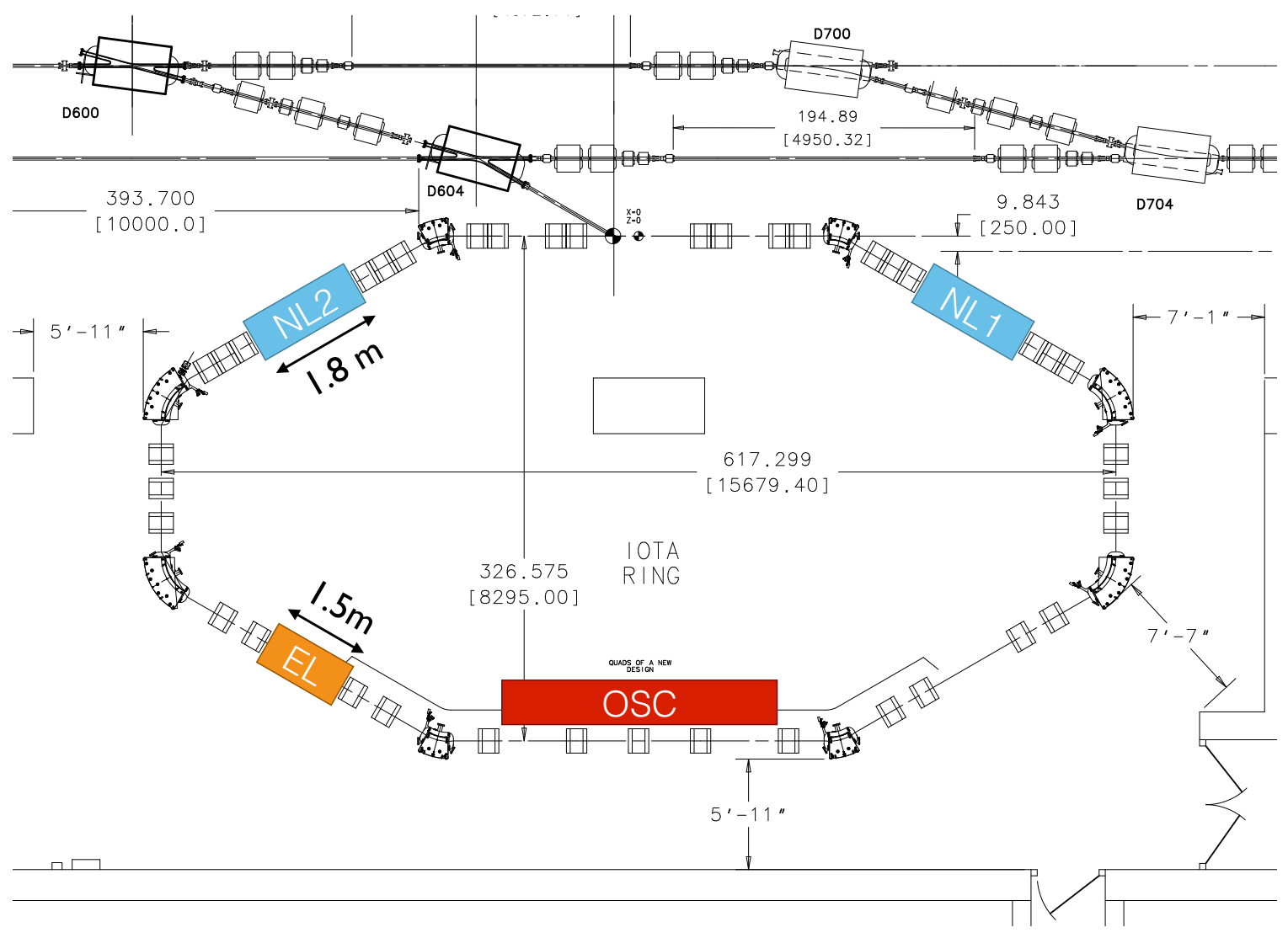

Figure 4.1. The IOTA ring layout showing eight dipoles, 39 quadrupoles and the location of the three experiments: nonlinear, OSC and electron lens. Dimensions with no units are in inches; millimeters in brackets.

4. a momentum spread $\frac{\Delta p}{p} \approx 10^{-4}$ upstream of the ASTA linac;

5. positive damping decrements in all planes for beam stability.

The requirements for the nonlinear integrable optics experiments are as follows (see Section 4.3):

1. accommodate three nonlinear magnet experiments: an experiment with only one nonlinear magnet, another with two nonlinear magnets, and a third one with four nonlinear magnets; each experiment satisfying the following conditions:

(a) nonlinear magnet(s) of length $1.8 \mathrm{~m}$,

(b) a betatron phase advance of 0.3 (in units of $2 \pi$ ) inside the magnets and a tune of $0.5 \times n$ between the magnets, where $n$ is an integer, and 
(c) a beta function not exceeding $12 \mathrm{~m}$ in the horizontal plane and $9 \mathrm{~m}$ in the vertical plane.

2. a $75 \mathrm{~cm}$ long solenoid to house an electron lens (see Section 4.4) with

(a) $\beta_{x}=\beta_{y}=3 \mathrm{~m}$ and

(b) a transfer matrix of identity (or integer tune) for the entire ring.

The only constraints relevant to the initial design of the ring set by the OSC experiment are:

1. two $\approx 5$ meter straight sections, one for beam injection and acceleration via an RF cavity, and the other for the OSC bypass, and

2. two-fold, mirror lattice symmetry set by the location of the OSC bypass and wigglers.

The last constraint listed above minimizes the number of circuits in the ring across all experiments. Since the optics in the OSC bypass must be symmetric around its center, i.e., the magnetic elements and their gradient strengths are identical for each half of the ring, the magnets can be paired and powered by the same power supply. We will keep this same pairing for all the experiments to eliminate the costs of re-wiring the magnets.

The nonlinear magnet experiments will need $\mathrm{a} \leq 1 \%$ control over the beta functions, and achieve a betatron phase to 0.001 accuracy. For this reason, quality of the dipole and quadrupole fields, optics stability, instrumentation systems and optics measurement techniques must be of current state-of-the-art [19, 20].

\subsection{Dipoles and Quadrupoles}

There are four $30^{\circ}$ and four $60^{\circ}$ sector dipoles, whose faces are perpendicular to the beam, in the IOTA ring. They were built for IOTA based on an earlier design of 

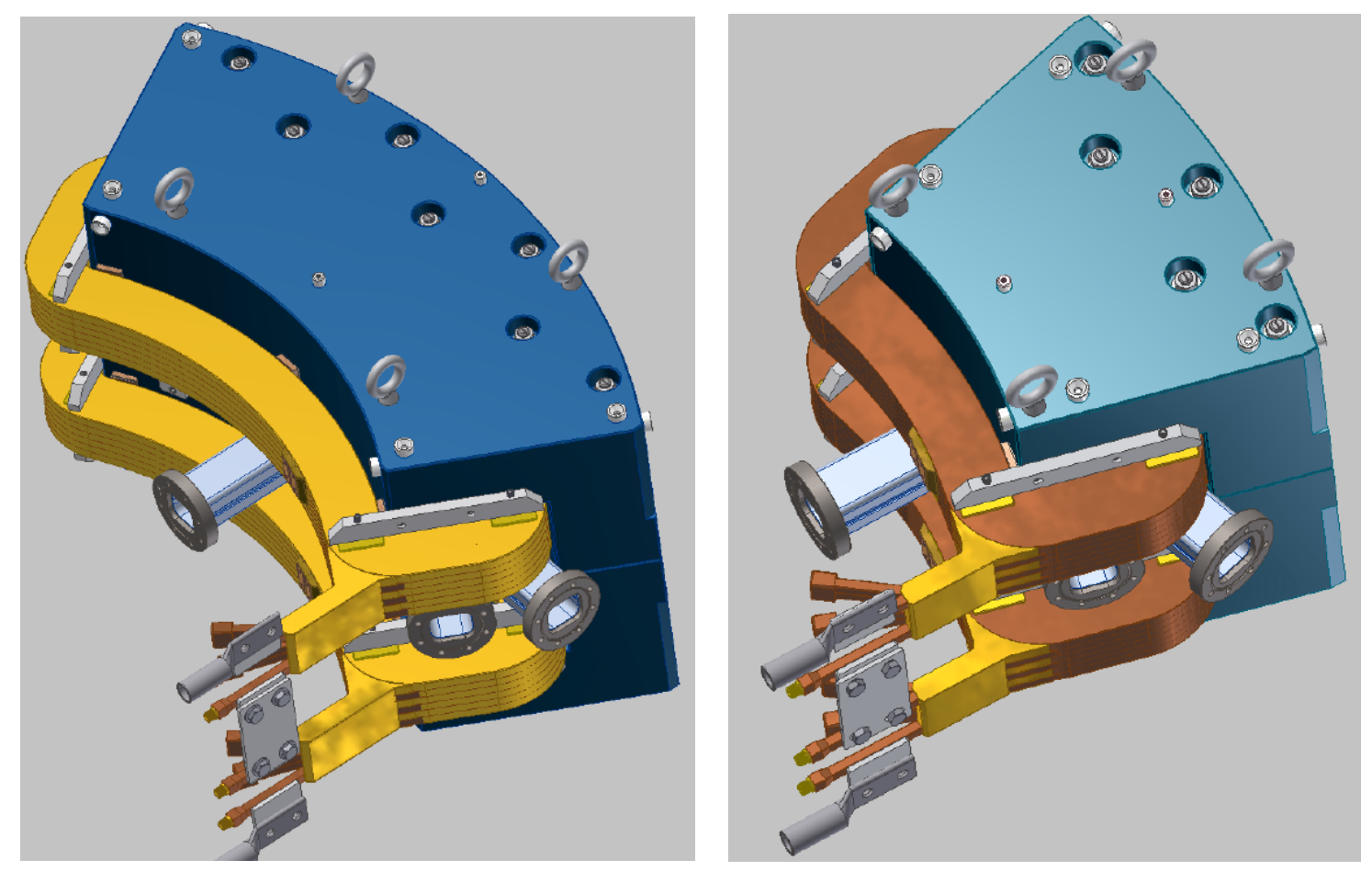

Figure 4.2. 3D drawings of the $60^{\circ}$ (left) and $30^{\circ}$ (right) dipoles for the IOTA ring. Ports for the beam and synchrotron light are included.

the ring which included 50 quadrupoles. They each have a bending radius of $0.7 \mathrm{~m}$, a magnetic field of 0.7 tesla and a good-field aperture of $48 \mathrm{~mm}$ in the horizontal plane. Figure 4.2 shows three dimensional CAD drawings of the two types of dipoles. All eight dipoles will be powered by a single power supply. The dipoles will be outfitted with pipes on either side (used for viewing synchrotron light) that extend tangentially from the center of the dipole. Ideally, quadrupoles would be placed up against the dipole coils for more efficient focusing and to reduce the overall circumference of the ring; however, the extent of these pipes prohibits the placement of directly adjacent quadrupoles. To accommodate the pipes, there must be $31 \mathrm{~cm}$ between the dipole and quadrupole coils. To conserve space, ports for vacuum pumping along with BPMs will also be placed in this space, the longitudinal extents of which will fit between the dipole's magnetic coils. Figure 4.3 shows the placement of the vacuum ports, BPMs and corrector packages between a $60^{\circ}$ dipole and an adjacent quadrupole. 


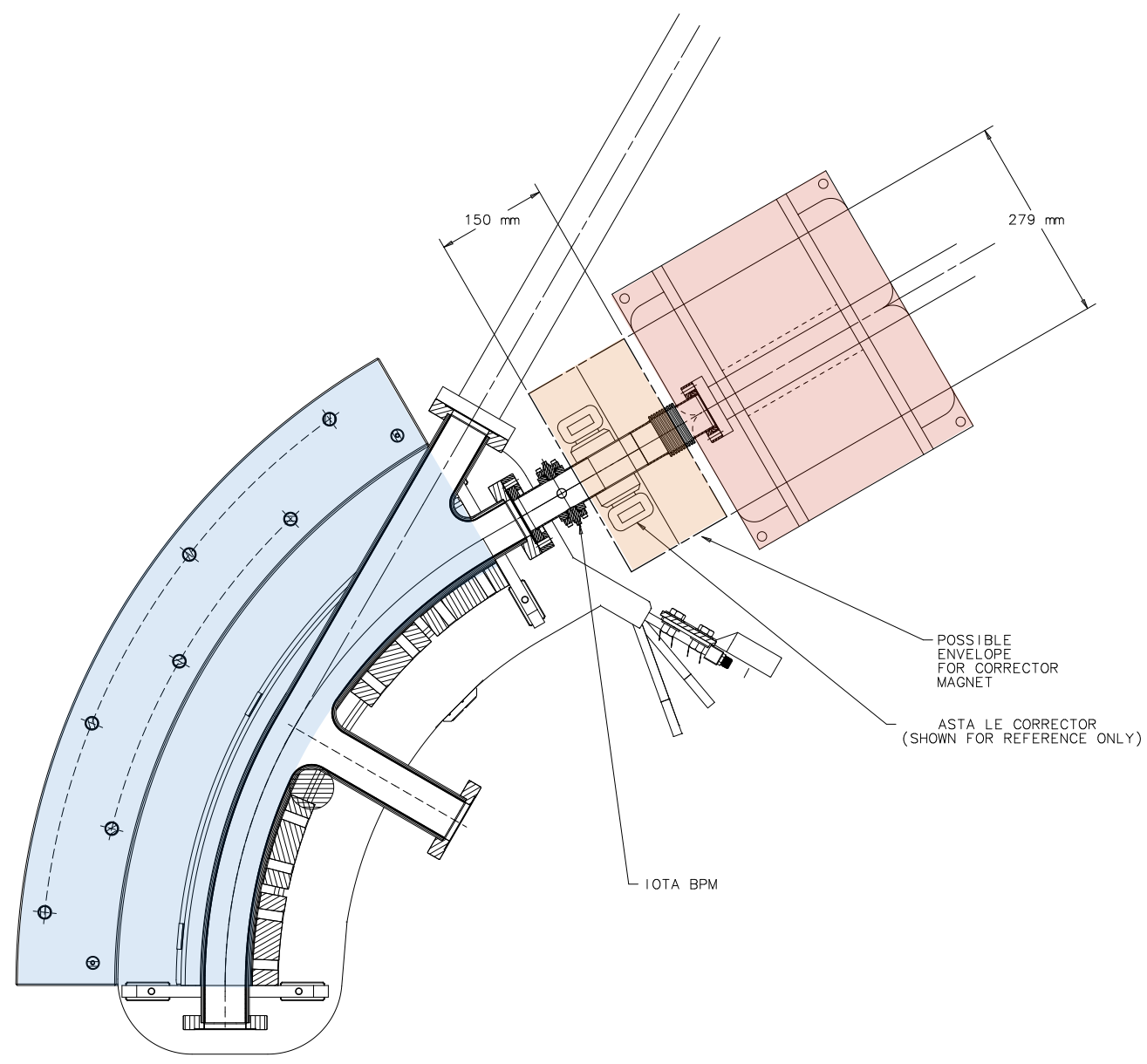

Figure 4.3. The synchrotron light port, extending tangentially from the center of the dipole (blue), prevents the quadrupole (red) from being placed up against the dipole coils. A corrector package and BPM (orange) will be placed in the space where the quadrupole would interfere with the synchrotron port, the BPM flanges extending into the quadrupole.

IOTA has received 32 water-cooled quadrupoles from the Joint Institute for Nuclear Research in Dubna. Each quadrupole has pole pieces that extend $21 \mathrm{~cm}$ along the beam line with coils that extend an additional $6.25 \mathrm{~cm}$ on either side as seen in Figure 4.4. The maximum achievable gradient and current are $19 \mathrm{~T} / \mathrm{m}$ and $300 \mathrm{~A}$ respectively. The quadrupole bore is $71 \mathrm{~mm}$. For lattice calculations and simulations, a hard edge model of the magnetic fields was used for both the quadrupoles and 

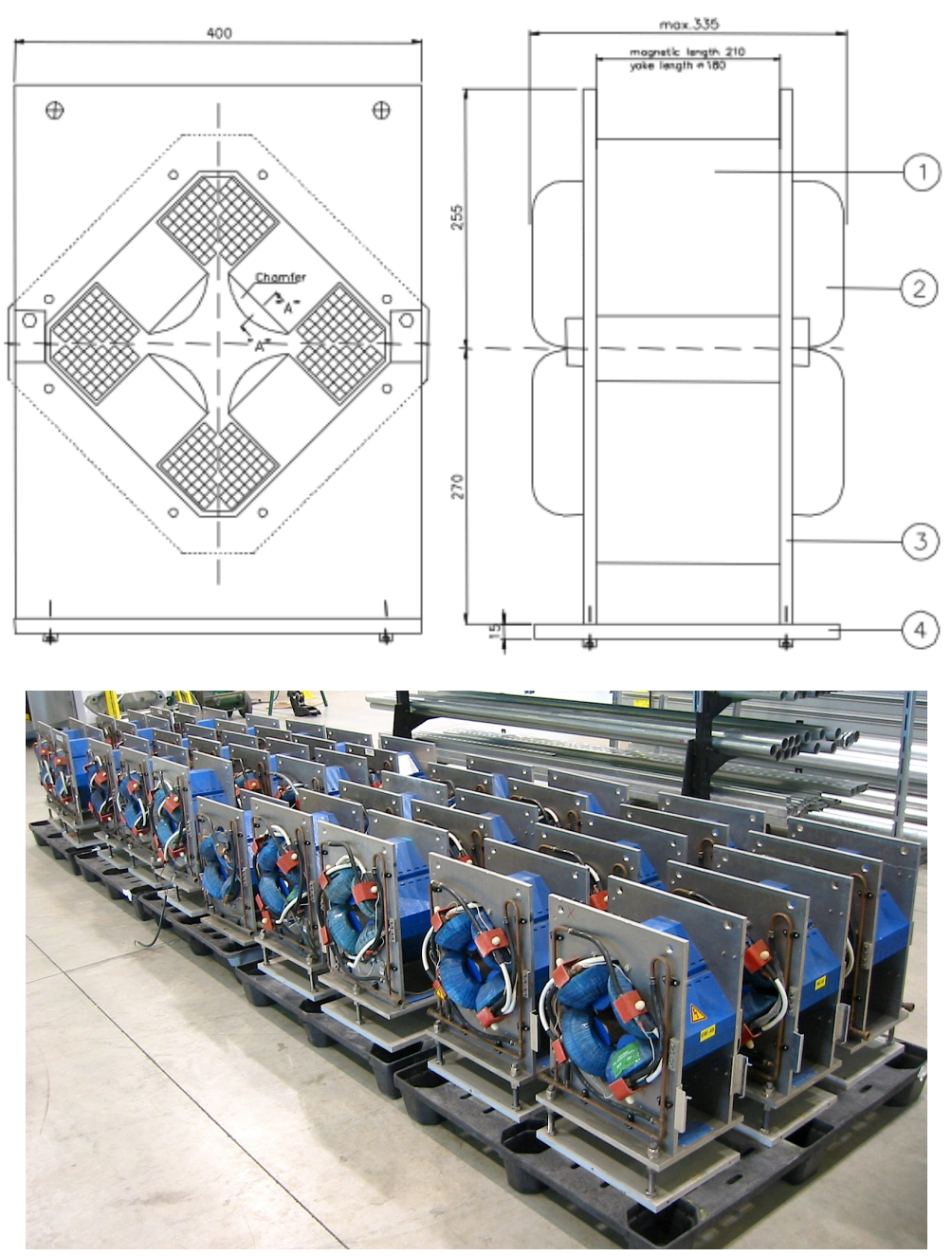

Figure 4.4. Top: schematic drawing of the Dubna quadrupole. Bottom: The quadrupoles delivered to Fermilab. At the time of this photograph, three had been set aside for testing and are not pictured here.

dipoles. An additional component for edge focusing was added on either side of each dipole to account for the field variation in the gap near the edge.

\subsection{Design of the Nonlinear Magnet Experiments}

The design process of the IOTA ring requires several iterations of different quadrupole configurations, and the dimensions of the ring shrink and expand with every iteration. Because iterating with the engineer takes some time, a Python script - in conjunction with an output of the MadX geometric coordinates of each 
element - was used to lay out the ring and extract its dimensions (see Appendix B). The design of the IOTA lattice was done in OptiM [21]. The OptiM file was converted to a MadX file using the built-in conversion tool in OptiM.

The integrable optics experiment with two nonlinear magnets was designed first because it has the most constraints. The beam's transverse profile must be round when entering and exiting the nonlinear magnet. This means that at these points, the beam must have equal horizontal and vertical beta functions (i.e., $\beta_{x}=\beta_{y} \equiv \beta$ ), and the dispersion and its derivative must be zero $\left(D_{x}=D_{x}^{\prime}=0\right)$.

The lattice must be "left-right" symmetric as indicated in Figure 4.1.5 Symmetric lattices have beta functions and dispersions whose derivatives are zero at the points of symmetry. In total there are five Twiss parameters that need to be controlled between the symmetry point and the beginning of the first nonlinear magnet, namely: $\alpha_{x, y}, \mu_{x, y}$, and $D_{x}^{\prime}$. Two additional "loose" constraints must be considered: the maximum amplitudes $\beta_{x, y}^{\max }$ allowed outside of the nonlinear magnets as described in Equation 3.6. One quadrupole is needed to match each constraint, and the "loose" constraint on the maximum amplitude limits the amount of space from one focusing element to the next; therefore a total of 14 quadrupoles - two triplets and four doublets - are needed between the two nonlinear magnets in order to preserve symmetry as seen in Figure 4.5. It is important to monitor the beam at the entrance and exit of each nonlinear magnet, therefore a $9 \mathrm{~cm}$ gap is placed on either side to accommodate BPMs.

It is natural to sandwich each nonlinear magnet between two triplets to preserve symmetry on either side of the magnet, and perhaps to make an expansion to a four-magnet configuration more feasible in the future. A triplet was added between

${ }^{5}$ This constraint is placed on the ring by the OSC experiment. 


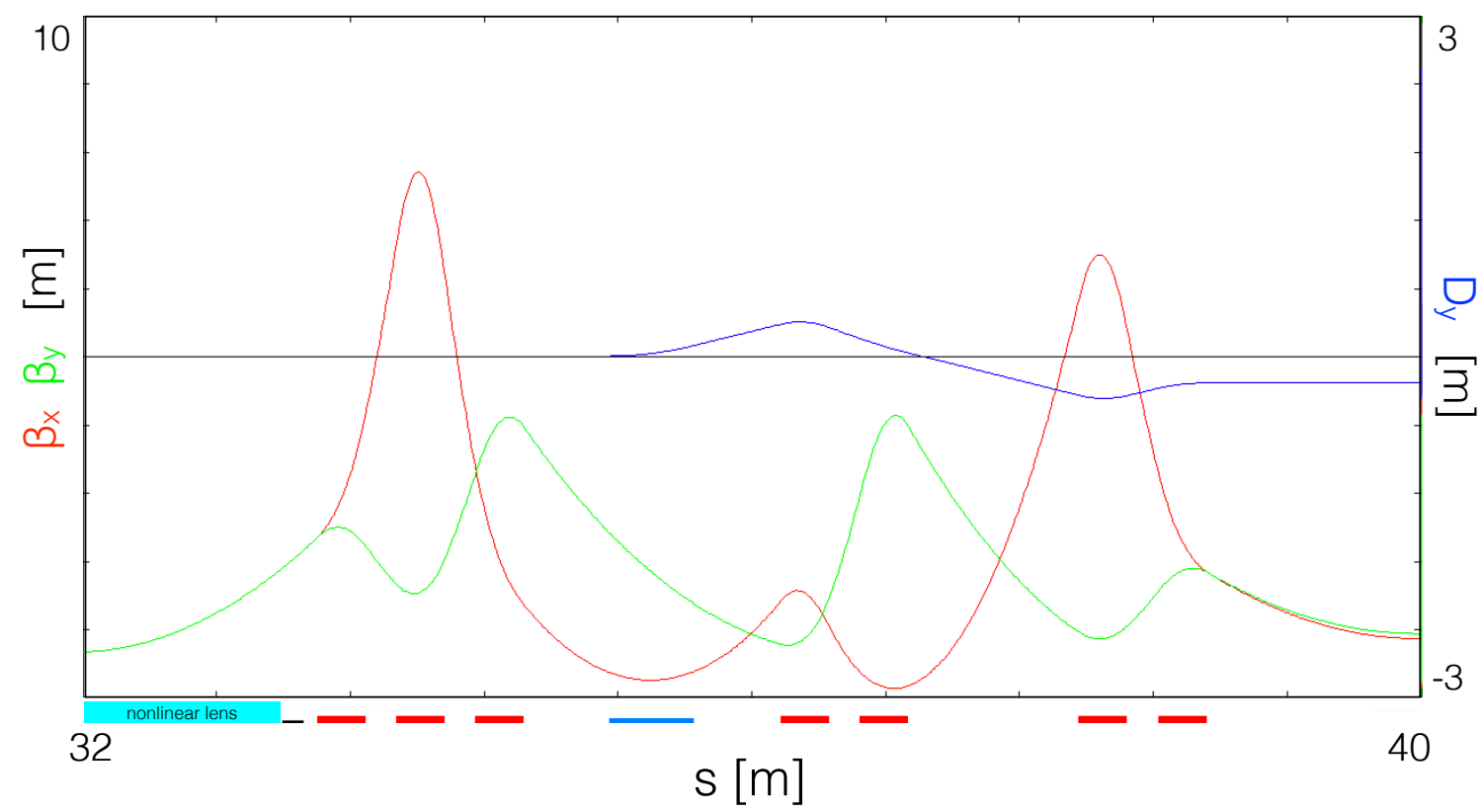

Figure 4.5. The horizontal and vertical beta functions originating in the middle of one nonlinear magnet and ending at the point of symmetry on the injection side of the ring. All slopes in the middle of the injection section must equal 0 to enforce symmetry. The quadrupoles appear as red rectangles, and the $30^{\circ}$ dipole, blue. The horizontal axis is labeled to be congruent with a plot starting and ending at the middle of the injection side of the ring.

the $60^{\circ}$ bends to better control the dispersion. Since the dispersion is high at the location of this triplet, space was left between the quadrupoles to allow for the addition of sextupoles for future chromatic corrections.

The additional 13 quadrupoles were placed with two main constraints: to minimize the beta function amplitudes and to preserve space for an electron lens in one of the remaining straight sections. Figure 4.6 shows the location of all the quadrupoles in the IOTA ring, and plots the beta functions and the horizontal dispersion functions for the entire ring. Figure 4.5 is a zoomed-in depiction of right-most side of Figure 4.6 . 


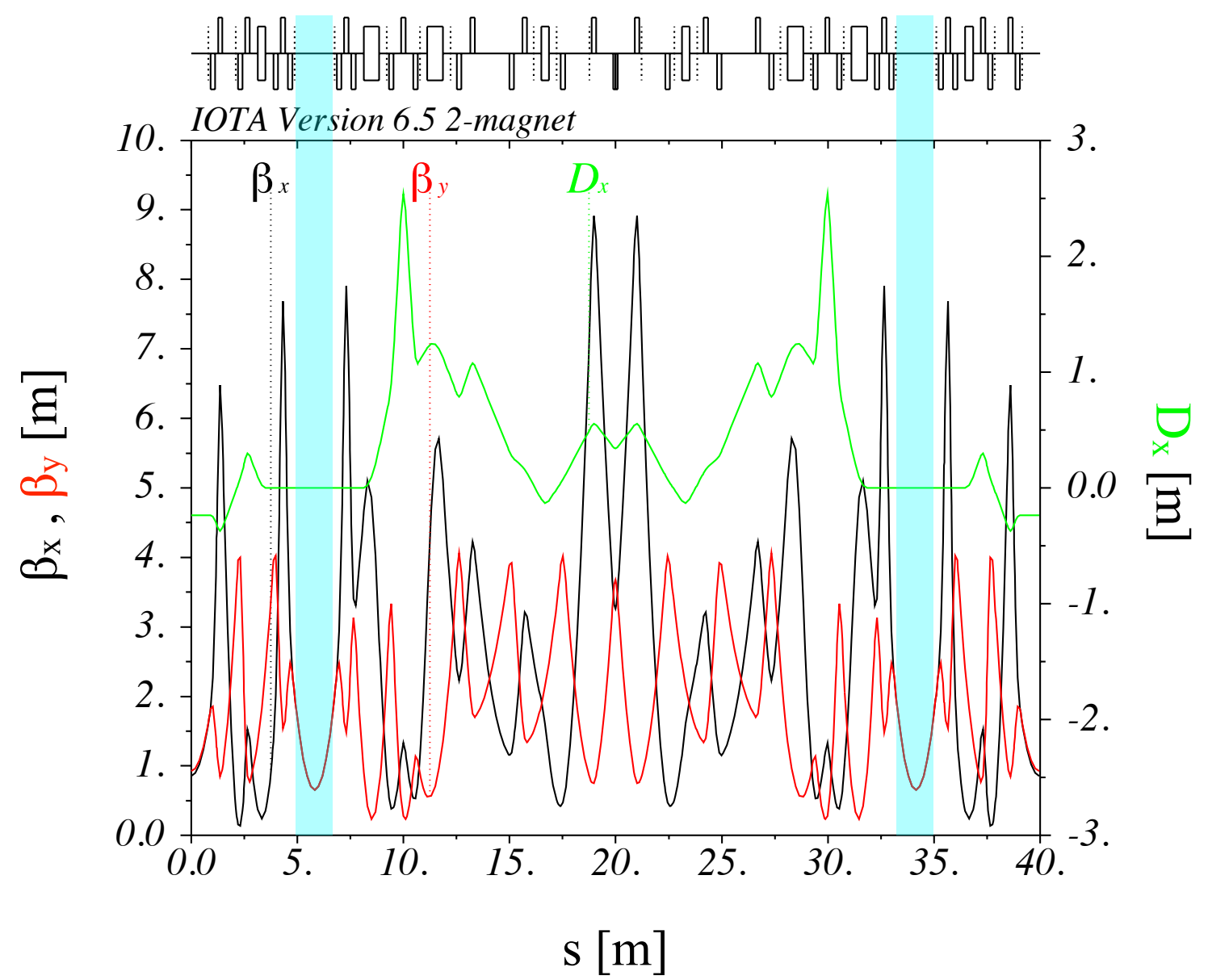

Figure 4.6. IOTA lattice with two nonlinear inserts. $\beta_{x}$ and $\beta_{y}$ are in black and red respectively; horizontal dispersion is in green. The nonlinear magnets are marked in cyan. The magnetic elements are represented by blocks above the plot: dipoles are centered on the horizontal; focusing and defocusing quadrupoles are smaller rectangles above and below the horizontal respectively; BPMs are marked with dotted lines. 
The magnet creating the nonlinear potential will be fitted into the drift space. The condition that this potential make the Hamiltonian time-independent requires that the magnetic field continuously change along the length of the nonlinear section. The betatron phase advance inside and outside the drift space in IOTA must be tightly controlled. The underlying linear lattice, when the nonlinear strength is set to zero, becomes a drift. It was found in previous simulations [16] that a corresponding drift with phase advance of 0.3 in both transverse directions was sufficient to produce a significant tune spread. Knowing the phase advance and the length of the drift, we can arrive at the appropriate vertical and horizontal beta functions at the beginning of the drift by using the following relation:

$$
\phi(s)=\int_{0}^{L / 2} \frac{d s}{\beta(s)}=\int_{0}^{L / 2} \frac{d s}{\beta^{*}+\frac{s^{2}}{\beta^{*}}}=\arctan \left(\frac{L}{2 \beta^{*}}\right),
$$

where $\phi(s)=0.3$ is the phase advance in the drift, $L=1.8 \mathrm{~m}$ is the length of the nonlinear insert, and $\beta^{*}$ is the waist of the beta function in the center of the drift where $\alpha^{*}=-\frac{1}{2} \frac{d \beta^{*}}{d s}=0$. The denominator in the second integrand describes the quadratic shape of the beta function in a drift. In general, it is easily seen that the phase advance in a drift must be $0<\phi(s)<0.5$. The phase advance throughout the ring for the two-nonlinear-magnet experiment is as follows:

1. a phase advance of $\mu_{x}=\mu_{y}=0.3$ in each nonlinear section;

2. between the nonlinear sections on the injection side of the ring, $\mu_{x}=0.5 n$ and $\mu_{y}=0.5 m$, where $n=2$ and $m=4 ;$

3. between the nonlinear sections on the "bottom half" of the ring, $\mu_{x}=0.5 n$ and $\mu_{y}=0.5 m$, where $n=7$ and $m=5$.

After obtaining a lattice solution for the two-nonlinear-lens experiment, the solution for the one-nonlinear-lens experiment was sought (see Table 4.3 for a list of parameters). IOTA will initially run with only one nonlinear magnet; if the experiment is deemed successful, a second one will be installed. The one- and two-nonlinear 
lens experiments have the same requirements; however, since in the one-nonlinearlens experiment only one straight section will contain a nonlinear magnet, it seems at first glance that the solution would require an asymmetric lattice. However, if we retain symmetry, the solution is quite simple: the first two conditions in the above list are kept the same, while the phase advances in the "bottom half" of the ring are increased from $\mu_{x}=3.5$ and $\mu_{y}=2.5$ to $\mu_{x}=3.7$ and $\mu_{y}=2.7$. Since one of the nonlinear sections will be without a magnet, it can simply be treated as a drift section. The phase advances between the nonlinear sections in the "bottom half" of the ring will be $\mu_{x}=3.7+0.3=0.5 \times 8$ and $\mu_{y}=2.7+0.3=0.5 \times 6$. This is done by adjusting the strengths of the 10 paired quadrupoles in the "bottom half" of the ring. Since we have retained two-fold symmetry in the ring, the nonlinear magnet can be placed in either $1.8 \mathrm{~m}$ straight section. Figure 4.7 shows the lattice for the one nonlinear magnet experiment configuration.

\subsection{Design of the electron lens Experiment}

The electron lens experiment is the second kind of nonlinear integrable optics test that will take place in the IOTA ring [22], using a pencil beam of $2.5 \mathrm{MeV}$ protons or $150 \mathrm{MeV}$ electrons. This experiment employs a low-energy hollow Gaussian electron beam confined in a solenoidal magnetic field. The core of the circulating pencil beam passes through the center of the electron beam, undisturbed. As in the nonlinear magnet experiments, the electron lens must have a round transverse profile; this means that in the interaction section, the pencil beam must have equal horizontal and vertical beta functions (i.e.. $\beta_{x}=\beta_{y} \equiv \beta$ ), and the dispersion and its derivative must be zero $\left(D_{x}=D_{X}^{\prime}=0\right)$. The nonlinear electromagnetic field generated by the electron distribution retains integrability. A large tune spread is achievable with an electron lens and is given by [22]

$$
\Delta \nu=\frac{\beta j_{0} L\left(1 \pm \beta_{e} \beta_{z}\right)}{2(B \rho) \beta_{e} \beta_{z} c^{2}}\left(\frac{1}{4 \pi \epsilon_{0}}\right),
$$




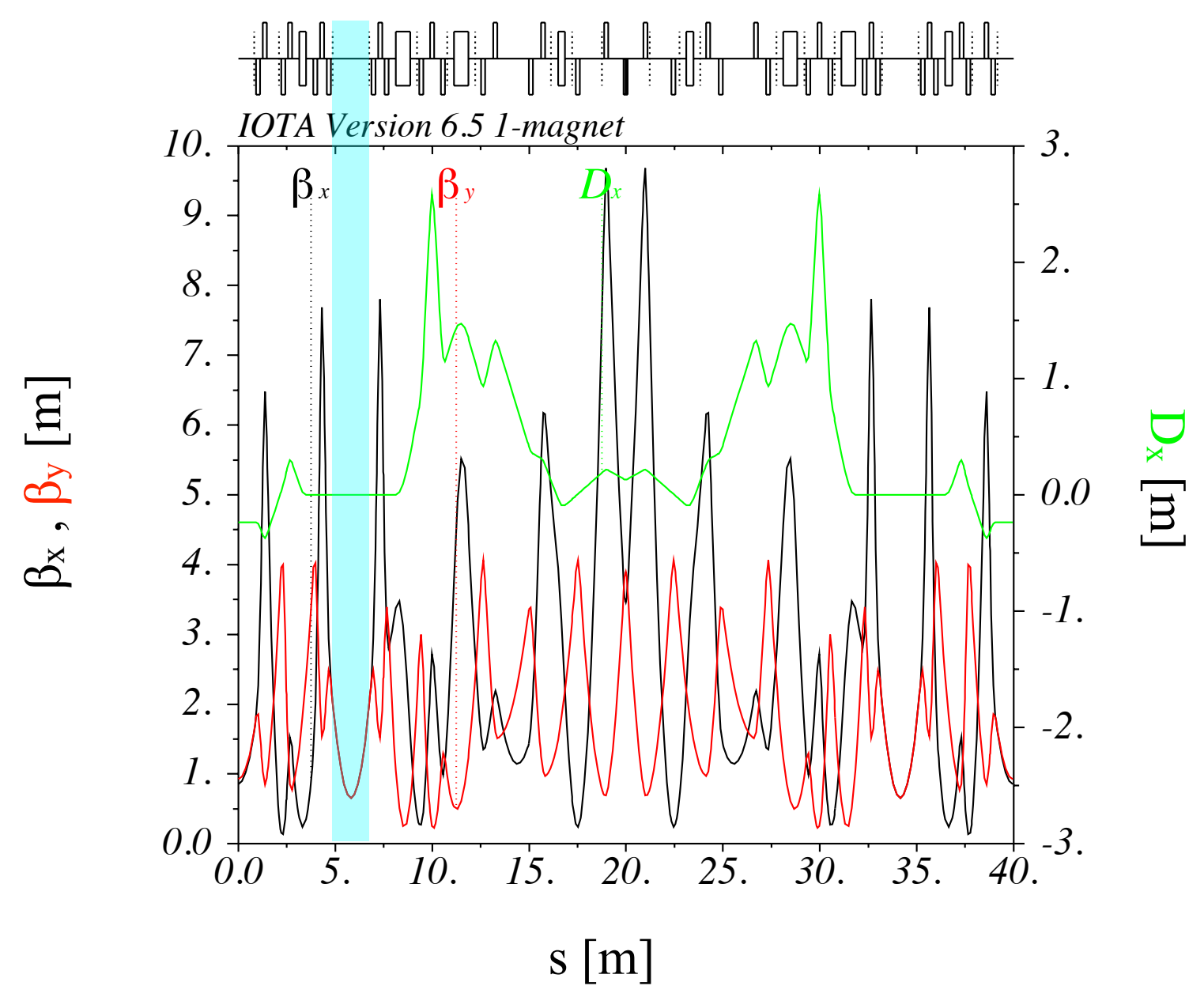

Figure 4.7. IOTA lattice with one nonlinear magnet insert. $\beta_{x}$ and $\beta_{y}$ are in black and red respectively; horizontal dispersion is in green. The nonlinear magnet is marked in cyan.

where $L$ is the interaction length of the pencil beam with the electron lens (approximately equal to the length of the solenoid), $\beta_{e}$ and $\beta_{z}$ are the ratios of the velocities of the electrons comprising the lens and pencil beam with respect to the speed of light, $(B \rho)$ is the magnetic rigidity of the circulating pencil beam, and $j_{0}$ is the current density of the electron-lens beam. The tune spread and dynamic aperture of the ring in the electron lens configuration will be calculated by measuring the position and intensity of the circulating pencil beam. The BPM sensitivity requires a beam envelope larger than $0.1 \mathrm{~mm}$. Table 4.1 lists the parameters for the electron lens configuration 
Table 4.1. Electron Lens Beam Parameters for 2.5 MeV Protons

\begin{tabular}{ccc}
\hline \hline Parameter & Value & Units \\
\hline Energy $E_{0}$ & 2.5 & $\mathrm{MeV}$ \\
Beta Functions Inside electron lens $\beta_{x}=\beta_{y}$ & 3 & $\mathrm{~m}$ \\
Betatron Tune $Q_{x}, Q_{y}$ & $3.5,3.0$ & $2 \pi$ \\
Horizontal Equilibrium Emittance $\epsilon_{x}$ & 0.1 & $\mu \mathrm{m}$ \\
Solenoid Axial Field $B_{z}=\frac{2 B \rho}{\beta}$ & 0.33 & $\mathrm{~T}$ \\
\hline
\end{tabular}

of the IOTA lattice. Figure 4.8 shows the $x$ and $y$ beta functions and the horizontal dispersion for the electron lens configuration.

On close inspection of Figure 4.8, the lattice is seen to be symmetric in the "upper half" of the ring - in the figure it is the section to the left of the electron lens insert and to the right of the same location on the opposite side. Symmetry is not preserved in the "bottom half" of the ring. Since only one side of the ring contains a solenoid, and in order for the beta functions to match before entering the "top half" of the ring, four of the coupled power circuits need to be broken up. If the ring were not so magnetically sparse in this region, only an additional two circuits would be needed to meet all the constraints.

\subsection{Quadrupole Currents}

The final layout of the ring comprises 8 sector dipoles and 39 quadrupoles. 38 of the quadrupoles are arranged in 19 "families," with 2 quadrupoles and 1 power supply per family. All dipoles are powered with a single power supply. The lattice layout with quadrupole circuits is diagramed in Figure 4.9. Of the 32 Dubna quadrupoles, two will be set aside as spares. These quadrupoles can reach a maximum gradient that exceeds IOTA's requirements by a factor of 2. The remaining 9 quadrupoles (plus two spares) needed to complete the ring will be designed to a reduced specification. The price of each quadrupole scales proportionally with its maximum allowable current. For 


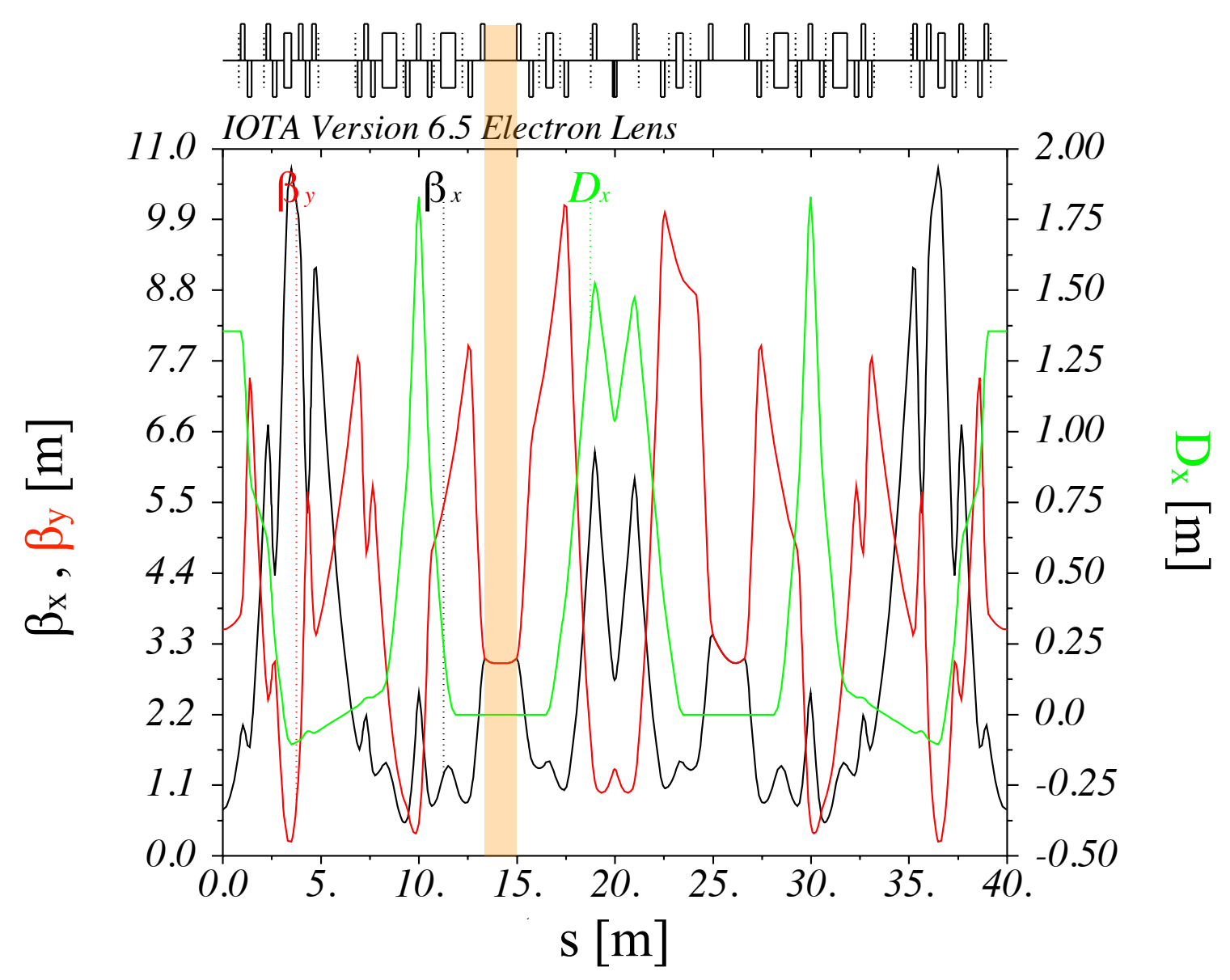

Figure 4.8. IOTA lattice in the electron lens configuration. $\beta_{x}$ and $\beta_{y}$ are in black and red respectively; horizontal dispersion is in green. The electron lens is marked in orange.

purposes of keeping down costs, we have identified the quadrupoles which require the weakest gradients - and consequently the lowest currents - so that these quadrupoles may be manufactured more economically. In Table 4.2, the quadrupole circuits have been sorted according to their largest operating gradient out of all the experiment configurations. From the gradient of each quadrupole $G_{q}$, we can calculate the current, $I$, needed for each quadrupole family:

$$
I=\frac{G_{q}}{N} \frac{R^{2}}{2 \mu_{0}},
$$


Table 4.2. IOTA quadrupole gradients, currents and voltages. The circuit names are congruent with those used in Figure 4.9.

\begin{tabular}{cccccc}
\hline \hline Circuit & Min & Max & Min & Max & Max \\
Name & Gradient $[\mathrm{kG} / \mathrm{cm}]$ & Gradient $[\mathrm{kG} / \mathrm{cm}]$ & Current $[\mathrm{A}]$ & Current $[\mathrm{A}]$ & Voltage $[\mathrm{V}]$ \\
\hline Q18 & 0.019 & 0.249 & 3.0 & 39.6 & 1.6 \\
Q19 & 0.208 & 0.273 & 33.1 & 43.4 & 1.7 \\
Q17 & 0.083 & 0.283 & 13.2 & 45.0 & 1.8 \\
Q16 & 0.019 & 0.284 & 3.0 & 45.2 & 1.8 \\
Q14 & 0.173 & 0.333 & 27.5 & 53.0 & 2.1 \\
Q20 & 0.282 & 0.34 & 44.9 & 54.1 & 2.2 \\
Q15 & 0.061 & 0.38 & 9.7 & 60.5 & 2.4 \\
Q07 & 0.197 & 0.41 & 31.3 & 65.2 & 2.6 \\
Q08 & 0.023 & 0.413 & 3.7 & 65.7 & 2.6 \\
Q01 & 0.18 & 0.452 & 28.6 & 71.9 & 2.9 \\
Q05 & 0.249 & 0.544 & 39.6 & 86.5 & 3.5 \\
Q10 & 0.229 & 0.597 & 36.4 & 95.0 & 3.8 \\
Q12 & 0.42 & 0.605 & 66.8 & 96.3 & 3.9 \\
Q02 & 0.25 & 0.645 & 39.8 & 102.6 & 4.1 \\
Q11 & 0.315 & 0.659 & 50.1 & 104.8 & 4.2 \\
Q03 & 0.09 & 0.661 & 14.3 & 105.2 & 4.2 \\
Q13 & 0.2123 & 0.684 & 33.8 & 108.8 & 4.4 \\
Q09 & 0.175 & 0.716 & 27.8 & 113.9 & 4.6 \\
Q06 & 0.42 & 0.8 & 66.8 & 127.3 & 5.1 \\
Q04 & 0.023 & 1.03 & 3.71 & 63.9 & 6.6 \\
\hline & & & & & \\
\hline
\end{tabular}

where $N$ is the number of turns around each pole, $R$ is the radius from the tip of the pole to the center of the beam pipe, and $\mu_{0}$ is the permeability of the pole. The currents for each quadrupole family are included in Table 4.2. We can see that quadrupole families Q18-Q01 can be made smaller to accommodate a lower required current $(\approx 40 \%$ of what is required in the strongest quadrupole, Q04). A lattice solution that satisfies the criteria for the electron lens experiment found in the previous section requires the addition of four power supplies where the ring is magnetically sparse as seen at the bottom of Figure 4.9 . 


\subsection{RF Considerations}

A particle in a storage ring must stay within a small energy range lest it be lost from the beam. The phase space portrait of a particle inside the RF bucket is bounded by a separatrix. Particles with too high a momentum deviation, or without the proper phase when entering the RF, are eventually lost. A graphical representation of this can be seen in Figure 4.10. The maximum deviation a particle can have without being lost is called the energy acceptance of a storage ring; it is limited by: 1 . the RF acceptance, set by the RF voltage, which at large energy deviations fails to restore the particle energy; and 2. the dynamic acceptance, which shrinks with energy deviation because of chromatic and nonlinear effects. To find the RF acceptance, we recall the solutions found in Eq. 2.44 for the longitudinal motion of a particle in a storage ring. These solutions trace out the separatrices (indicated in blue in Figure 4.10) which intersect at unstable fixed points along a line in the phase space portrait where the Hamiltonian is constant. The RF acceptance is then found by: 1. determining the unstable fixed points using $\frac{d z}{d t}=\frac{d \delta}{d t}=0$ along the separatrix, 2. substituting these points back into the Hamiltonian to find the value of the Hamiltonian along the separatrix, and 3. determining the energy deviation for which $\frac{d \delta}{d t}=0$. Following these steps, we find that the RF acceptance is given by

$$
|\delta|_{\max , R F}=\frac{2 \nu_{s}}{h \alpha_{p}} \sqrt{1-\left(\frac{\pi}{2}-\phi_{s}\right) \tan \phi_{s}},
$$

where $h$ is the cavity harmonic number, $\nu_{s}$ is the synchrotron tune (given in Eq. 2.42), $\alpha_{p}$ is the momentum compaction in the ring, and $\phi_{s}$ is the synchrotron phase, given by

$$
\phi_{s}=\pi-\sin ^{-1}\left(\frac{U_{0}}{q V_{R F}}\right),
$$

where $U_{0}$ is the energy loss per turn and $q V_{R F}$ is the energy restored by the RF cavities. The values of $\nu_{s}$ and $\alpha_{p}$ specific to IOTA are readily available as outputs from MadX and OptiM. They are internally calculated by integrating the Twiss param- 
eters around the ring. To find an RF voltage and harmonic number that yield an acceptable RF acceptance and synchrotron tune, we plot the latter two parameters against the RF voltage for several harmonic numbers in Figure 4.11. The RF acceptance and synchrotron tune for a harmonic number of eight are shown in red and blue respectively.

\subsection{IOTA Parameters}

Table 4.3 summarizes the beam parameters for the nonlinear experiments in the IOTA ring which we have outlined in the previous sections. The momentum compaction, horizontal emittance and bunch length were calculated in OptiM and MadX by integrating the lattice functions around the ring as described in Chapter 2 . Table 4.3 lists the main parameters of the machine for the integrable optics and OSC experiments. 
Table 4.3. IOTA Beam Parameters for $150 \mathrm{MeV}$ Electrons

\begin{tabular}{ccc}
\hline \hline Parameter & Value & Units \\
\hline Energy $E_{0}$ & 150 & $\mathrm{MeV}$ \\
Circumference C & $\approx 40$ & $\mathrm{~m}$ \\
Bending Field & 0.7 & $\mathrm{~T}$ \\
Beam Pipe Aperture (Diameter) & 50 & $\mathrm{~mm}$ \\
Max Betatron Amplitude A & $0.01(\mathrm{IO}) / 0.001$ (OSC) & $\mathrm{m}$ \\
Betatron Function $\beta$ & $3-5$ & $\mathrm{~m}$ \\
Betatron Tune (for one nonlinear insert) & $Q_{x}=Q_{y}=5.3$ & $2 \pi$ \\
Betatron Tune (for two nonlinear inserts) & $Q_{x}=Q_{y}=5.1$ & $2 \pi$ \\
Momentum Compaction $\alpha_{p}$ & $0.06-0.08$ & - \\
Horizontal Emittance $\epsilon$ & $0.03-0.1$ & $\mu \mathrm{m}$ \\
RF Voltage & 1 & $\mathrm{kV}$ \\
RF Harmonic $\left(T_{\text {revolution }} / T_{R F}\right)$ & 4 & $\mathrm{MHz}$ \\
RF Frequency & 30 & - \\
Synchrotron Radiation Tune $Q_{s}$ & 0.005 & $\mathrm{sec}$ \\
Damping Time $\tau$ & 1.7 & - \\
Equilibrium Energy Spread $\sigma_{E} / E$ & $1.3 \times 10^{-4}$ & 0.2 \\
\hline Bunch Length & & $\mathrm{m}$
\end{tabular}




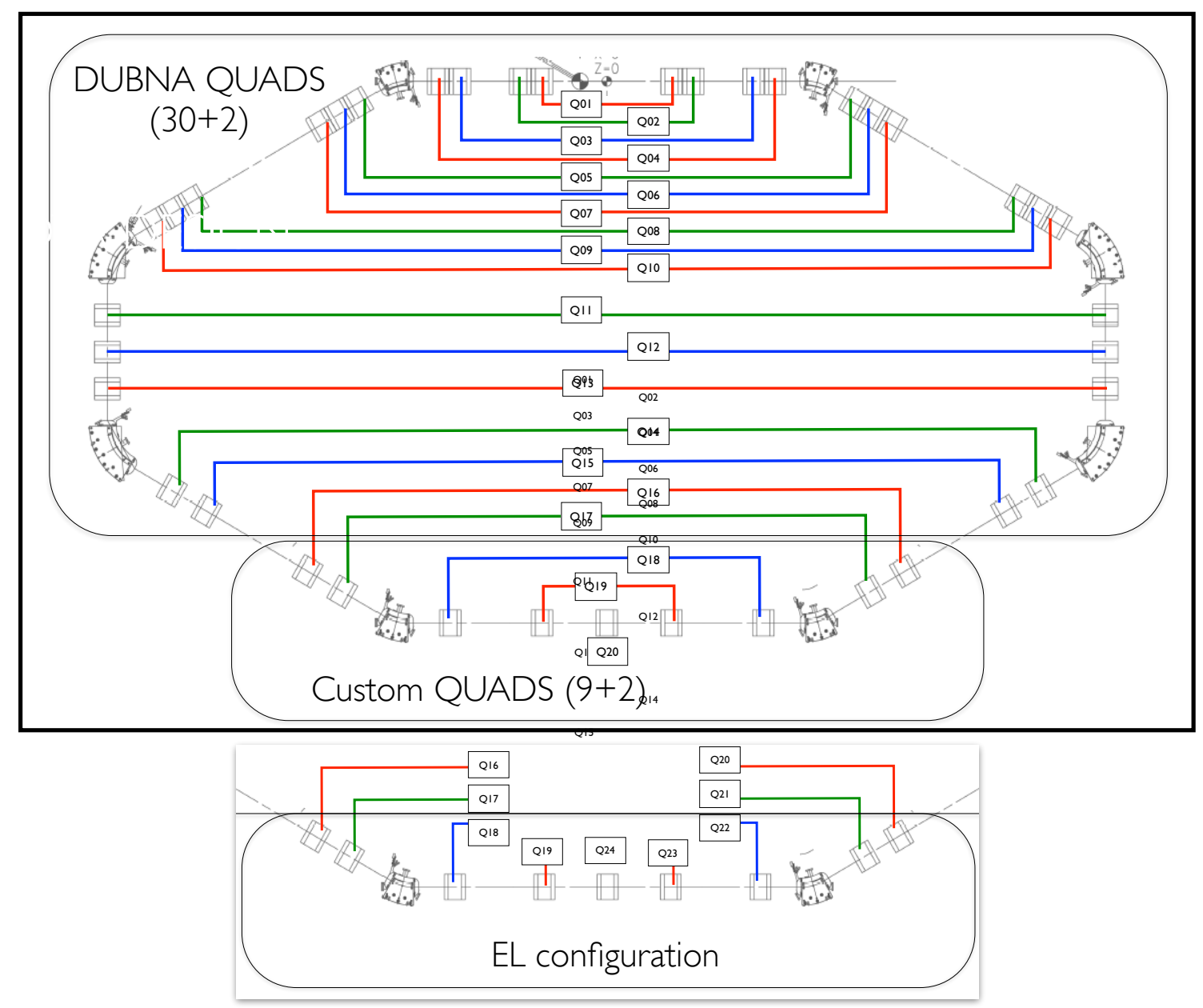

Figure 4.9. Quadrupole families for the nonlinear integrable experiments configurations. In both configurations the quadrupoles from Dubna will occupy the "top half" of the ring and the custom quadrupoles will occupy the "bottom half." The added power supplies for the electron lens configuration are shown at the bottom. 


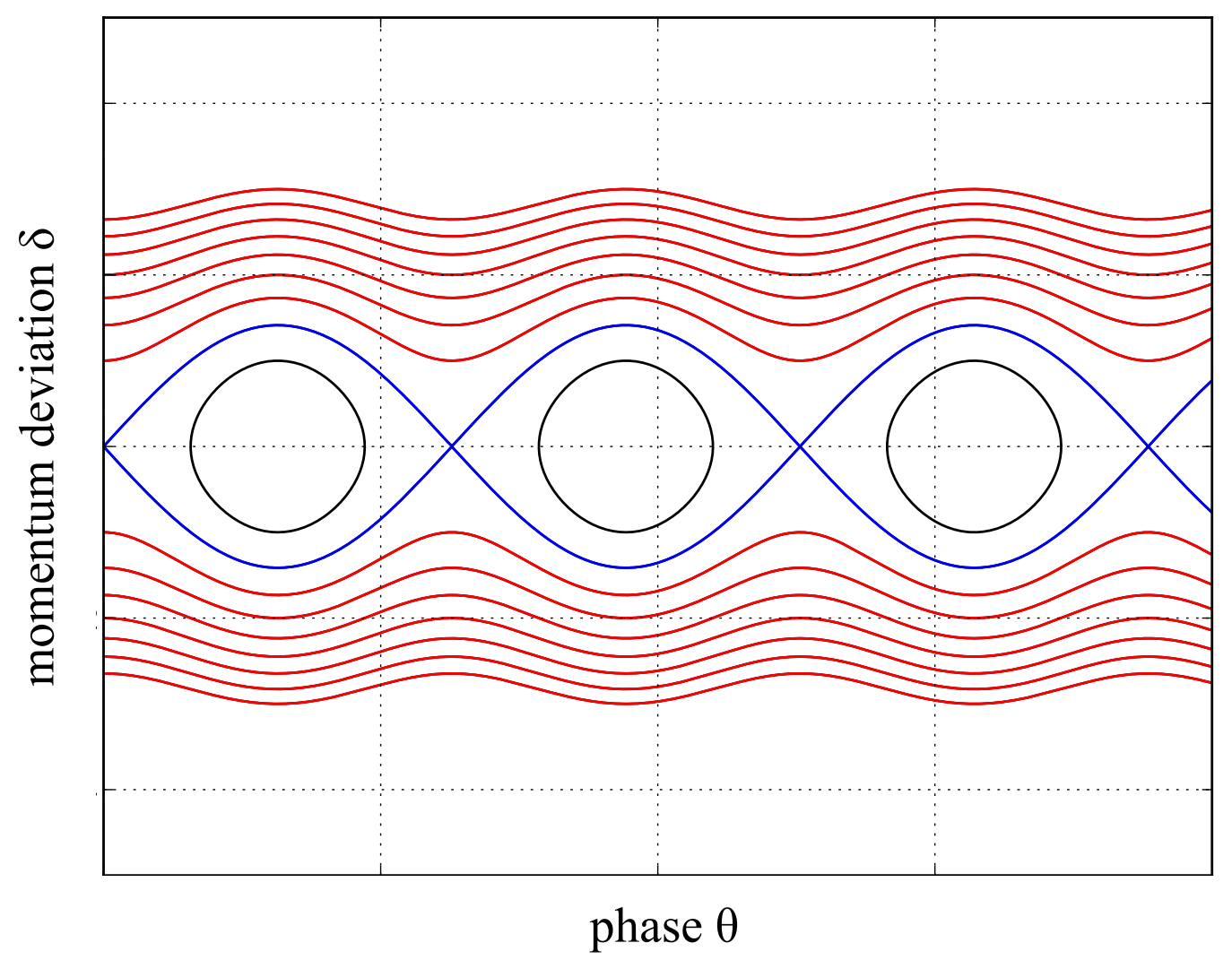

Figure 4.10. The phase space portrait of a particle inside the RF bucket is bounded by a sexparatrix (blue). Particles with too high a momentum deviation, or without the proper phase when entering the RF bucket are eventually lost (red). 


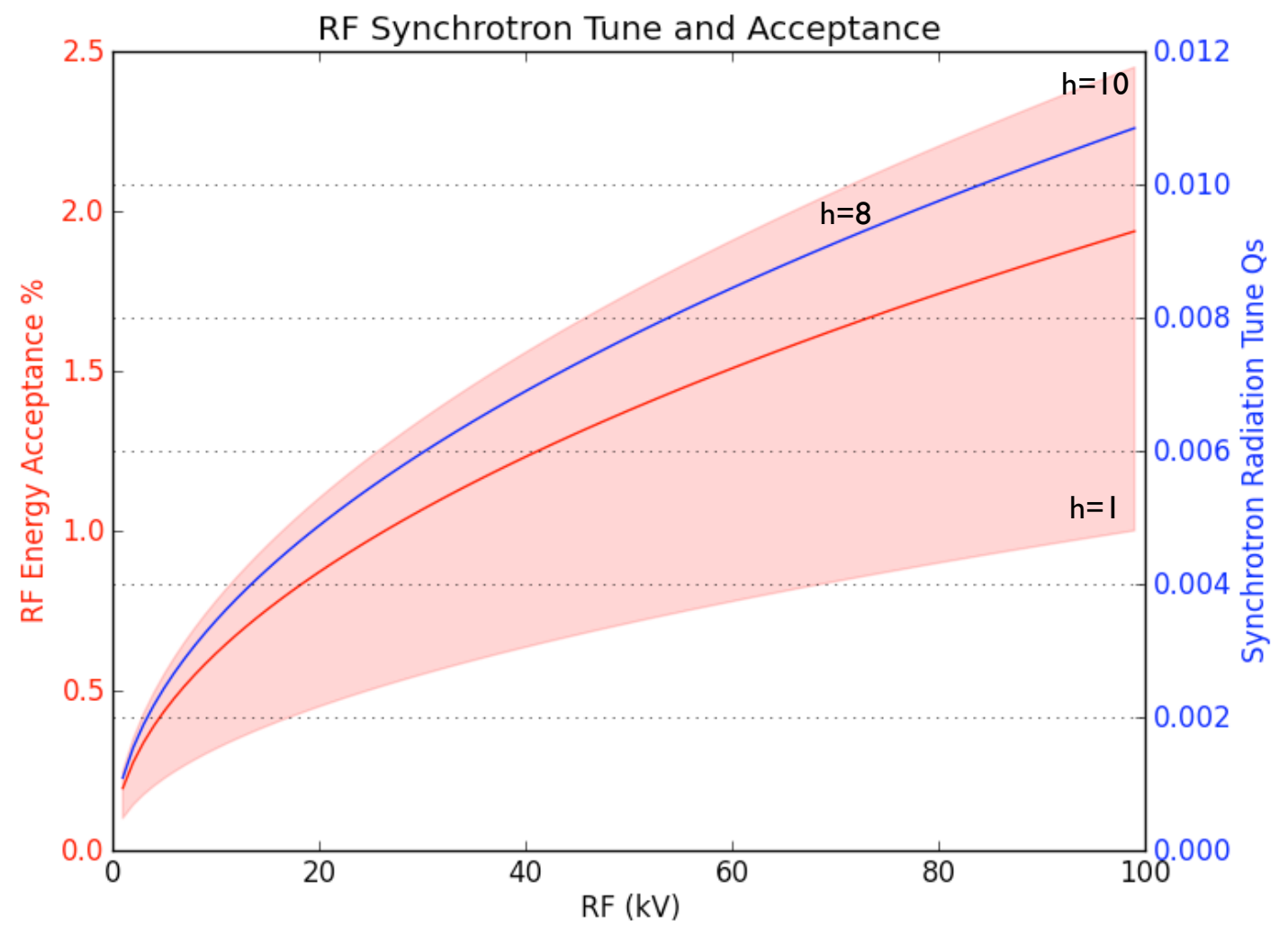

Figure 4.11. RF energy acceptance and synchrotron tune versus RF voltage. The red band outlines the RF energy acceptance for harmonic numbers $h=1 \ldots 10$. The solid red and blue lines show the Rf Acceptance and synchrotron tune for $h=8$ 


\section{CHAPTER 5 \\ OPTICAL STOCHASTIC COOLING IN IOTA}

\subsection{Stochastic Cooling Theory}

In a storage ring, a single particle can easily be cooled via stochastic cooling in the horizontal plane while executing betatron oscillations around the central orbit. In this process, the particle's displacement from the central orbit is measured at the pickup and then amplified and relayed to deflector plates where a kick is applied to the particle's momentum. The effect of the kick reduces the amplitude of the betatron oscillation. As seen in Figure 5.1, a pickup is placed where the horizontal position is at a maximum displacement from the central orbit, and the most effective kick is placed where the trajectories are maximally divergent and coincident with the central orbit. This scenario requires that the phase advance between the pickup and kicker be $0.25 n$ (in units of $2 \pi$ ), where $n$ is odd.

Suppose a collection of $N$ particles is making betatron oscillations around a storage ring, and at some time when the average displacement is not along the reference orbit $(\bar{x}(t) \neq 0)$, a kick is applied to the bunch to correct for a particle $i$. It corrects the horizontal position of particle $i$ by [23]

$$
\Delta x(t)=-\frac{g}{N} x_{i}(t)-\frac{g}{N} \sum_{k \neq i}^{N} x_{k}(t)
$$

where $g$ is the gain factor. The first term is the kick generated by the particle itself at the pickup, and always damps (cools) its motion; the second term is the kick generated by the rest of the bunch, denoted by subscript $k$, and does not necessarily drive the damping (possibly heats). 


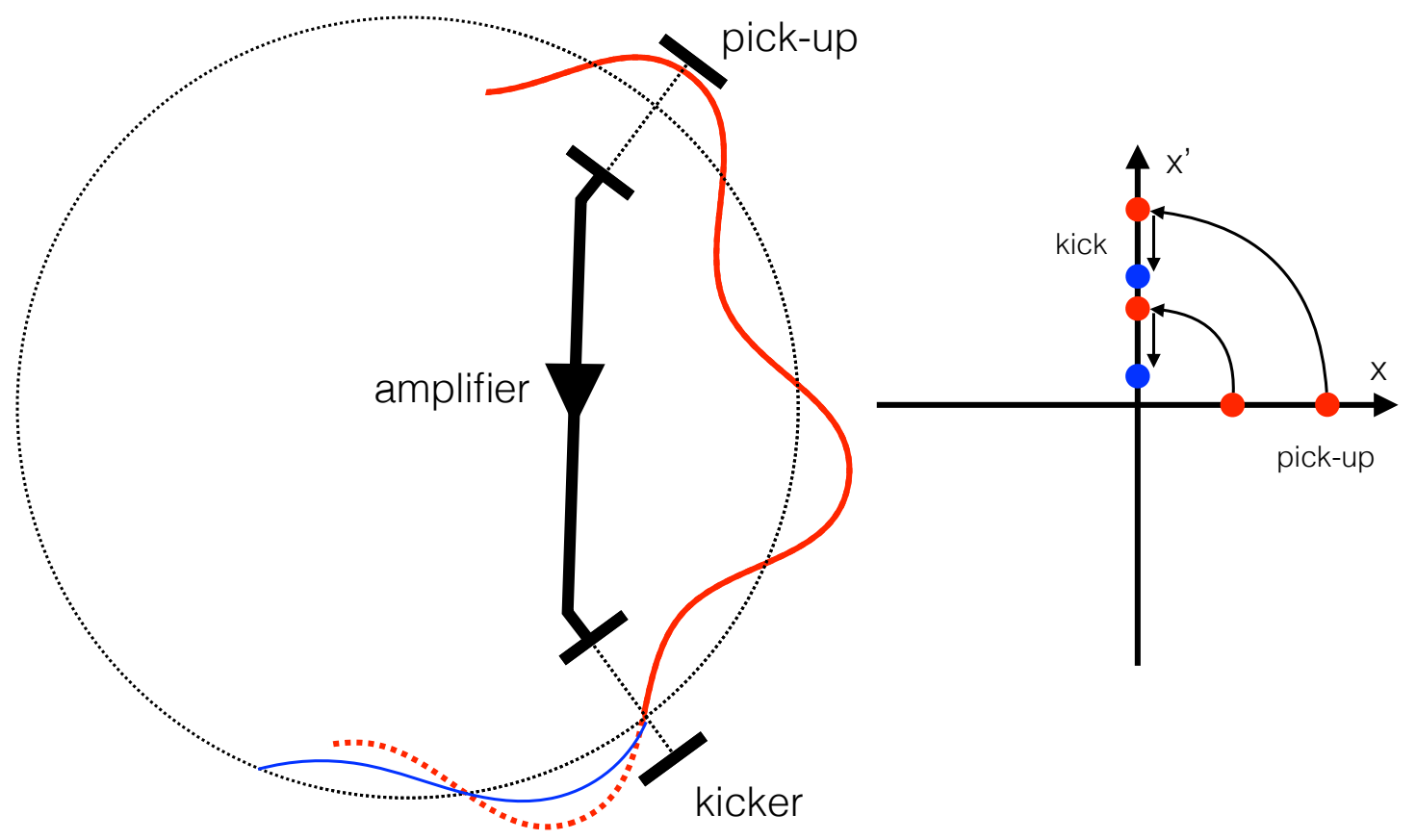

Figure 5.1. Horizontal damping of betatron oscillations via a pick-up and kicker with a phase advance of 1.75 . The right diagram shows the phase-space coordinates of a beam damped with a phase advance of 0.25 between the pickup and kicker.

The amplitude of each oscillator is now dependent on time, and the RMS amplitude is simply [23]

$$
\sigma^{2}(t)=\left\langle\overline{x^{2}}(t)\right\rangle=\frac{1}{2 N} \sum_{i=1}^{N} A_{i}^{2}(t)=\frac{1}{2 N} \sum_{i=1}^{N}\left[x_{i}(t)+\Delta x(t)\right]^{2}-x_{i}^{2}(t),
$$

where $A_{i}(t)$ is the amplitude of the $i$ th particle and $\Delta x(t)$ is defined in Equation 5.1. To find the average change in amplitude at time $t+\tau$, we integrate the previous equation over time and get

$$
\left\langle\Delta A_{i}^{2}(t, \tau)\right\rangle=-\frac{2 g}{N} \frac{A_{i}^{2}(t)}{2}+\frac{g^{2}}{N^{2}} \sum_{j=1}^{N} \frac{A_{j}^{2}(t)}{2} .
$$

Finally, the change in the RMS amplitude can be expressed by summing over the time averaged change in amplitude to arrive at an equation for cooling (or heating) that relies only on the gain of the system and the initial RMS amplitude of the beam 


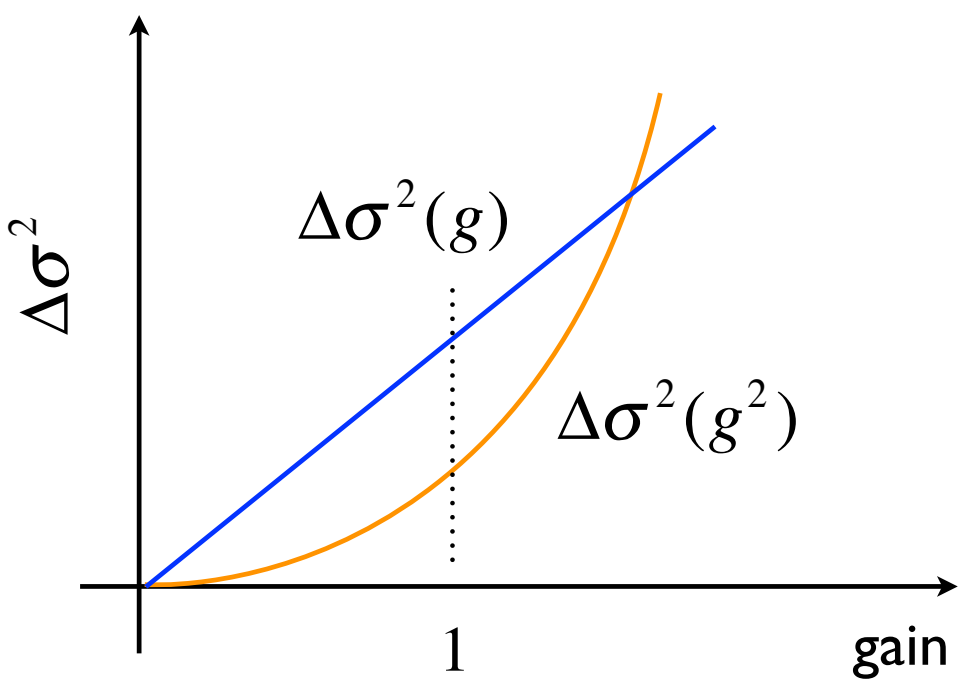

Figure 5.2. Heating (orange) and cooling (blue) contributions to stochastic cooling. The optimal gain is marked by the dotted line.

$[23]:$

$$
\begin{aligned}
\Delta \sigma^{2}(t) & =\sum_{i=1}^{N}\left\langle\Delta A_{i}^{2}(t, \tau)\right\rangle \\
& =-\frac{2 g}{N} \sum_{i=1}^{N} \frac{A_{i}^{2}(t)}{2}+\frac{g^{2}}{N^{2}} \sum_{i=1}^{N} \sum_{j=1}^{N} \frac{A_{j}^{2}(t)}{2} \\
& =\frac{-2 g+g^{2}}{N} \sigma^{2}(t) .
\end{aligned}
$$

As seen in Figure 5.2, the optimum gain, $g=1$, is the point at which the beam is most effectively cooled. The damping decrement per revolution around the ring for a given number of particles in a sample (within the bandwidth) $N_{s}$ is $\lambda=\frac{1}{N_{s}}$, and the damping rate (or inverse damping time) is [4]

$$
\frac{1}{\tau}=\frac{1}{4 N_{s} T}=\frac{\Delta f}{2 N}
$$

where $\Delta f$ is the bandwidth of the feedback system; a factor 2 accounts for the betatron oscillation not being a maximum at the pick-up, and another factor 2 signifies the damping rate is in terms of amplitude and not its square. This approximation assumes ideal mixing outside of the feedback loop. 


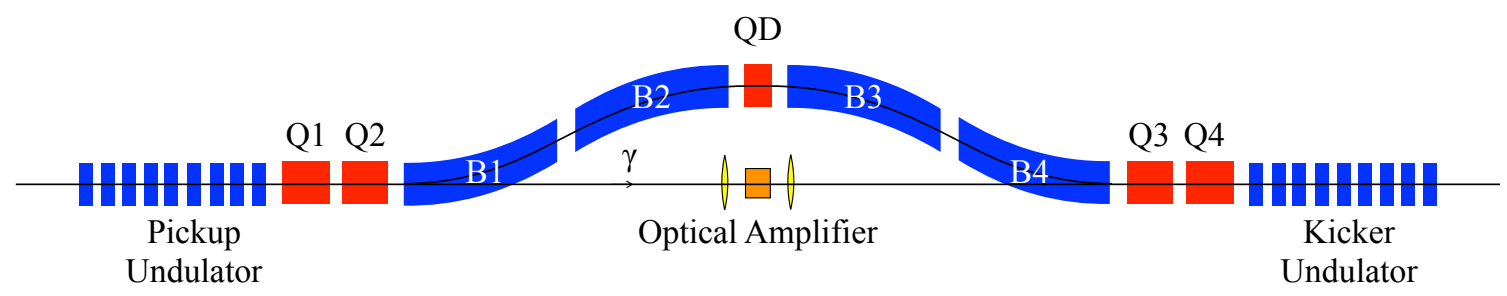

Figure 5.3. The OSC bypass including two undulators, quadrupoles (red) and bending magnets (blue).

\subsection{OSC Bypass Optics}

Conventional stochastic cooling systems with waveguides are limited to a bandwidth $\Delta f \approx 1-10 \mathrm{GHz}$. OSC can provide damping rates 4 orders of magnitude larger than the conventional method by utilizing a superior bandwidth on the order of $10^{14}$ $\mathrm{Hz}$, allowing for finer sampling of the bunch. The OSC system that will be implemented in IOTA is diagramed in Figure 5.3, and consists of two undulators, a bypass made up of four bending magnets, five quadrupoles, and an optical amplifier. OSC works on the same general principle as conventional stochastic cooling, but uses undulators instead of RF pickups and kickers. In this setup, a particle radiates an electromagnetic (EM) wave in the first undulator (as seen in Figure 5.4) that is propagated through an optical amplifier while the particle is directed though a bypass. The time of the amplification process is matched by the transit time of the particle through the bypass. In the second undulator, the particle interacts with its own amplified radiation, which results in an energy change that is proportional to the phase of the EM wave as it interacts with the particle. The radiation generated in the first undulator by particle $i$ is propagates in the longitudinal plane and is given by [24]

$$
E_{i}=E_{0} \sin \left(k z-\omega t+\phi_{i}\right)
$$

where $E_{0}$ and $\phi_{i}$ are the amplitude and phase of the electric field. The wave number and frequency are $k=\frac{2 \pi}{\lambda}$ and $\omega=k c$. The wavelength can be found in terms of both 


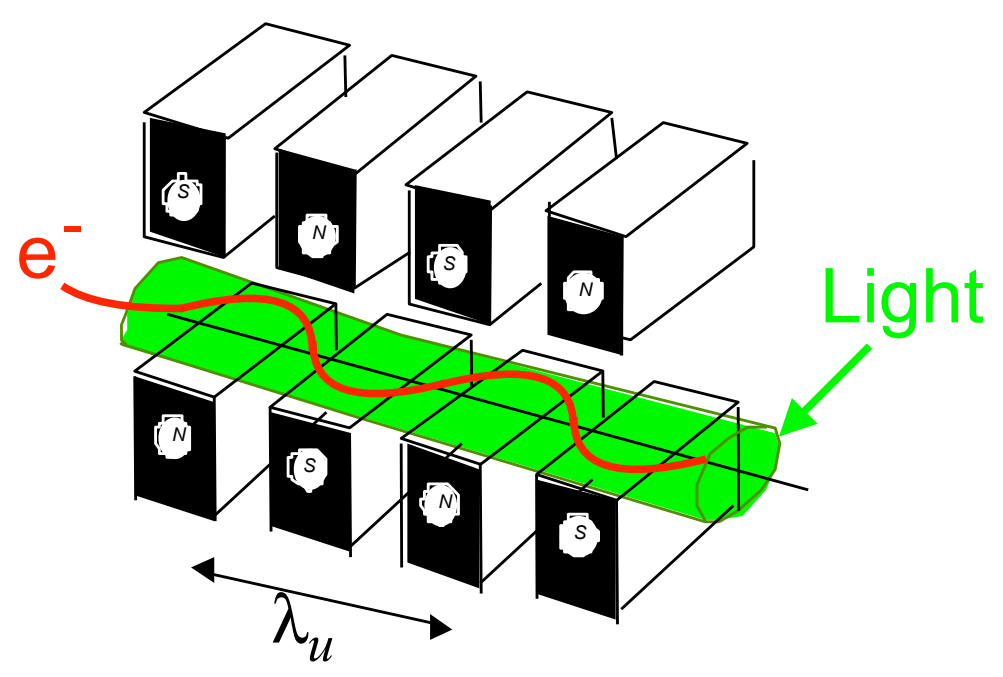

Figure 5.4. Particle and radiation in an undulator segment.

the undulator period $\lambda_{u}$ and undulator parameter $K_{u}$ as follows [24]:

$$
\lambda=\frac{\lambda_{u}}{2 \gamma^{2}}\left(1+\frac{K_{u}^{2}}{2}\right)
$$

where $\gamma$ here is the Lorentz factor. The undulator parameter $K_{u}$ is defined as

$$
K_{u}=\frac{e B_{u} \lambda_{u}}{2 \pi m c}
$$

where $e$ and $m$ are the electron charge and mass, $B_{u}$ is the magnetic field in the undulator, and $c$ is the speed of light. Figure 5.4 depicts an electron making oscillations with period $\lambda_{u}$ through a segment of the undulator.

The change in longitudinal momentum for the particle due to the kick is similar to that outlined in section 5.1, but now the kick is longitudinal, nonlinear, and depends on the phase difference between the pickup (0) and kicker (1), $\psi_{i k}=\phi_{1}-\phi_{0}$ :

$$
\Delta \delta_{i}=\left(\frac{\Delta p}{p}\right)_{i}=-G \sin \left(k \Delta s_{i}\right)-G \sum_{j \neq i}^{N_{s}} \sin \left(k \Delta s_{i}+\psi_{i j}\right)
$$

where $\Delta s_{i}=\left(s_{1}-s_{0}\right)$ is the longitudinal displacement of the particle from the reference path; the ideal gain $G$ relies on several parameters, some of which will be 
discussed in detail in the following sections. Since all EM waves propagate from undulator to undulator at the same rate, the variation of phases of different particles is due only to the particles' transit times in the bypass. Therefore, in order to correct the particle's momentum deviation, 1. the bypass needs to transport a particle with zero momentum offset so that it will meet the radiation at a phase with zero electric field, and thus not receive a kick, and 2. the bypass must be designed such that the particles' path lengths correspond to their momentum offset in such a way that the interaction with radiation occurs at a phase that is proportional to the value of the momentum deviation.

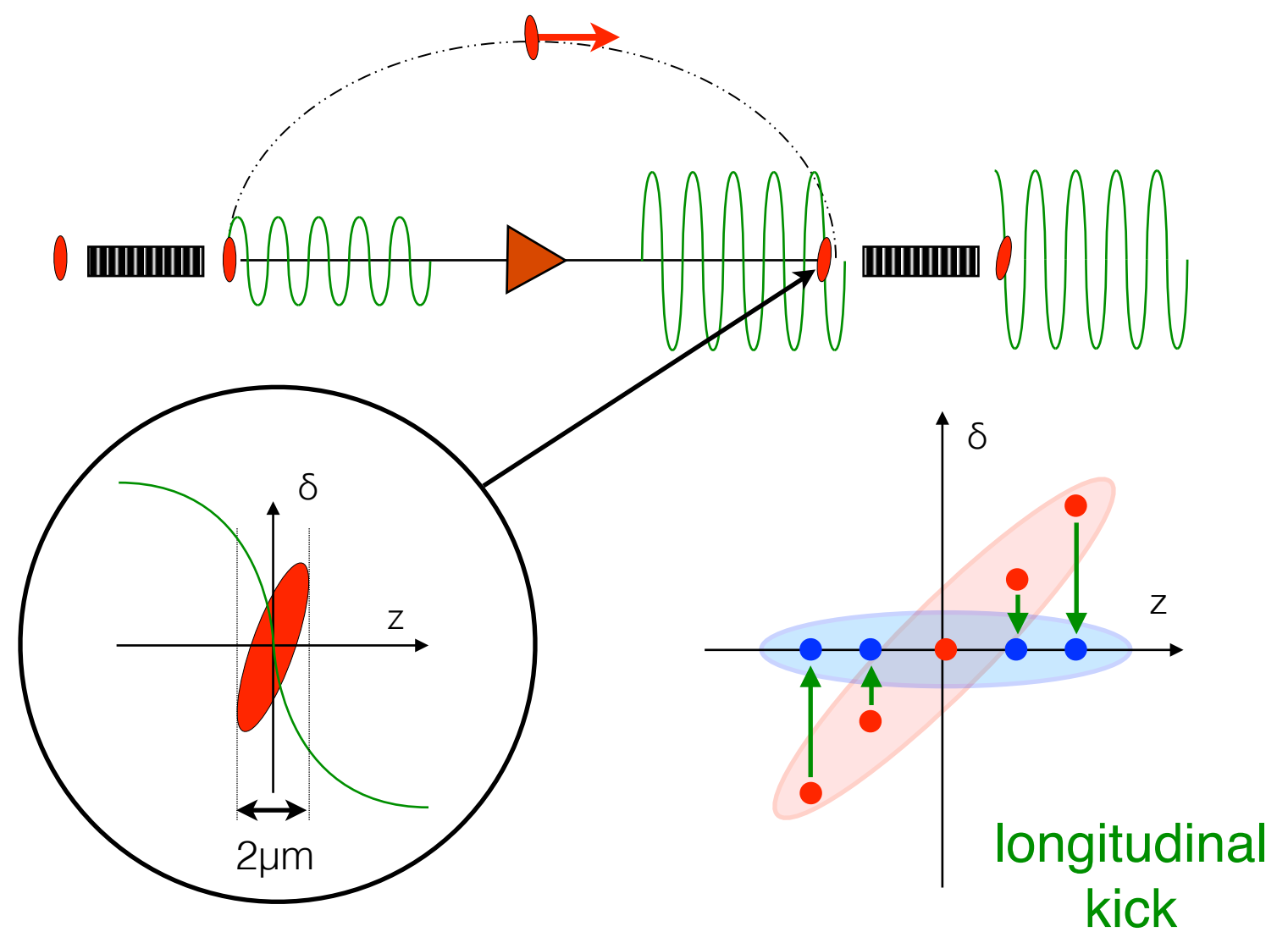

Figure 5.5. An electron bunch radiates in the first undulator, is transported via a bypass and kicked in the second undulator. The kick is proportional to the displacement in $z$. A coherent kick corresponds to a bunch lengthening that is smaller than the wavelength of the radiation. 
Figure 5.5 shows the orientation of the longitudinal phase space of the sample as it traverses the bypass - the faster particles overtake the reference particle and the slower ones lag. The kick in the second wiggler slows the former and speeds up the latter in proportion to their longitudinal offset; as in transverse cooling the kick affects only the momentum coordinate. The sample must not lengthen more than the usable (approximately linear) segment of the radiation wavelength, $\lambda_{R}$. Knowing the undulator wavelength, $\lambda_{u}, \lambda_{R}$ can be approximated as follows:

$$
\lambda_{u}=2 \gamma^{2} \lambda_{R}
$$

where $\gamma$ is the Lorentz factor of the beam. Transverse cooling is achieved through coupling between longitudinal and horizontal motion.

For simplicity, we may treat the vertical plane as uncoupled to the horizontal motion, and keeping only the linear terms in the Taylor expansion of $\sin \left(\Delta s_{i}\right)$ in Equation 5.9, we can use the pickup-to-kicker transfer matrix to transport the particles' horizontal and longitudinal coordinates through the bypass from the pickup (marked with subscript 0) to the kicker (subscript 1):

$$
\left(\begin{array}{c}
x_{1} \\
\left(\theta_{x}\right)_{1} \\
s_{1} \\
(\Delta p / p)_{1}
\end{array}\right)=\left(\begin{array}{cccc}
M_{11} & M_{12} & 0 & M_{16} \\
M_{21} & M_{22} & 0 & M_{26} \\
M_{51} & M_{52} & 1 & M_{56} \\
0 & 0 & 0 & 1
\end{array}\right)\left(\begin{array}{c}
x_{0} \\
\left(\theta_{x}\right)_{0} \\
s_{0} \\
(\Delta p / p)_{0}
\end{array}\right) \text {. }
$$

The matrix transports the vector $\left(x, \theta_{x}, s, \Delta p / p\right)$ for each particle, where $x$ and $\theta_{x}$ are the horizontal position and angle, $s$ is the longitudinal displacement, and $\Delta p / p$ is the momentum offset. The transfer matrix elements $M_{11}, M_{12}, M_{22}$ and $M_{21}$ are identical to the ones found in 2.24, and can be used in conjunction with the dispersion 
functions at the pickup and kicker to describe the rest of the transport matrix:

$$
\begin{aligned}
& M_{16}=D_{1}-M_{11} D_{0}-M_{12} D_{0}^{\prime} \\
& M_{26}=D_{1}^{\prime}-M_{21} D_{0}-M_{22} D_{0}^{\prime} \\
& M_{51}=M_{21} M_{16}-M_{11} M_{26} \\
& M_{52}=M_{22} M_{16}-M_{12} M_{26} .
\end{aligned}
$$

The $M_{5 j}$ elements, where $j=1,2$, describe the longitudinal offset dictated by the particle's horizontal position and angle. $M_{56}$ controls the path lengthening due to momentum deviations $\Delta p / p$ of the electron bunch; $M_{56}$ is positive, meaning that particles with positive $\Delta p$ move ahead of the reference particle. With all matrix elements defined in terms of Twiss parameters and the phase advance, we can first linearize Equation 5.9:

$$
\Delta \delta=G \sin (k \Delta s)=G k \Delta s=G k\left(M_{51} x+M_{52} \theta_{x}+M_{56} \Delta p / p\right)
$$

where $k=2 \pi / \lambda$ is the radiation wave number. The path lengthening, $\Delta s$, in this equation can be rewritten in terms of the Twiss parameters at the beginning and end of the bypass, $\Delta s$ and $M_{56}$ :

$$
\begin{aligned}
& \Delta s=M_{56} \frac{\Delta p}{p} \\
& -D_{0} D_{1}\left(\frac{1+\alpha_{0} \alpha_{1}}{\sqrt{\beta_{0} \beta_{1}}} \sin \mu_{p k}+\frac{\alpha_{1}-\alpha_{0}}{\sqrt{\beta_{0} \beta_{1}}} \cos \mu_{p k}\right)-D_{0} D_{1}^{\prime} \sqrt{\frac{\beta_{1}}{\beta_{0}}}\left(\cos \mu_{p k}+\alpha_{0} \sin \mu_{p k}\right) \\
& \quad+D_{0}^{\prime} D_{1} \sqrt{\frac{\beta_{0}}{\beta_{1}}}\left(\cos \mu_{p k}-\alpha_{1} \sin \mu_{p k}\right)-D_{0}^{\prime} D_{1}^{\prime} \sqrt{\beta_{0} \beta_{1}} \sin \mu_{p k}
\end{aligned}
$$

We will use this relation to find $\Delta s$ at points throughout the bypass. One can use perturbation theory and the symplecticity of the undamped motion to obtain the small amplitude approximation of the horizontal and longitudinal $\left(\lambda_{x}\right.$ and $\left.\lambda_{s}\right)$ cooling 
rates as derived in [25]:

$$
\left(\begin{array}{c}
\lambda_{x} \\
\lambda_{s}
\end{array}\right)=\frac{G k}{2}\left(\begin{array}{c}
M_{56}-2 \pi R \eta_{56} \\
2 \pi R \eta_{56}
\end{array}\right)
$$

where the partial slip factor, $\eta_{56}$, is given by

$$
\eta_{56}=\frac{-1}{2 \pi R}\left(M_{51} D_{0}+M_{52} D_{0}^{\prime}+M_{56}+135 \times 2 / \gamma^{2}\right)
$$

where $R$ is the bending radius of the ring. For a particle without betatron oscillations and with momentum deviation $\frac{\Delta p}{p}$, the longitudinal displacement relative to the ref-

erence particle is equal to $\eta_{56} \frac{\Delta p}{p}$. Here $\gamma$ is the Lorentz factor, and $D_{0}$ and $D_{0}^{\prime}$ are the horizontal dispersion function and its derivative in the pickup, the values of which are set initially by the optics in the bypass, as outlined in Section 5.4. For horizontal damping, $M_{56}$ and $2 \pi R \eta_{56}$ must be different.

\subsection{Path Lengthening in the Bypass}

The pickup-to-kicker path lengthening, $\Delta s$ is due to both betatron and synchrotron oscillations; these contributions can be approximated with the two following equations

$$
\begin{aligned}
\Delta s_{x} & =M_{51} x+M_{52} x^{\prime}, \\
\Delta s_{p_{x}} & =\left(M_{51} D_{x}+M_{52} D_{x}^{\prime}+M_{56}\right) \frac{\Delta p}{p} .
\end{aligned}
$$

Assuming that the beam is a multivariate Gaussian distribution, we can approximate the increase in the standard deviation ${ }^{6}$ in both planes $-\sigma_{x}$ and $\sigma_{\delta}$. A Gaussian beam with the preceding characteristics has the following distribution function:

$$
f\left(x, \theta_{x}, \delta\right)=\frac{1}{(2 \pi)^{3 / 2} \sigma_{x}^{2} \sigma_{\delta}} e^{-\frac{x^{2}+\theta_{x}^{2}}{2 \sigma_{x}^{2}}-\frac{\delta^{2}}{2 \sigma_{\delta}^{2}}},
$$

${ }^{6}$ Adding in quadrature the mean, $\bar{x}$, and the standard deviation, $\sigma_{x}$, yields the square of the RMS: $x_{r m s}^{2}=\bar{x}^{2}+\sigma_{x}^{2}$. 
where $\theta_{x}=\beta x^{\prime}+\alpha x, \theta_{x}$ is the horizontal angle, and $\sigma_{x}$ and $\sigma_{\delta}$ are the horizontal and longitudinal beam sizes. To determine the RMS lengthening of a zero-length sample from pickup to kicker we evaluate the path-length Gaussian integral:

$$
\sigma_{\Delta s}^{2}=\iiint \Delta s^{2} f\left(x, \theta_{x}, \delta\right) d x d \theta_{x} d \delta
$$

Upon integration, we see that the sample lengthening, $\sigma_{\Delta s}$, caused by the momentum offset $\left(\sigma_{\Delta s p}\right)$ and the transverse emittance $\left(\sigma_{\Delta s \epsilon}\right)$ are given by

$$
\begin{aligned}
& \sigma_{\Delta s \epsilon}^{2}=\epsilon_{x}\left(\beta_{0} M_{51}^{2}-2 \alpha_{0} M_{51} M_{52}+\gamma_{0} M_{52}^{2}\right) \\
& \sigma_{\Delta s p}^{2}=\sigma_{p}^{2}\left(M_{51} D_{0}+M_{52} D_{0}^{\prime}+M_{56}\right)^{2} .
\end{aligned}
$$

Here we have used the following phase ellipse relations to express the lengthening equations in terms of the Twiss parameters and beam size:

$$
\left(\begin{array}{c}
\beta_{x} \\
\alpha_{x} \\
\gamma_{x}
\end{array}\right)=\left(\begin{array}{c}
\left\langle x_{0}^{2}\right\rangle / \epsilon_{x, r m s} \\
-\left\langle x_{0} x_{0}^{\prime}\right\rangle / \epsilon_{x, r m s} \\
\left\langle x_{0}^{\prime 2}\right\rangle / \epsilon_{x, r m s}
\end{array}\right) .
$$

The addition of the two equations in 5.21 gives the total RMS sample lengthening in the bypass: $\sigma_{\Delta s}^{2}=\sigma_{\Delta s \epsilon}^{2}+\sigma_{\Delta s p}^{2}$. The sample lengthening in the bypass is plotted in Figure 5.6. One can see as illustrated in this figure that the linear design of the bypass has large cancellations in the sample lengthening through the chicane, and upon exiting the bypass, the sample will have grown and lost two orders of magnitude in length. For this design, the sample lengthening at the exit of the bypass is $\approx 0.3$ $\mu \mathrm{m}$

\subsection{Optics Control in the Bypass}

For effective transverse cooling, $M_{56}$ and $2 \pi R \eta_{56}$ must be different (see Eq. 5.15) [26]. This is achieved by placing a defocusing quadrupole in the center of the bypass. The length of the quadrupole is kept short to help reduce the sample lengthening through the bypass. As usual, its strength is inversely proportional to 


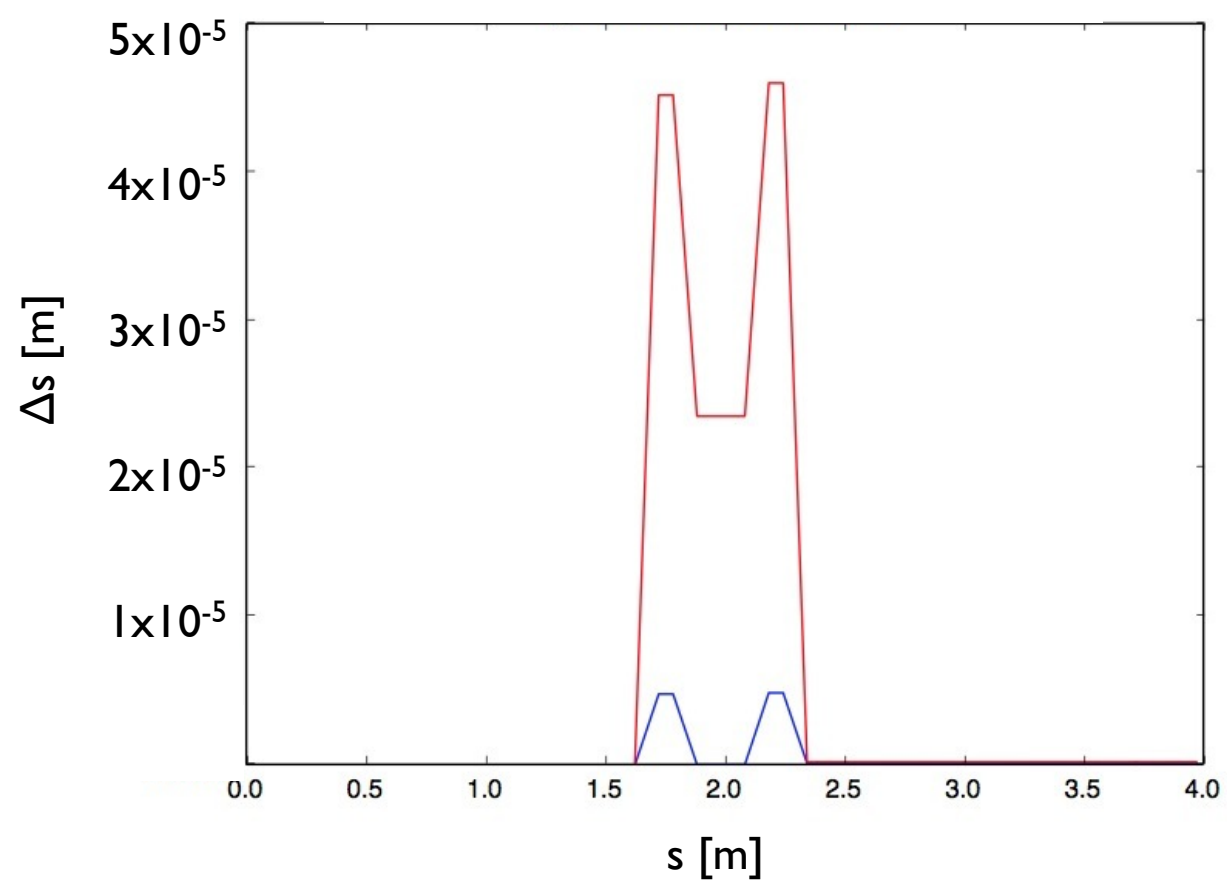

Figure 5.6. Theoretical sample lengthening due to horizontal (red) and momentum offset (blue) through the bypass

its focal length: $\Phi=1 / F$. To minimize the equilibrium beam emittance, we require that the horizontal dispersion is flat in the center of the bypass, i.e., $D_{x}^{\prime}=0$. The Twiss parameters $\beta_{x}, \beta_{y}$ and $D_{x}$ for the bypass are depicted in Figure 5.7. Keeping only leading terms in the thin lens approximation, we have [26]:

$$
\begin{aligned}
M_{56} & \approx 2 \Delta s, \\
\eta_{56} & \approx 2 \Delta s-\Phi D^{*} h, \\
\lambda_{x} / \lambda_{s} & \approx \Phi D^{*} h /\left(2 \Delta s-\Phi D^{*} h\right),
\end{aligned}
$$

where $h$ is the horizontal displacement provided by the bypass. The quantity $\Phi D^{*} h$ determines the cooling dynamics and will be used to set the beta function and dispersion values in the bypass.

To determine the cooling area boundaries, i.e., the maximum allowable horizontal and longitudinal deviations above which cooling cannot be achieved, we use 


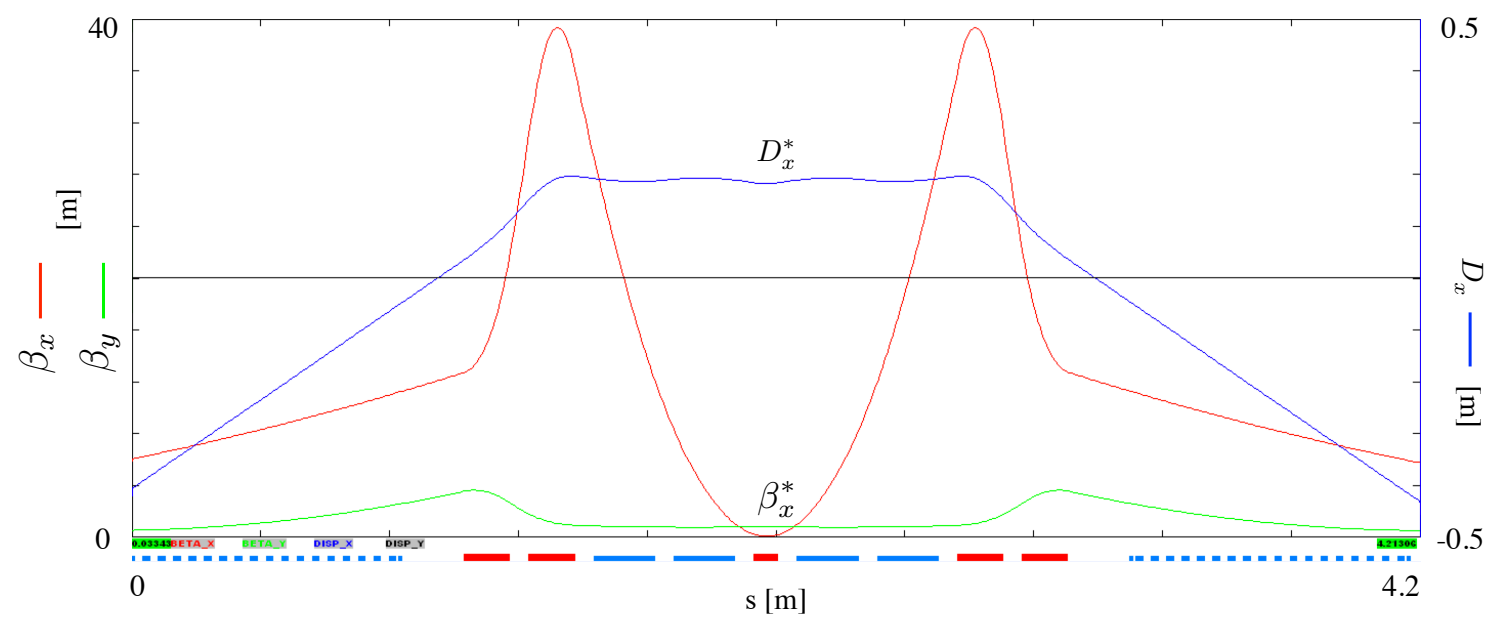

Figure 5.7. The beta functions $\beta_{x}, \beta_{y}$ (red and green respectively) and the horizontal dispersion (blue) are shown here for the OSC bypass.

Eqs. 5.21:

$$
\begin{aligned}
\sigma_{x}^{\max } & =\frac{A_{0}^{2}}{\left(\beta_{0} M_{51}^{2}-2 \alpha_{0} M_{51} M_{52}+\gamma_{0} M_{52}^{2}\right)} \\
\left(\sigma_{s}^{\max }\right)^{2} & =\frac{A_{0}^{2}}{\left(M_{51} D_{0}+M_{52} D_{0}^{\prime}+M_{56}\right)},
\end{aligned}
$$

where $A_{0}$ is the amplitude at which the damping rates change signs if exceeded [26]. The cooling ranges $n_{\sigma s}$ and $n_{\sigma x}$ are then defined as the areas of the beam that can be cooled. They can be expressed in terms of the bypass parameters:

$$
\begin{aligned}
& n_{\sigma s}=\frac{\delta_{s}^{\max }}{\sigma_{p}}=\frac{A_{0}}{k \sigma_{p}\left(2 \Delta s-\Phi D^{*} h\right)} \\
& n_{\sigma x}=\sqrt{\frac{\epsilon_{x}^{\max }}{\epsilon}}=\frac{A_{0}}{2 k h \Phi \sqrt{\epsilon_{x} \beta^{*}}},
\end{aligned}
$$

where $\beta^{*} \approx \frac{L^{2}}{\beta}$ is the beta function at the center of the $2 L$-long bypass. Using the second equation in 5.27, we finally get:

$$
\Phi D^{*} h \approx \frac{A_{0}}{2 k n_{\sigma x}} \sqrt{\frac{\mathcal{H}^{*}}{\epsilon}},
$$

where the dispersion invariant at the center of the bypass is simply $\mathcal{H}^{*}=\frac{D^{*}}{\beta^{*}}$. With the above relations, we can determine the optimum values for Twiss parameters in the bypass. 
For the OSC configuration as well as for the integrable optics experiments, the ring is designed to operate at the coupling resonance, i.e., where the horizontal and vertical tunes are equal or differ by an exact integer. By doing so, the synchrotron radiation cooling rates can be redistributed between the horizontal and vertical planes evenly resulting in equal equilibrium transverse emittances that are twice as small as the undistributed horizontal emittance [27]. In IOTA, OSC will be done in two phases, the first of which will not include an optical amplifier. During this phase, a damping rate higher than the cooling rate due to synchrotron radiation will be needed so that the effects of optical stochastic cooling can be measured. As shown in the following sections, the damping time for OSC is several orders of magnitude smaller than that of synchrotron radiation.

\subsection{Sextupole Placement in the Bypass}

In order to correct for the sample lengthening, we can place sextupoles in the bypass between the dipoles where the dispersion is high. The nonlinear kicks from two sextupoles $S 1$ and $S 2$ of the same strength are separated by a phase advance $\Delta \mu$ of an odd multiple of $\pi$. The nonlinear kicks in this sextupole configuration will cancel out if there are no other nonlinear elements between them [28]. Figure 5.8 shows the effect of these kicks on the betatron oscillation. For a particle with a nonzero momentum offset, i.e., $\delta \neq 0$, the tune is shifted: $\Delta \mu \neq \pi$. If the sextupole strengths are high, the dynamic aperture will thus decrease rapidly with $\delta$. Therefore it is ideal to design a bypass that requires a small nonlinear correction so as not to lose the beam. The location of the sextupoles is shown in Figure 5.9 with respect to the horizontal and vertical phase advances as well as to the dispersion. As seen in Figure 5.9, the phase does not advance significantly in the vertical plane, but in the horizontal plane a phase advance of $\approx \pi$ is achieved. Only the non-integer part of the phase advance is plotted here (in units of $\pi$ ); the seemingly discontinuous point 
sextupole kick, S1

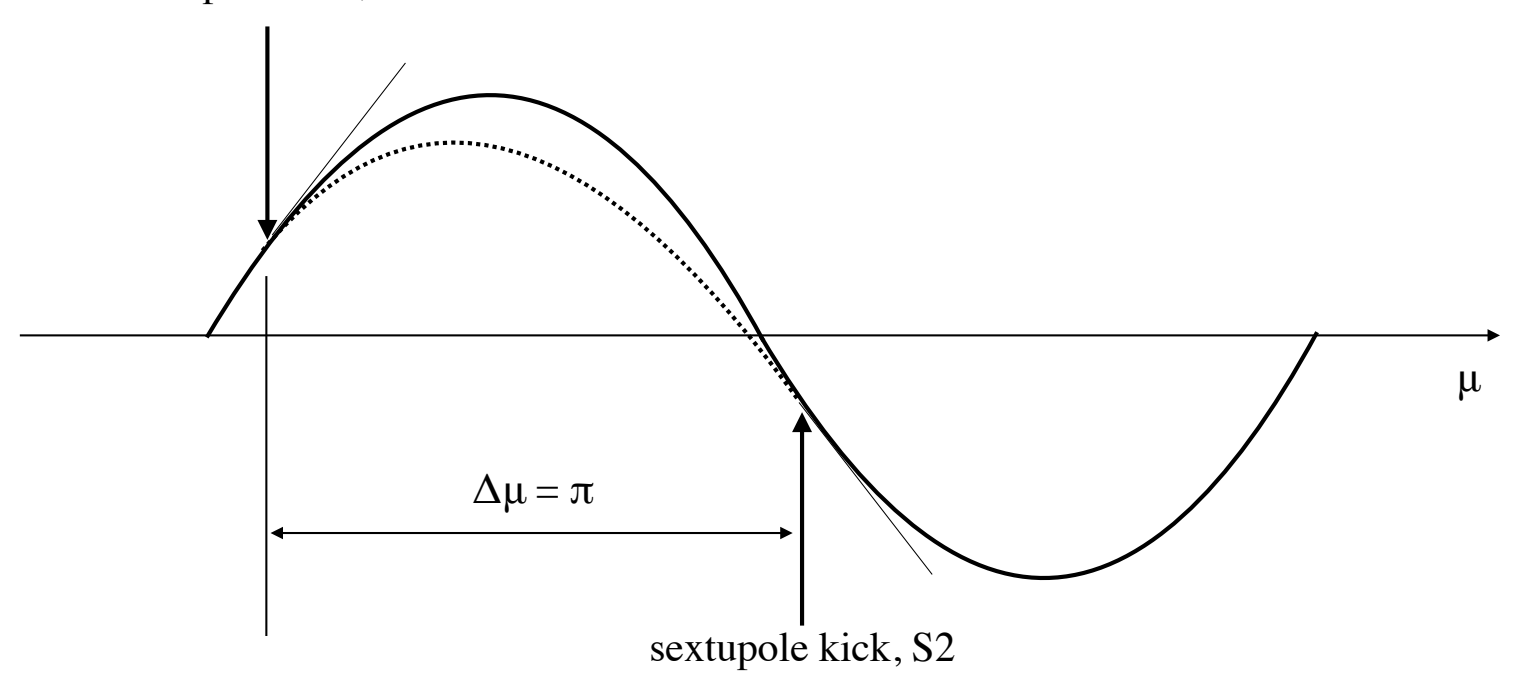

Figure 5.8. Two sextupole kicks separated by $\Delta \mu=\pi$ with the same polarity cancel each other's effect on the betatron oscillation.

at the middle of the diagram designates the point at which the phase advances past $\pi$. A geometrically proportional bypass is shown above the tune diagram to illustrate the value of the phase with respect to the location in the bypass.

If we assume the lengthening is due only to the horizontal emittance, we can make an estimate of the sextupole gradient needed to correct for this effect. First, we know the angle of the particle trajectory through the center quadrupole in terms of the horizontal emittance and beta function at that location: $\theta_{x}=\sqrt{\epsilon_{x} / \beta_{x}^{*}}$. Figure 5.10 shows the ray approximation in the bypass with $L_{q}$ as the focal length of the center quadrupole, and $L_{s}$ as the distance between the sextupoles and center of the bypass. The path length $\Delta L$ of a ray through the whole bypass can be estimated using a thin-lens approximation [29]:

$$
\Delta L=2 \frac{\theta^{2}}{2} L_{q}=\frac{\epsilon_{x}}{\beta^{*}} L_{q},
$$




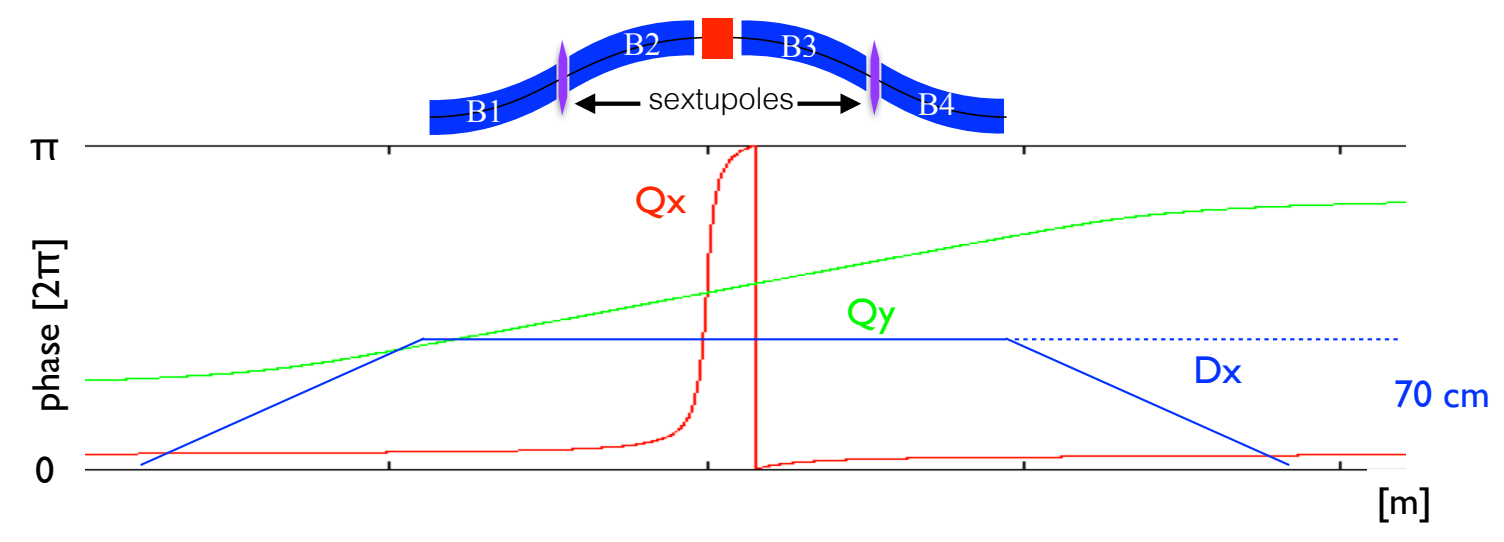

Figure 5.9. The horizontal (red) and vertical (green) phase advance along with the horizontal dispersion (blue) in the bypass

The angular correction supplied by the sextupoles separated by $\Delta \mu=\pi$ is given by

$$
\delta \theta=\frac{\rho s L}{p c} x^{2}=\frac{S L\left(L_{s} \theta\right)^{2}}{p c},
$$

where $S$ is the sextupole gradient and $\rho$ is the bending radius. If we require a path lengthening of $\Delta L_{s}=0.1 \mu \mathrm{m}$, we can solve for the only unknown variable, $S$, in the following equation:

$$
\Delta L_{s}=M_{52} \delta \theta=M_{52} \frac{\rho S L L_{s}^{2} \epsilon_{x}}{p c \beta^{*}},
$$

where $M_{52}$ is the matrix element that relates the angular spread to the path lengthening from $S 1$ to $S 2$, and $L_{s}$ is the distance between each sextupole and the middle of the chicane. It has been verified that the results from the MadX particle tracking agrees with this theoretical estimate, and corresponds to a field of $0.25 \mathrm{~T} / \mathrm{m}^{2}$ for each sextupole for a beam emittance of $1.6 \mu \mathrm{m}$.

\subsection{Optics Control in the Ring}

Once the linear optics in the bypass is set, we proceed to adjust the quadrupole gradients outside the bypass without moving preexisting magnets or rewiring circuits. In Figure 5.11, the dispersion invariant, $\mathcal{H}_{x}$ - as defined in Eq. 2.56 - is plotted (along with the horizontal beta and dispersion functions) around the ring starting and ending 


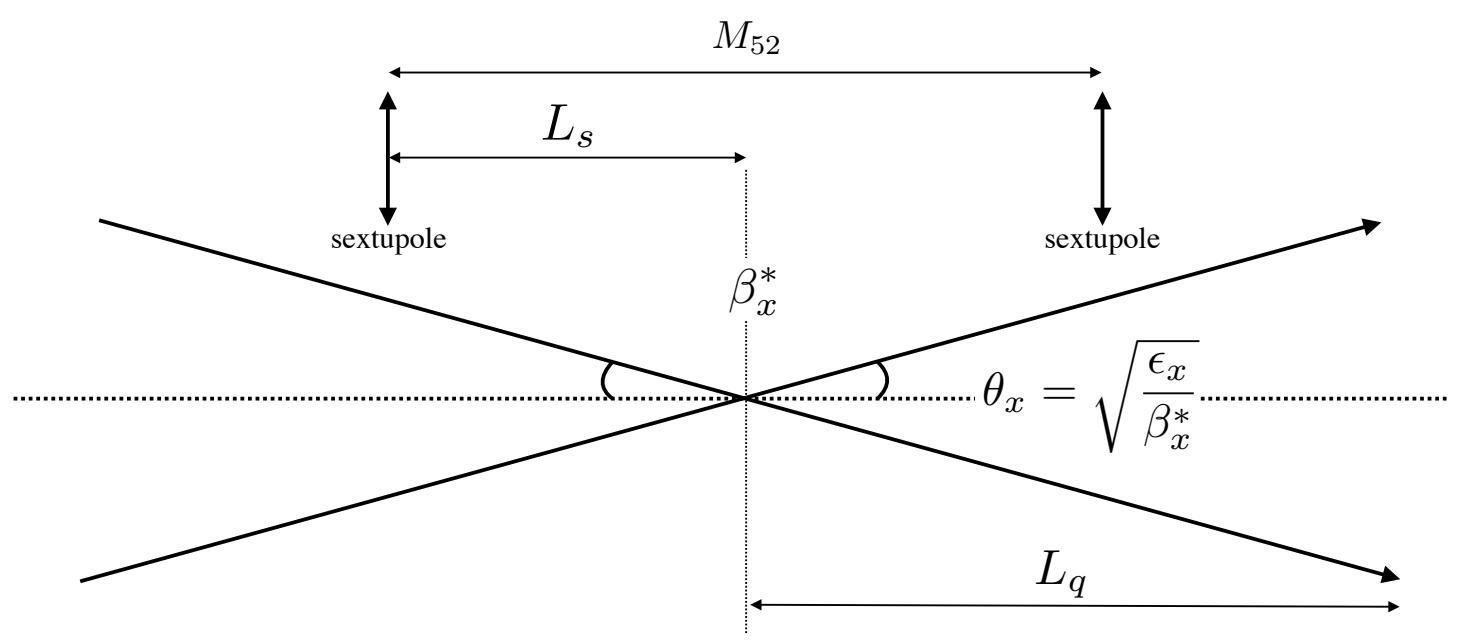

Figure 5.10. A first-order ray approximation for a trajectory with $\pi$ phase advance centered on the middle quadrupole in the bypass.

in the center of the bypass. The locations at which $\mathcal{H}_{x}$ changes (where $\frac{d \mathcal{H}_{x}}{d s}$ is nonzero) are coincident with the placement of the dipoles; in all other locations it remains constant. Since the equilibrium emittance, given in Equations 2.53, 2.55 and 2.56 is proportional to $\mathcal{H}_{x}$, the ring should be designed to minimize the dispersion invariant throughout the ring. To do this effectively, we adjust the optics in the ring so that $\mathcal{H}_{x}$ decreases in each of the dipoles until it is close to zero at the point in the ring opposite the bypass. Because the ring is symmetric, $\mathcal{H}$ will climb back up identically to how it declined after the midpoint.

Once we have done our best to minimize $\mathcal{H}$, the only other parameters that must be satisfied are the horizontal and vertical tunes, $Q_{x}$ and $Q_{y}$, respectively. We have chosen, as a working point, a tune that is between the half-integer and thirdorder resonance.

\subsection{Second Order Path Length}

We can estimate the path length through a bypass by taking into account first and second order terms. We assume there is no horizontal-vertical coupling in 


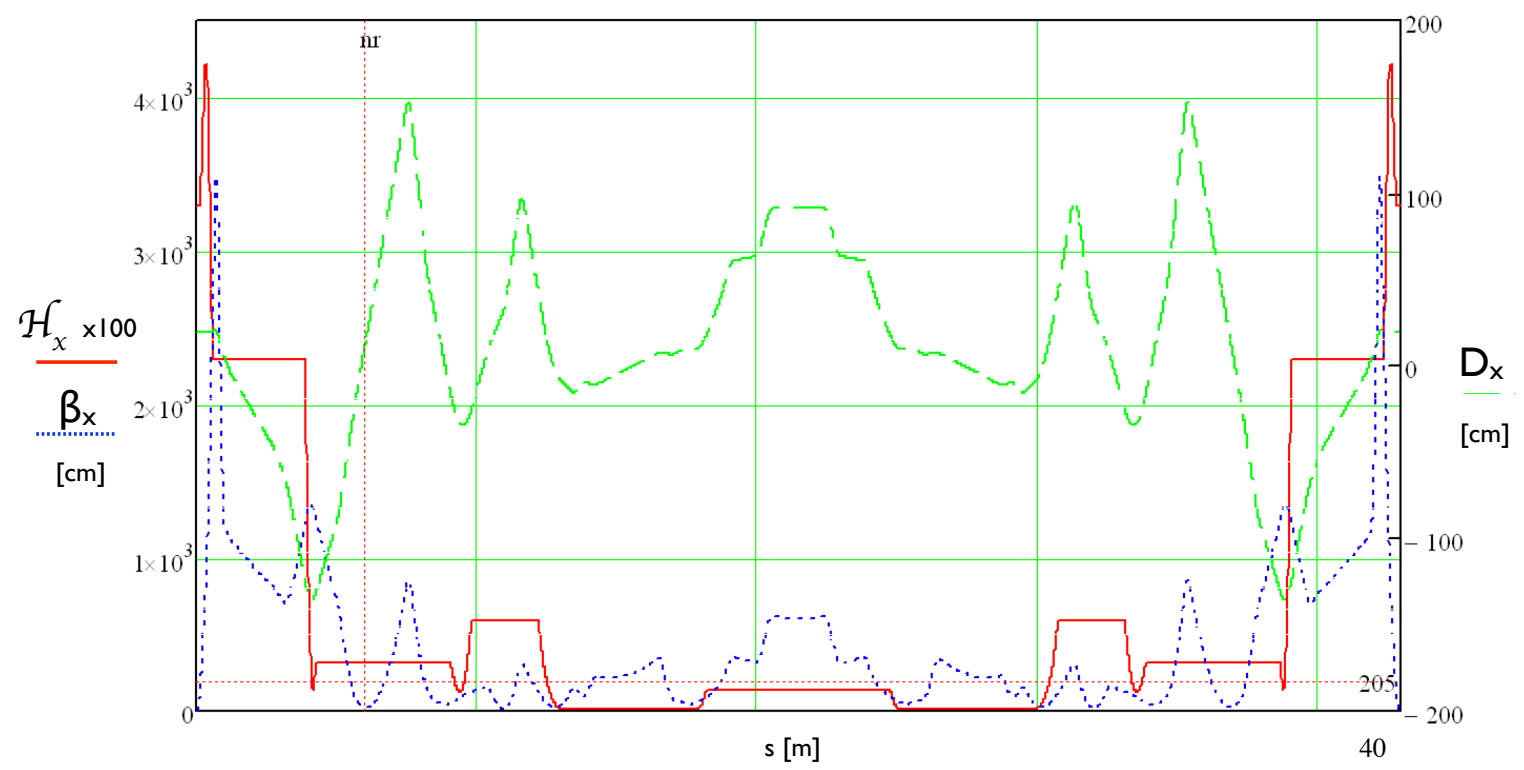

Figure 5.11. Dispersion invariant, $\mathcal{H}_{x}$, the dispersion, $D_{x}$, and the horizontal beta function $\beta_{x}$

the bypass, and little contribution from the vertical emittance to the path lengthening. Then, a vector being transported through the bypass is given by 4 first order coordinates and 10 second order coordinates, all of which are listed here:

$$
z=\left(x, x^{\prime}, l, \delta, x^{2}, x x^{\prime}, x l, x \delta, x^{\prime 2}, x^{\prime} l, x^{\prime} \delta, l^{2}, l \delta, \delta^{2}\right)^{T}
$$

where $l$ and $\delta$ are the longitudinal offset and momentum deviation. Following the steps outlined Section 5.3, the sample lengthening due to second-order effects (ignoring terms proportional to $l$ ) is [30]:

$$
\sigma_{z}^{2}=\epsilon_{x}^{2}\left[2\left(T_{511} \beta_{x}-T_{521} \alpha_{x}+T_{522} \gamma_{x}\right)^{2}+\left(T_{521}^{2}-4 T_{511} T_{522}\right)\right]
$$

where the matrix elements $T_{511}, T_{521}$ and $T_{522}$ are the second order matrix elements that are responsible for path lengthening. They are partially derived in Section 2.2 and are tabulated in Table 2.2. The second order contributions can easily be calculated using Equation 5.33. In the next section, we will attempt to minimize the path lengthening by placing sextupoles in the bypass to minimize one, or a combination, of these second-order matrix elements. 


\subsection{Sample Lengthening with the Polymorphic Tracking Code (PTC) Module in MadX}

To have the correct horizontally matched beam for the OSC bypass, we first need to know the Twiss parameters at the entrance of the bypass. These are provided by the MadX file translated from OptiM.

If the horizontal Twiss functions are known $\left(\beta_{x}, \alpha_{x}\right)$, a $2 \mathrm{D}$ beam with the appropriate $x$ - $x^{\prime}$ coordinates can be generated using a random multivariate module in Python with a specified covariance matrix defined in Equation 2.64. However, when considering a $4 \mathrm{D}$ beam that is horizontally and vertically coupled, another method of constructing a covariance matrix must be formulated. Using a model of the IOTA ring, a MadX tracking simulation was performed using the PTC particle tracking module. A matched beam was generated using the methods of the previous section and was tracked through the ring for 500 turns. In Figure 5.12, the beam distribution after 500 turns (red) is superimposed on top of the initial distribution (blue). Since the distribution is sampled at the same point in the ring, the blue and red distributions should have an identical shape and orientation. The congruence of the two distributions depicted in Figure 5.12 indicates that the beam used is properly matched to the ring. Further verification was done by calculating the Twiss parameters using the second moments of each distribution according to Equation 2.69 , and the relation

$$
\beta_{x}=\sigma_{x}^{2}-\left(D_{x} \delta\right)^{2} / \epsilon_{x}
$$

This $6 \mathrm{D}$ fully coupled beam was used to study path lengthening in the bypass. The bunch is roughly $1.1 \mathrm{~cm}$ long; it is tracked through the bypass, and each of the particles' 6D coordinates is observed at several places in the bypass. Figure 5.13 shows a histogram of longitudinal position of each particle in the bunch at the pickup, kicker and after each bending magnet in the bypass. The bottom figure is a plot of the 


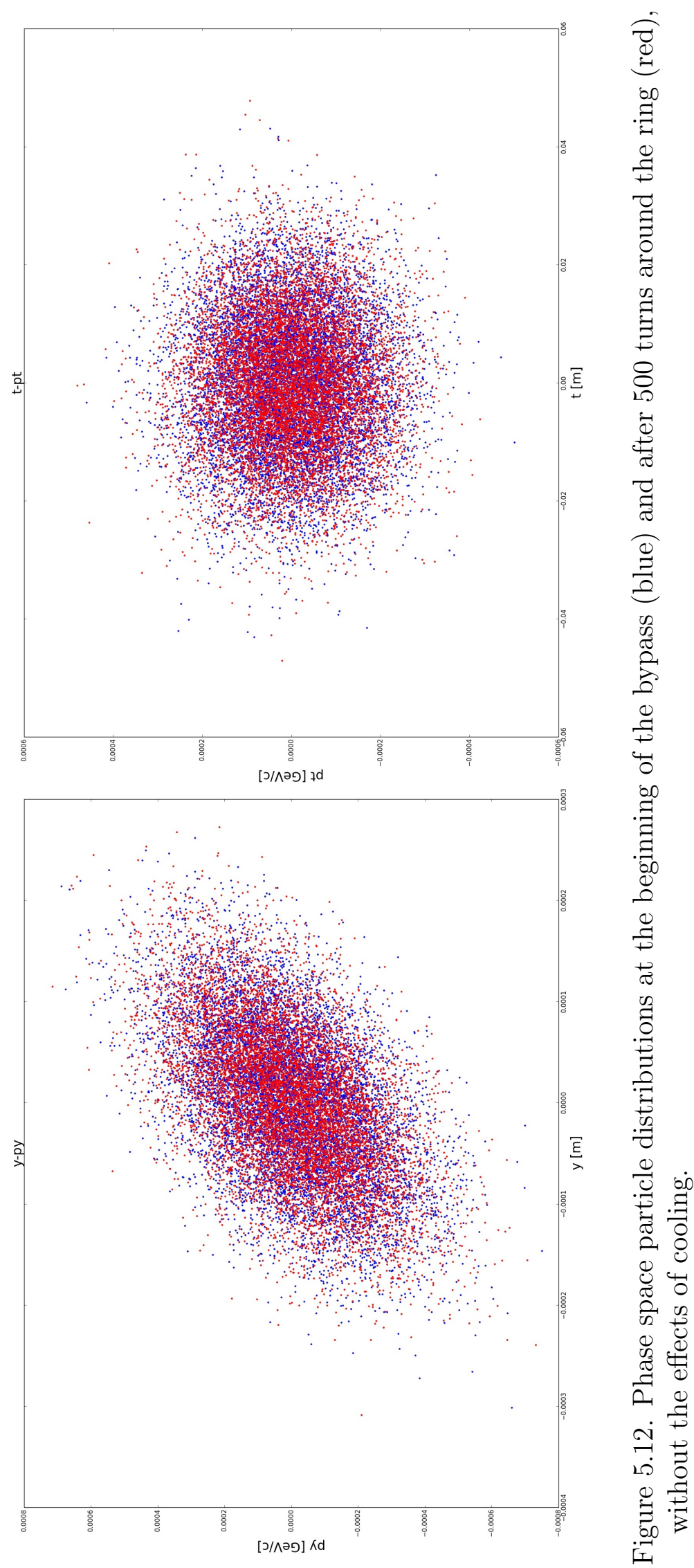


RMS values extracted from the top six plots. The histograms look identical, but the RMS values reveal that the bunch is actually being compressed by an amount that is several orders smaller than the over all bunch length. However, we are not interested in the absolute length of the bunch; rather, we want to measure the longitudinal separation of each particle from its initial position from the beginning to the end of the bypass with respect to the reference particle.

To find this value, we simply subtract the initial longitudinal position from the final for every particle $i$ :

$$
\Delta z_{i}=z_{i}^{k}-z_{i}^{p} .
$$

This allows us to start out with a delta function in $z$ and plot a histogram of the path length differences (with respect to the reference particle) through the bypass as seen in Figure 5.14. The bottom plots illustrate that the sample lengthening is generated only in the dipoles and is for the most part in agreement with the theory as seen in Figure 5.6 and replotted here in red. However, the effective RMS sample lengthening at the end of the bypass does not agree with theory (see Eq. 5.21). The discrepancy can be attributed to the skewed distribution. Equation 5.21 is only applicable to a Gaussian distribution, and in the last histogram, we can see that the distribution at the end of the bypass has a significantly long tail.

If we apply a cut to the tail of the distribution at $3 \sigma$, we regain closer correspondence with the theory. Several schemes were tried for sextupole corrections including adding higher order moments inside the bending magnets. However, the simple scheme of placing the sextupoles between bends 1 and 2 and between 3 and 4 proved to correct the lengthening the best. In addition, the length of the sextupoles did not affect the correction. The gaps between the dipoles are $6 \mathrm{~cm}$ long; all simulations presented here were carried out with $2 \mathrm{~cm}$ long sextupoles. The practicality of manufacturing such small sextupoles will be studied at a later time. Figure 5.15 


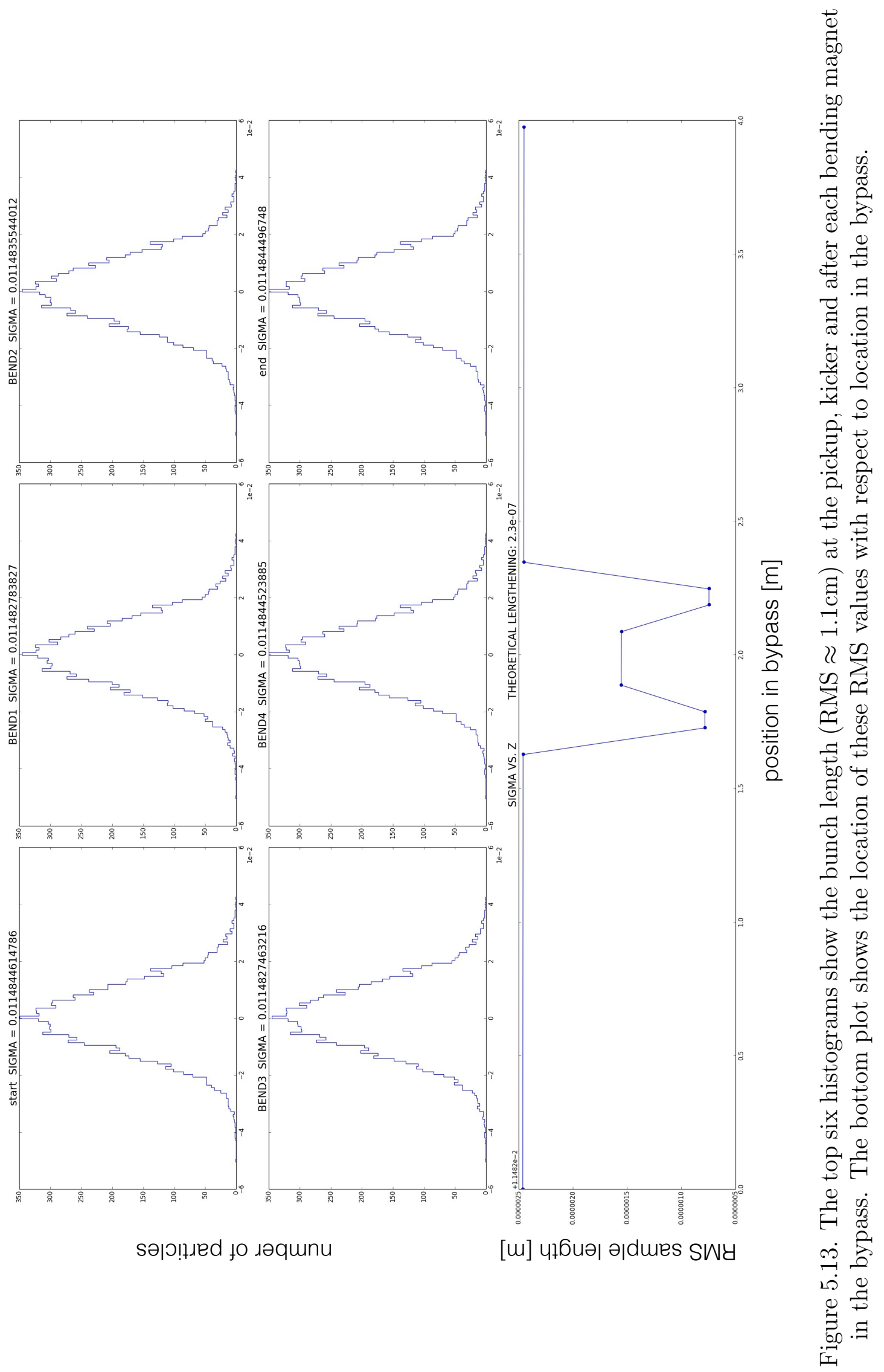




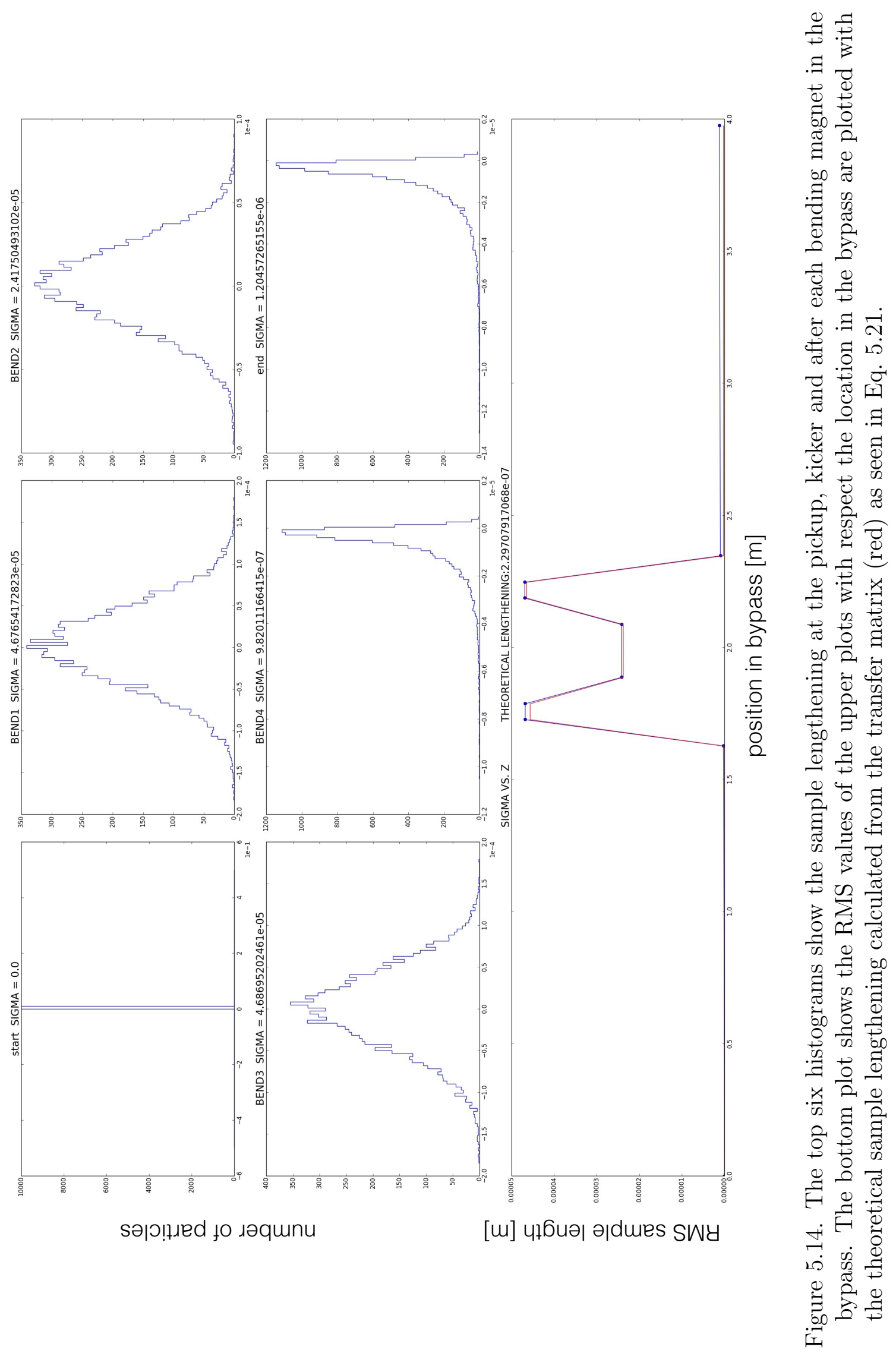


shows the result of the sextupole correction. The PTC module was scripted to minimize matrix element $T_{522}$ as it is the largest contributing factor to sample lengthening as seen in Table 2.2. This fitting script is documented in Appendix D. While it was possible to reduce the sample lengthening from $1.20 \mu \mathrm{m}$ down to $0.26 \mu \mathrm{m}$, it was impossible to reduce it below the required $0.2 \mu \mathrm{m}$ to fit inside the usable radiation wavelength. The next section will describe the needed initial beam that is required for the sextupole correction to produce an acceptable sample length.

To better understand the sextupole corrections in the bypass, a plot of the lengthening versus the particle's position in $x$ is plotted after each dipole without sextupole corrections (Figure 5.16), and with (Figure 5.17). After a 6D Gaussian beam is generated, narrow bands of particles located at 1, 2 and 3 sigma of the horizontal emittance were selected, and all other particles removed. A description of the process of generating this distribution, along with the Python code, is found in Appendix C. Only these bands were tracked though the bypass. The initial delta function centered at $z=0$ accounts for the identical subtraction scheme (Eq. 5.35) used in Figures 5.14 and 5.15. The parabolically shaped distributions in Figure 5.16 at the end of the bypass indicate the need for a second order correction. And as expected, when the sextupoles are added, the distribution flattens out as seen in Figure 5.17. The vertical spreads reflect the contribution of momentum spread to the lengthening of the samples at the end of the bypass, with faster particles displaced in the positive $z$ direction.

\subsection{Further Reducing the Sample Lengthening}

The goal of the OSC bypass optics is to keep the sample lengthening below the radiation wavelength $(0.2 \mu \mathrm{m})$. However with the scheme presented above, we were unable to reach that goal. Revisiting Equations 5.25 and 2.56, we can reduce the main contributor to sample lengthening, namely the horizontal emittance, by 


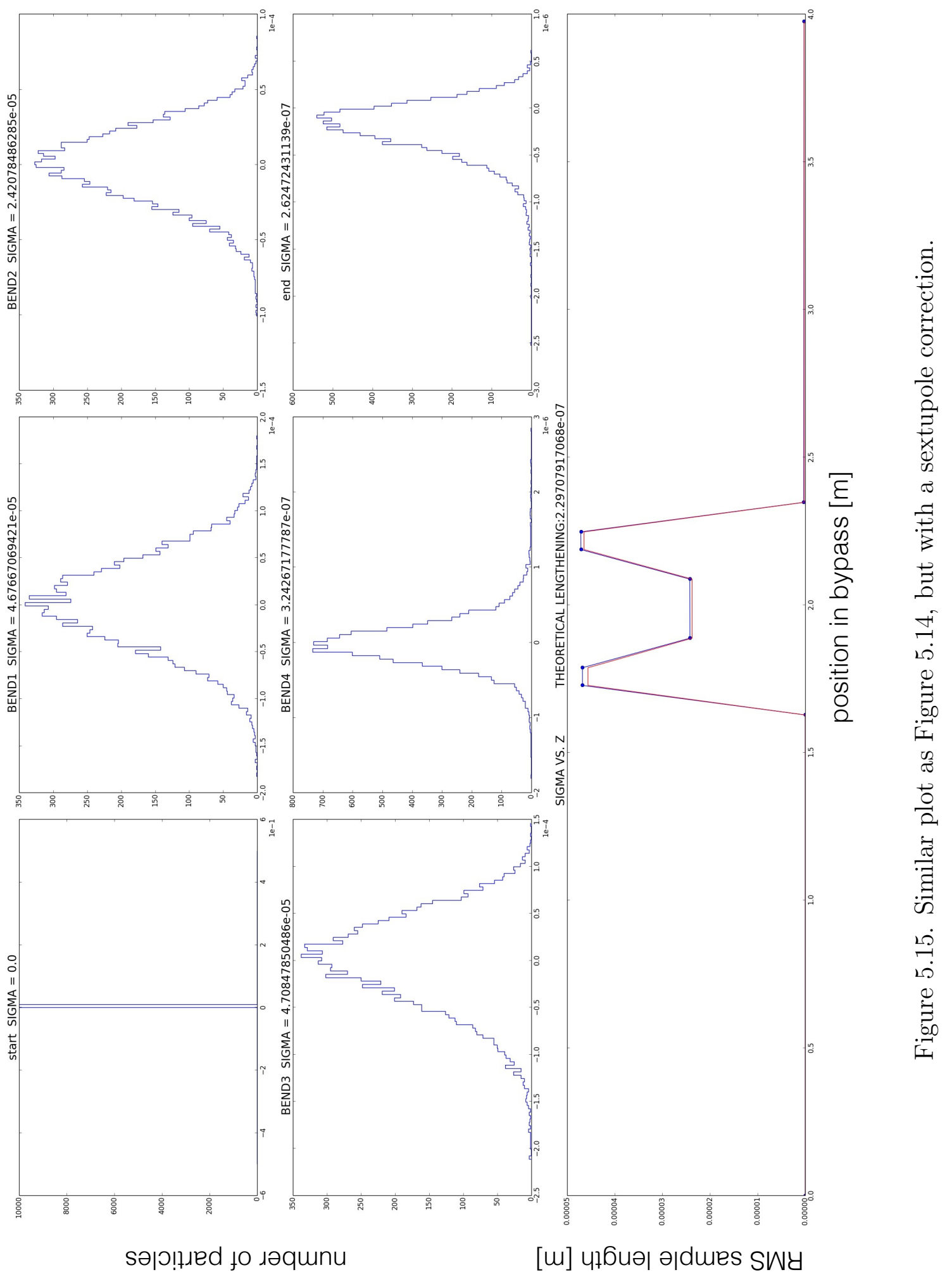



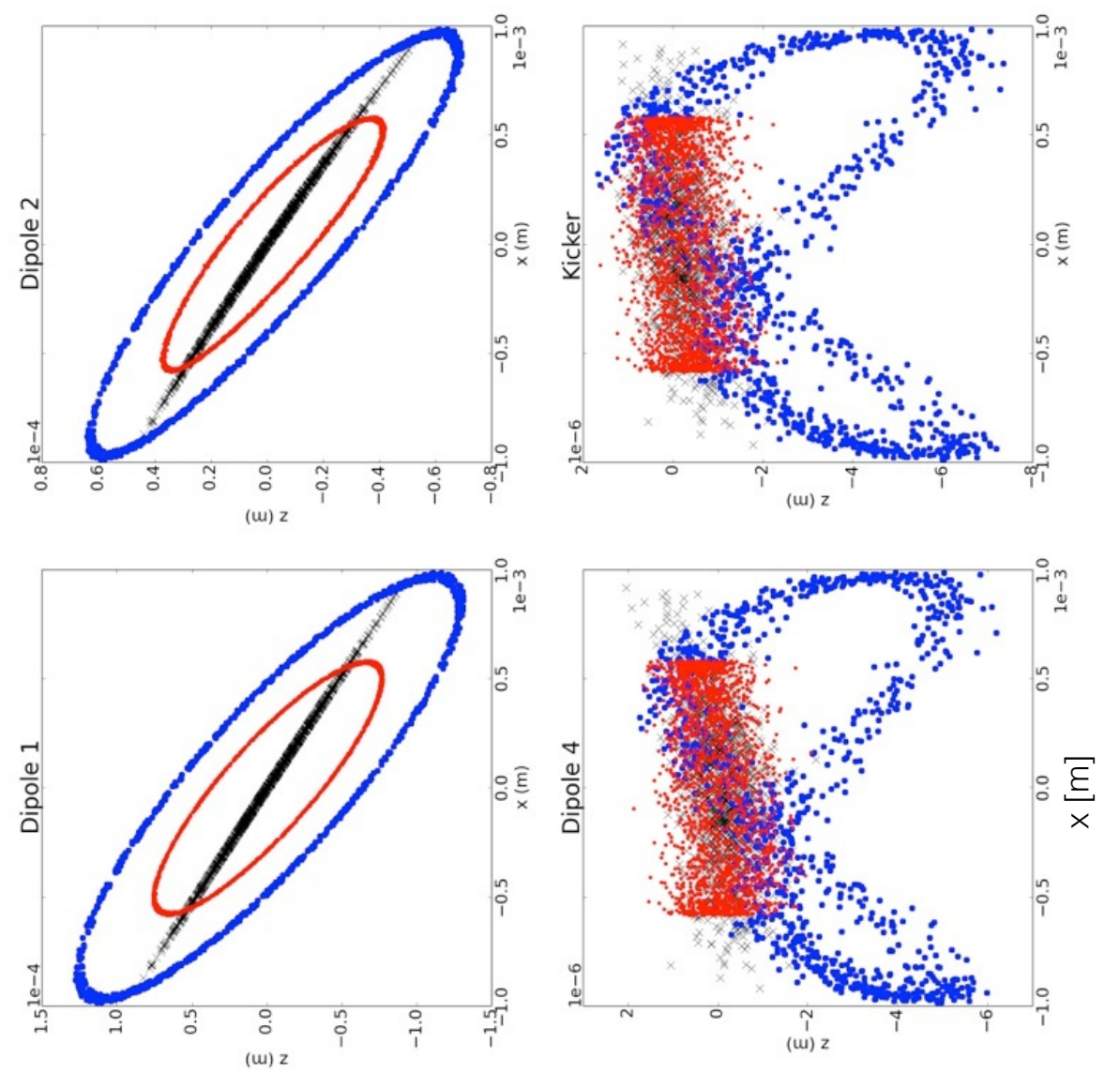

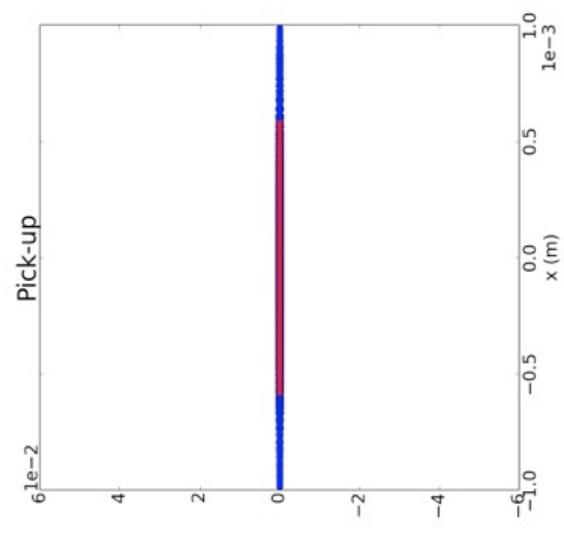

[w] z

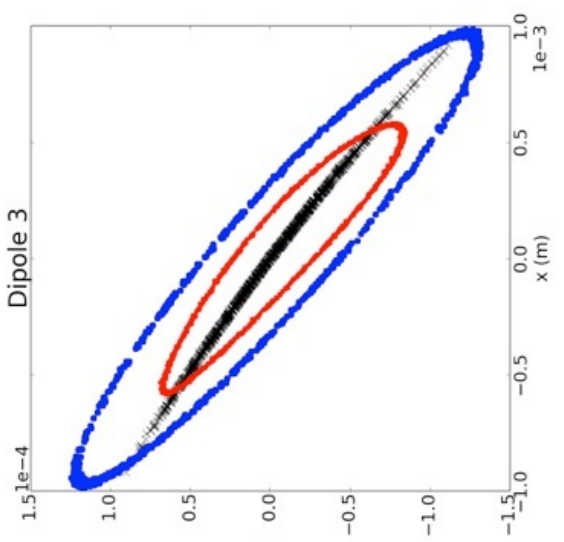

[w] z

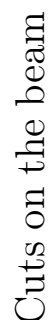

过

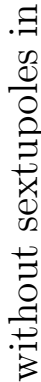

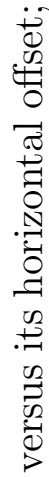

룰

㐘

تf

苂

.

으일

告

䒕饮

2.

要

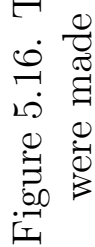



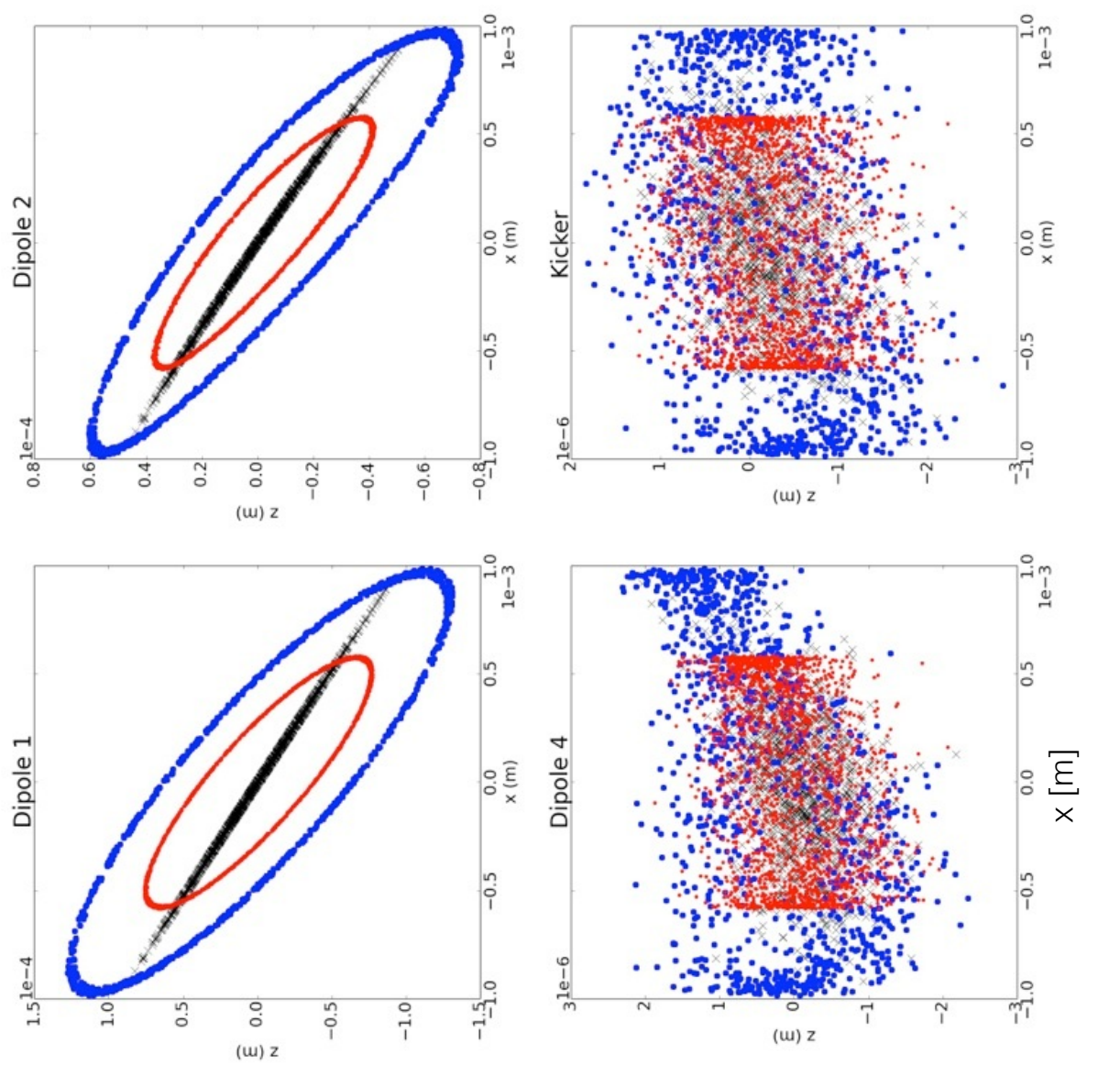

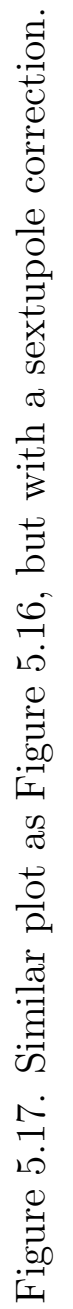
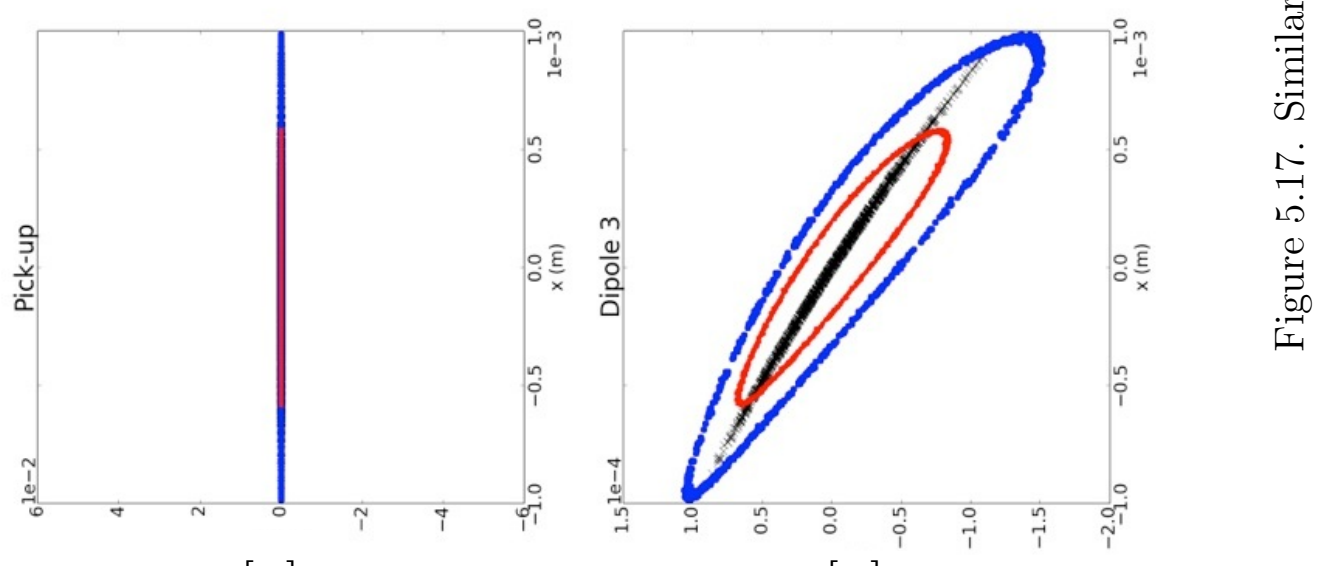

[w] z

[w] z 
decreasing the dispersion in the center of the bypass. This can be done without compromising the damping rates (Equation 5.25) by increasing the horizontal offset in the center of the chicane. This is done by lengthening the dipoles. Several dipole lengths were tried and the dispersion was reduced in the bypass accordingly. This in turn affected the horizontal emittance used, as described in Equation. 2.56. A matched beam was then tracked through the bypass with and without sextupole correction and the RMS sample lengthening obtained. The RMS sample length at the exit of the bypass is plotted versus the normalized emittance in Figure 5.18. The various dipole lengths used in the bypass (10, 12, 14, 16 and $18 \mathrm{~cm}$ )-affecting only the horizontal displacement, but not the delay - display a clear linear correlation in sample lengthening (along with the lengthening corrected via sextupole correction) with the equilibrium emittance of the ring.

In order to reduce the sample lengthening below $0.2 \mu \mathrm{m}$, it is desirable to have a horizontal emittance of $\approx 1.2 \mathrm{~nm}$. This can be done by reducing the horizontal dispersion in the bypass; and to conserve the damping decrements, the dipoles must be lengthened in order to increase the horizontal offset. A sufficiently low dispersion and large enough horizontal offset can be achieved with $18 \mathrm{~cm}$ long dipoles (see Figure 5.18). By further lengthening the dipoles and decreasing the dispersion in the bypass it is foreseeable that the sample lengthening can be suppressed even more; however, as discussed in the next section, it is beneficial to not reduce the emittance more than is required. The same studies were conducted with this configuration and similar plots show the uncorrected and corrected sample lengthening: Figures 5.19 and 5.20 respectively. The same $x-z$ plots were made to show the correction more explicitly in Figures 5.21 and 5.22. The parameters of the OSC bypass are summarized in Table 5.1 for both configurations. It is clear from Figure 5.19 that the beam distribution exhibits a more distinct second-order distribution at the end of 


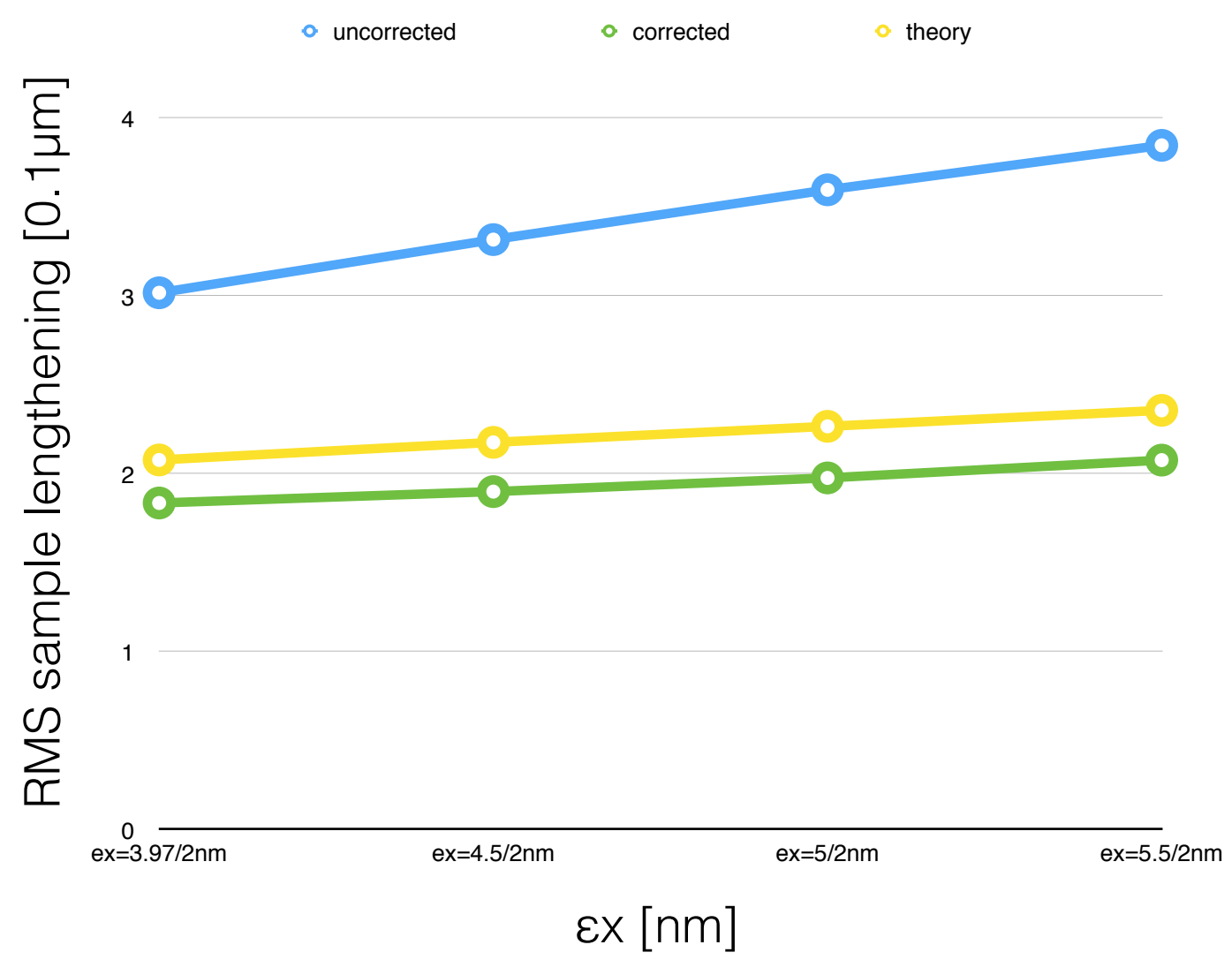

Figure 5.18. The horizontal emittances were varied using several bypass configurations. The uncorrected and corrected RMS sample lengthening (blue and green respectively) are plotted versus the horizontal emittance and compared to the theoretical sample lengthening (yellow).

the bypass than Figure 5.16. A sextupole correction is therefore more effective when applied to the distribution.

The bypass designed with $\frac{D^{*}}{\beta^{*}}=\frac{30 \mathrm{~cm}}{0.8 \mathrm{~cm}}$ required a sextupole strength of 0.25 $\mathrm{T} / \mathrm{m}^{2}$ to correct the path length, whereas the longer bypass designed with $\frac{D^{*}}{\beta^{*}}=\frac{15 \mathrm{~cm}}{1.4 \mathrm{~cm}}$ required a sextupole strength of $0.01 \mathrm{~T} / \mathrm{m}^{2}$ to correct the path length. In the first design, not only was the path length not fully corrected $(\approx 40 \%$ too long), but the strong sextupole gradients severely degraded the dynamic aperture. The dynamic aperture was calculated by A. Valishev using an in-house 6D particle tracking code. 


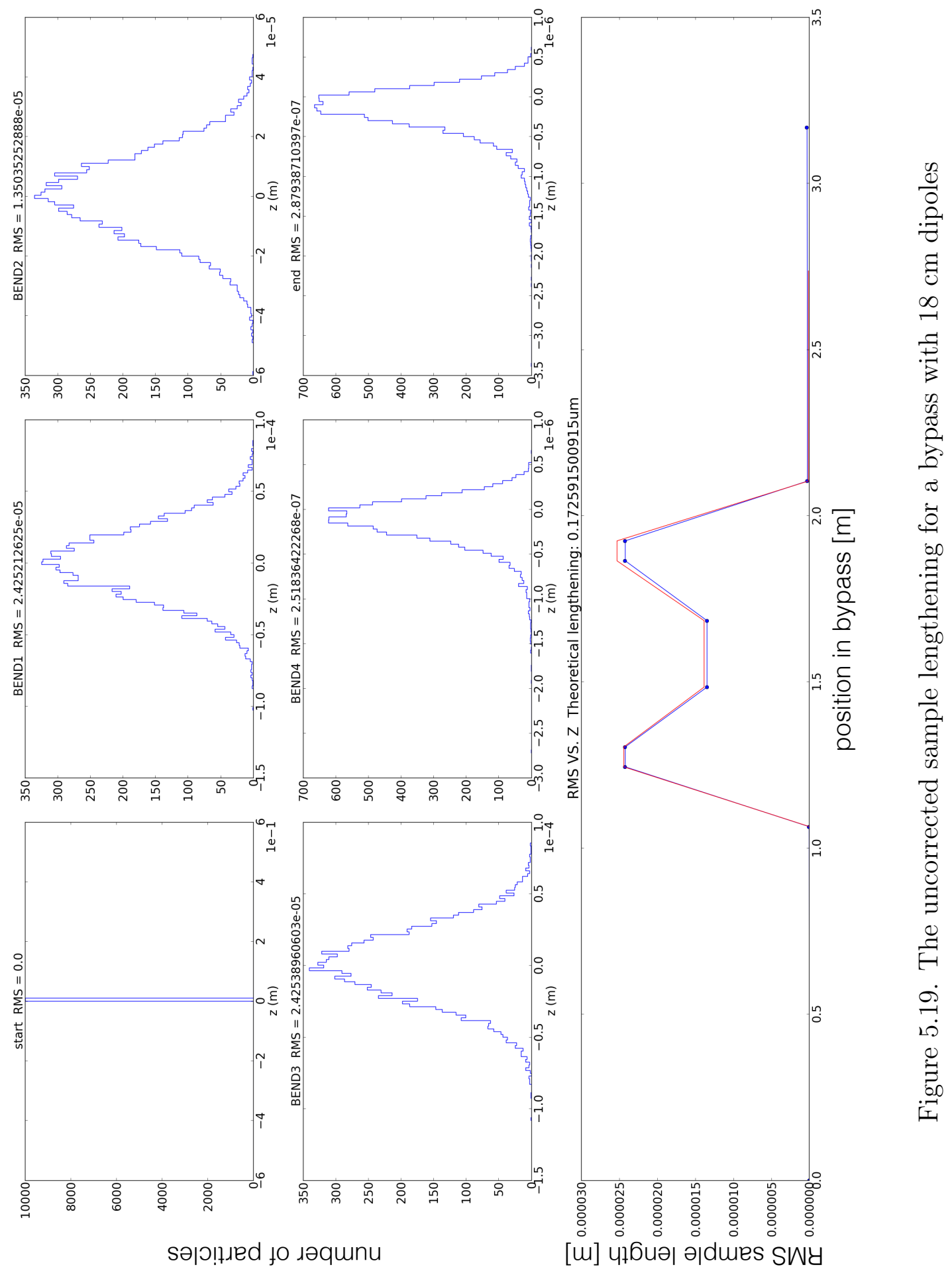



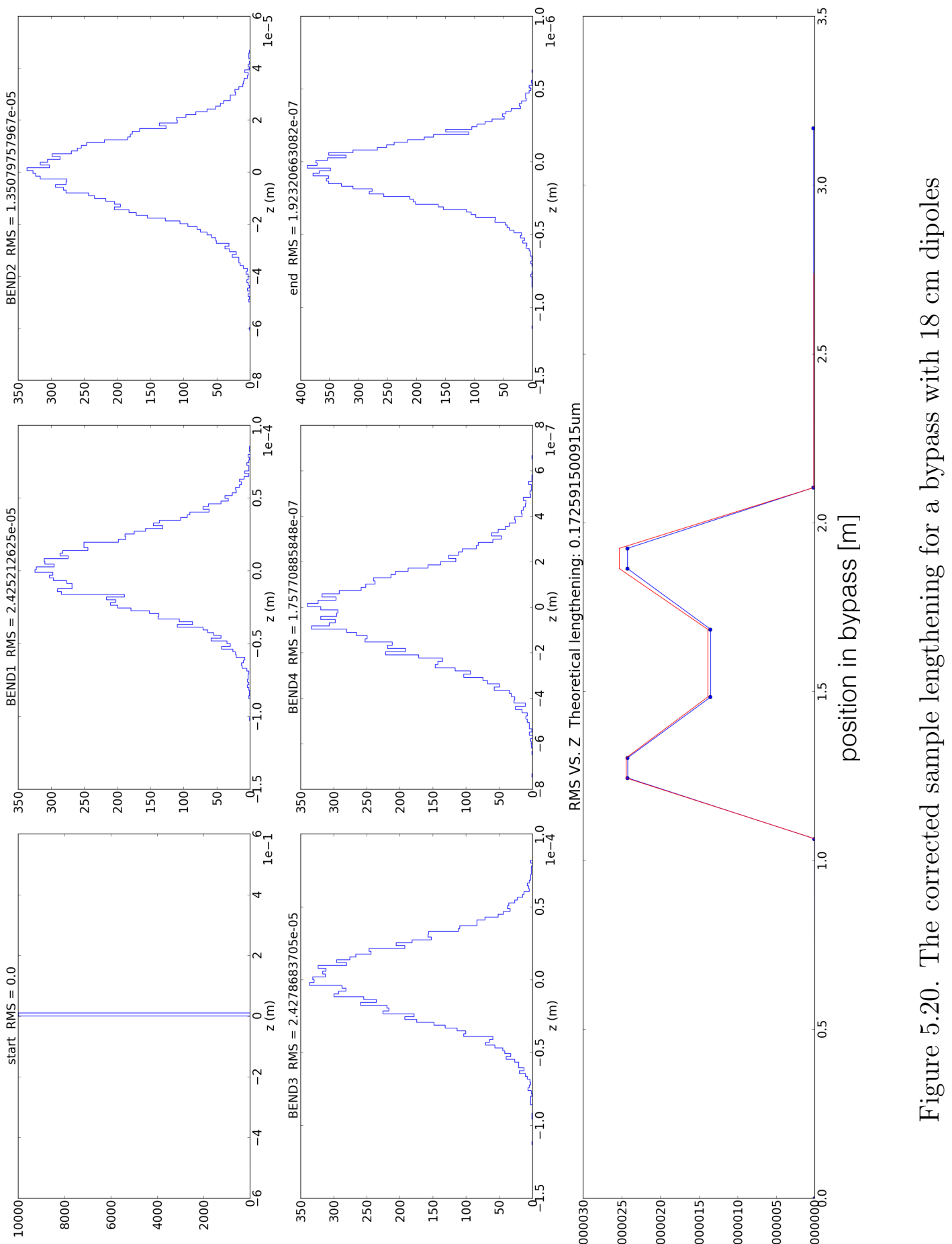

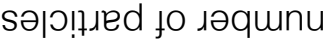

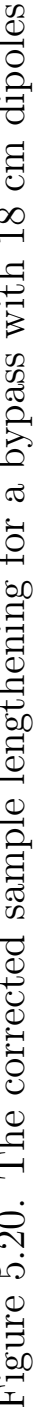

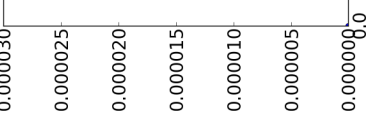

[w] ułচuə| ә|dues Swy 
Table 5.1. OSC Beam Parameters

\begin{tabular}{cccc}
\hline \hline Parameter & Short Dipoles & Long Dipoles & Units \\
\hline Energy $E_{0}$ & 100 & - & $\mathrm{MeV}$ \\
RMS momentum spread & $1.11 \times 10^{-4}$ & $1.11 \times 10^{-4}$ & - \\
Transverse RMS emittances, $\epsilon_{x}=\epsilon_{y}$ & 11.5 & 1.23 & $\mathrm{~nm}$ \\
RF Harmonic; Voltage & $4 ; 100$ & $4 ; 100$ & $-; \mathrm{V}$ \\
Dipole: magnetic field $\times$ length & $4.22 \times 18$ & $1.32 \times 10$ & $\mathrm{kG} \times \mathrm{cm}$ \\
Integraded strength of central quad, $G d L$ & 1.58 & 0.355 & $\mathrm{kG}$ \\
Delay in the bypass, $\Delta s$ & 2 & 3 & $\mathrm{~mm}$ \\
Horizontal beam offset, $h$ & 2.01 & 3.74 & $\mathrm{~cm}$ \\
$M_{56}$ & 3.95 & 4.92 & $\mathrm{~mm}$ \\
$D^{*} / \beta^{*}$ & $30 / 0.8$ & $15 / 1.4$ & $\mathrm{~cm}$ \\
Cooling rates ratio, $\lambda_{x}=\lambda_{y} / \lambda_{s}$ & 1.18 & 3.851 & - \\
Cooling Ranges: $N \sigma_{p} / N \sigma_{x}$ & $2.1 / 3.2$ & $6.2 / 4.8$ & - \\
Bunch Length & 18 & 18 & $\mathrm{~cm}$ \\
Sextupole Strength & 0.25 & 0.01 & $\mathrm{~T} / \mathrm{m}^{2}$ \\
Sample Lengthening Without Correction & 1.6 & 0.32 & $\mu \mathrm{m}$ \\
Sample Lengthening With Correction & 0.27 & 0.197 & $\mu \mathrm{m}$ \\
\hline
\end{tabular}

The final iteration of the OSC bypass as outlined in this section does not degrade the dynamic aperture nearly as much and it is foreseeable that using sextupoles in the ring will restore most of what is lost of the aperture. The parameters for each design of the chicane are tabulated in Table 5.1.

\subsection{Further Considerations for OSC optics}

The effect of IBS is amplified for bunches with small emittances. The small horizontal emittance required for the path lengthening to be under $0.2 \mu \mathrm{m}$ requires a beam with only 750,000 particles per bunch for the effects of IBS as seen in Equation 2.58 to remain controlled - a reduction of about half from previous bypass designs. For such a small beam current, very sensitive BPMs must be used to monitor the beam. Further investigation of this constraint is required. 

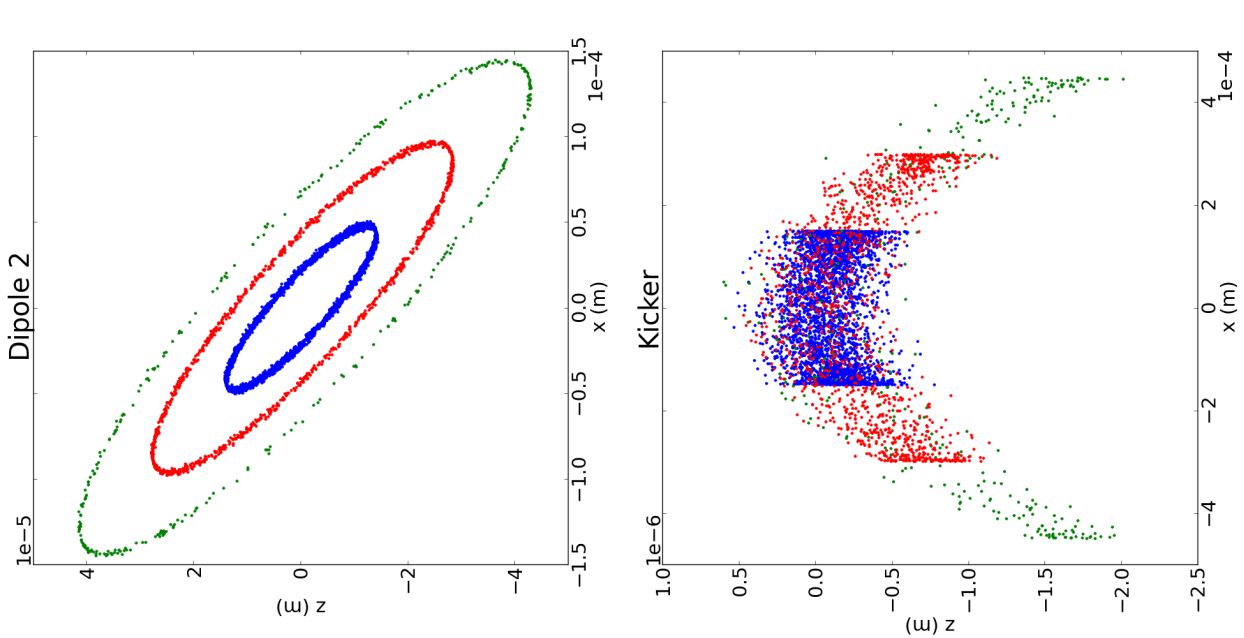

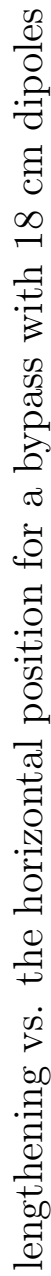
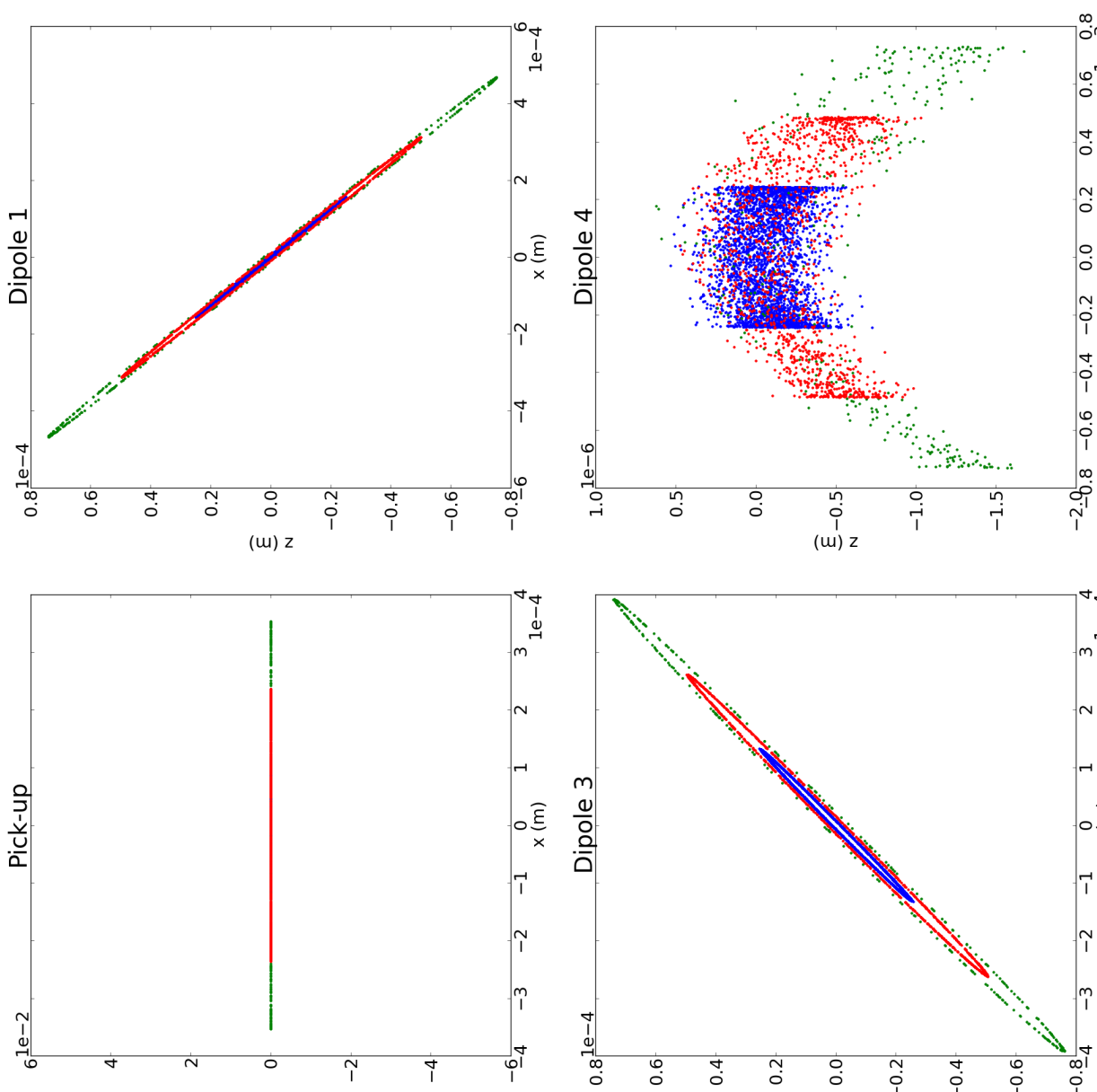

[w] z

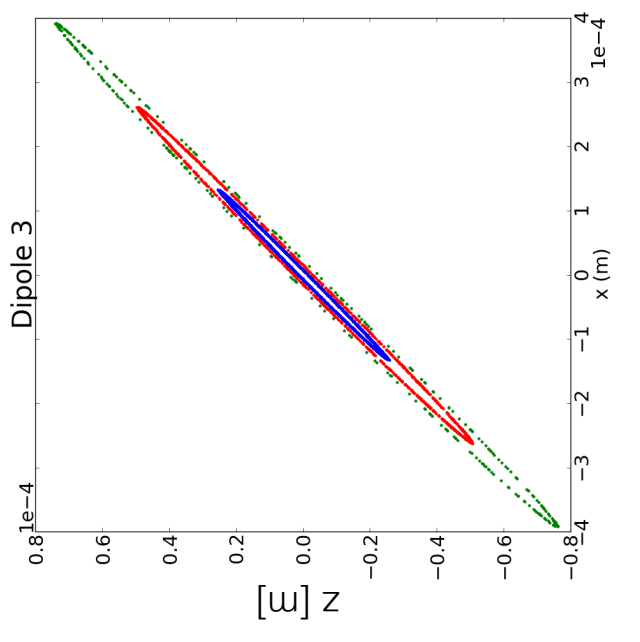

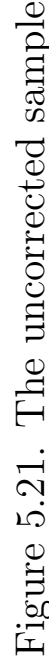



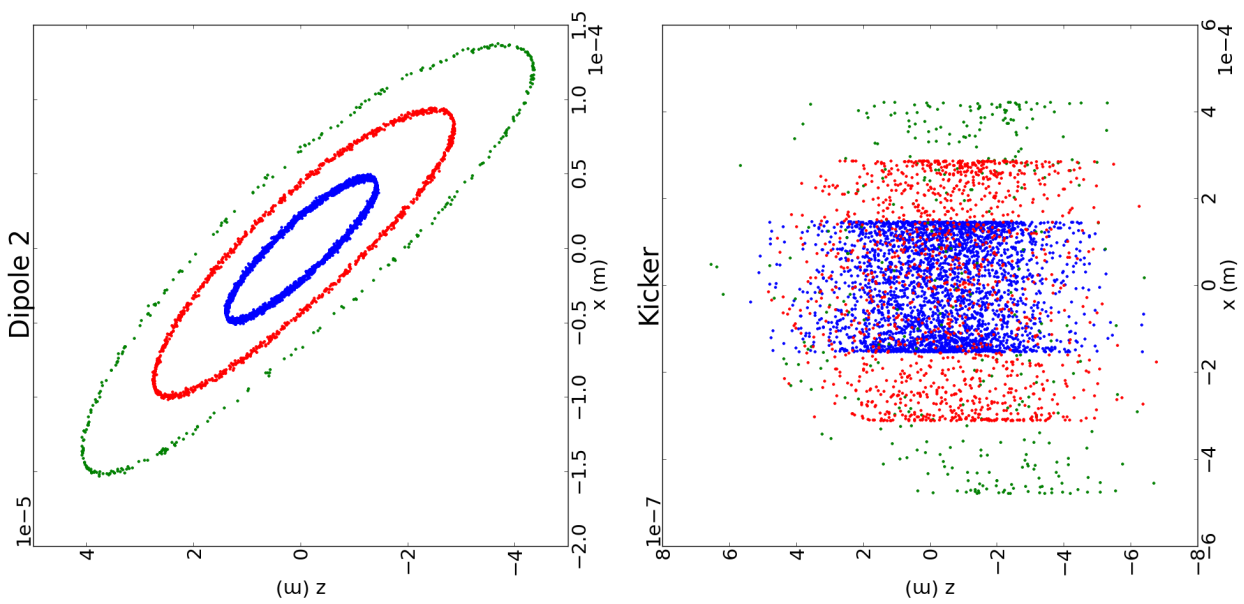

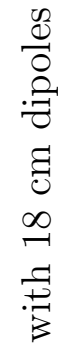
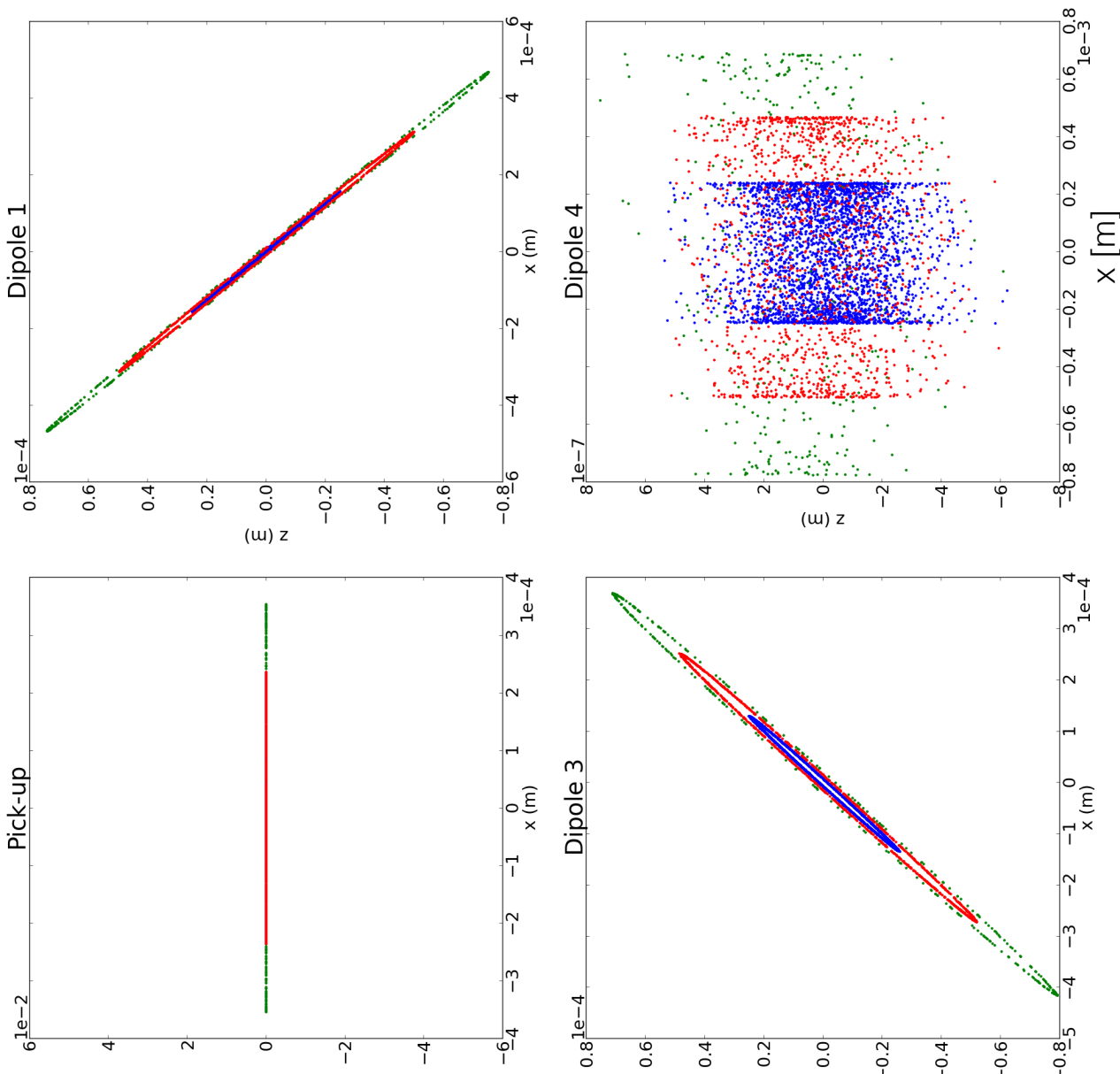

[w] z

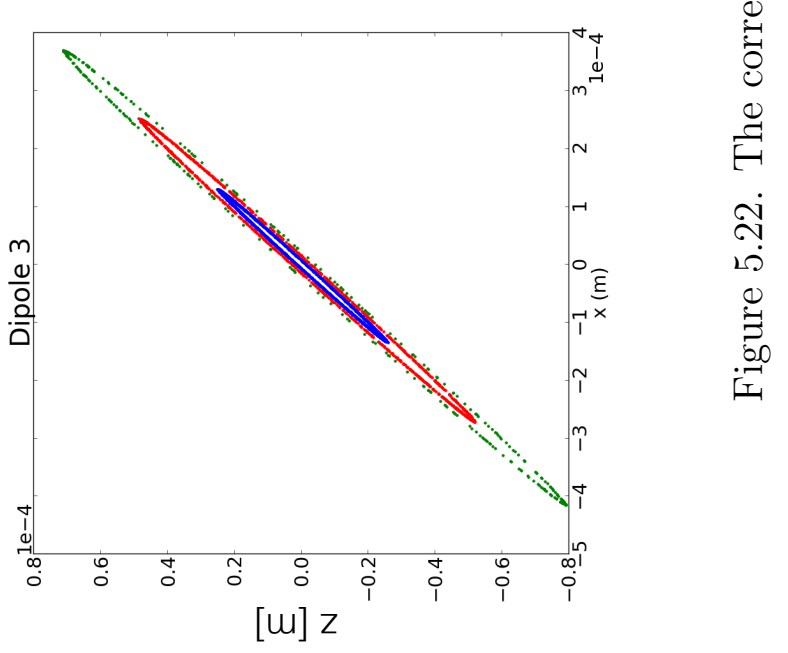


Misalignment and gradient errors in the bypass were not considered in the path lengthening studies described above. A similar study that takes these errors into consideration must be carried out in a similar manner as in [31].

\subsection{OSC Simulation Studies}

As a proof of principle, a simplistic simulation of OSC cooling was carried out using MadX and the tracking module in PTC. A Python script is needed to track particles and calculate and apply longitudinal kicks (the script is documented in Appendix E). There are several step to this simulation: first, a single particle (with the appropriate 6D coordinates) is generated and tracked through the bypass; then, at the end of the bypass, a Python script records the longitudinal offset of the particle and calculates a kick $(\Delta E)$ given by:

$$
\Delta E=\delta E_{\max } k \Delta s
$$

where $\delta E_{\max }=50 \mathrm{eV}$, and radiation wave number $k=\frac{2 \pi}{0.8 \mu \mathrm{m}}$ [29]. The magnitude of the kick $\delta E_{\max }$ if made large enough will damp the longitudinal momentum after one pass through the bypass; too high, and it will heat the longitudinal phase space. Each of the correcting kicks applied to a single particle on every consecutive revolution around the ring is recorded and displayed in Figure 5.23. After the particle makes one pass through the bypass, the script then rewrites the input file with the calculated kick and runs the simulation again, this time with the applied kick. After the kick is applied, the particle makes a revolution around the rest of the ring. For a momentum spread of $1.11 \times 10^{-4}$, it is estimated that the bunch will be damped after $N=$

$\frac{10^{8} * 1.11 \times 10^{-4} \mathrm{eV}}{50 \mathrm{eV}}=260$ turns. Therefore, this process is iterated $\approx 300$ times before a second particle can be tracked in a similar manner. A particle executing synchrobetatron oscillations around the ring will trace out a closed circle in the longitudinal phase space $z-p_{z}$; and if it is kicked longitudinally based on its momentum, on each 


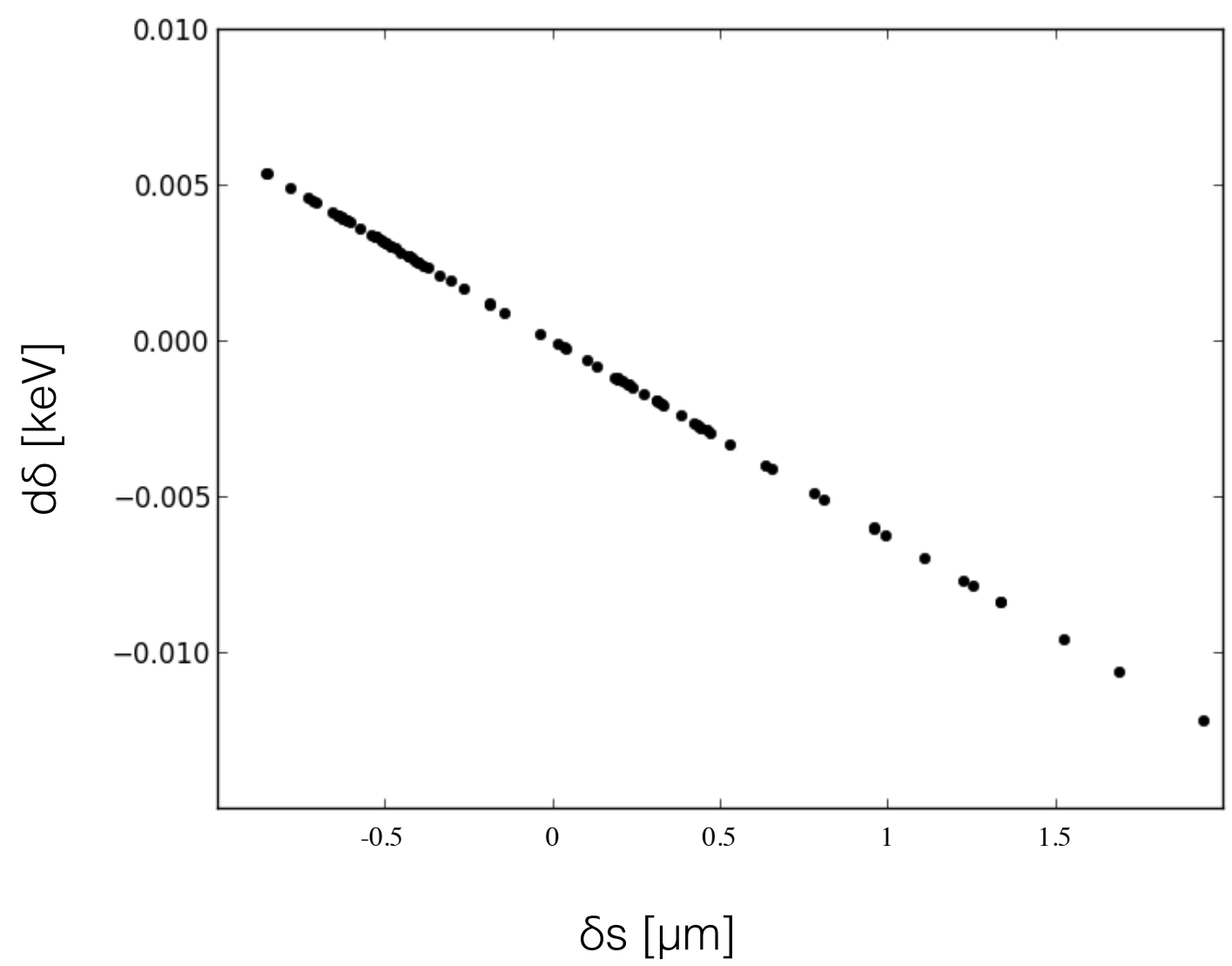

Figure 5.23. The magnitude of each kick with respect to the particle's longitudinal offset on each revolution around the ring.

turn instead of tracing out a circle, it will spiral inward to occupy a smaller segment of the phase space. An example of this motion is shown in Figure 5.24.

\subsection{OSC Summary}

The design of the OSC bypass as laid out in this chapter successfully suppresses the sample lengthening from pickup to kicker while allowing for sufficiently large cooling ranges of 6.2 and 4.8 in the horizontal and vertical planes respectively. The factor $\Phi D^{*} h$ (see Equation 5.25) determines the cooling dynamics. By reducing the dispersion in the center of the bypass, $D^{*}$, and increasing the horizontal offset, $h$, we are able to minimize the equilibrium emittance in the ring without compromising 


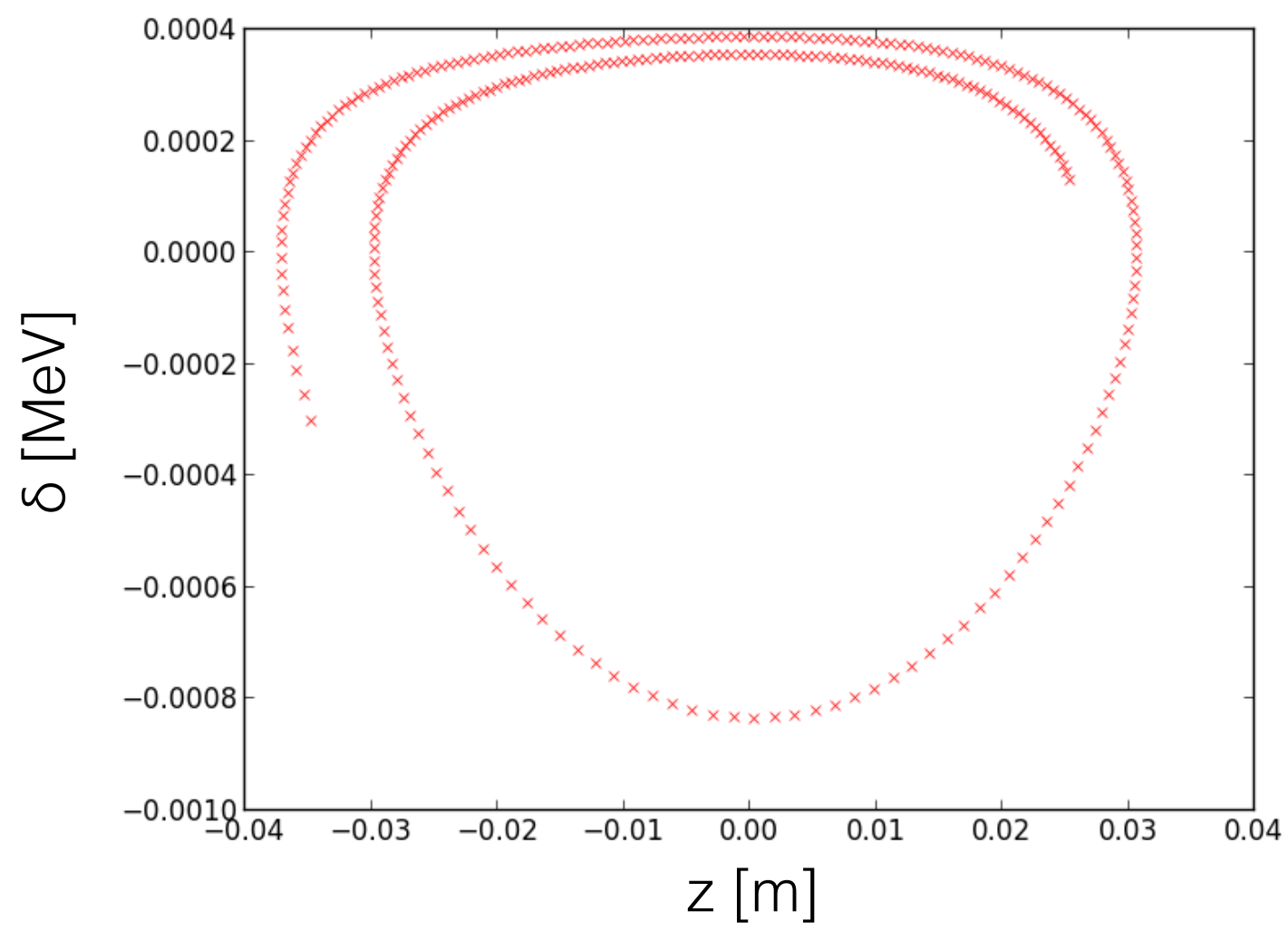

Figure 5.24. A particle's coordinates in $z-\delta$ phase space as it is tracked turn-by-turn in a storage ring due to synchrotron radiation and $\mathrm{RF}$ acceleration. Here the particle spirals inward as it is kicked on each consecutive turn. The asymmetry for $\delta>0$ compared to $\delta<0$ can be attributed to kicks not being centered on a $z$ offset of zero.

the cooling dynamics. The sextupole gradients needed to correct a sample within a bunch with emittance $\epsilon_{x} \approx 1.2 \mathrm{~nm}$ are 25 times smaller than the gradients required to correct a sample within a bunch with emittance $\epsilon_{x} \approx 12 \mathrm{~nm}$.

A simulation showing the damping of a single particle with the parameters described above has been carried out in MadX. 


\section{CHAPTER 6}

\section{CONCLUSION}

\subsection{IOTA Lattice}

The linear lattice of the IOTA ring has been designed to meet all the requirements for the nonlinear magnet experiments, the electron lens experiment and the OSC experiment. Some of these requirements are:

1. proper betatron phase advances inside and outside the nonlinear inserts,

2. equal horizontal and vertical beta functions at the entrance and exit of each nonlinear insert,

3. low beta functions throughout the ring in both planes,

4. zero horizontal dispersion in the nonlinear inserts, and

5. stability in all three planes.

It has been optimized to be cost effective and to retain as much of its layout as possible from one experiment to the next. The current layout makes use of most of the experiment hall leaving just enough room for forklifts around the perimeter.

Because the physical aperture of the undulators in the OSC experiment is too small for the integrable optics experiments, the OSC experiment will have to be installed and run after the completion of the integrable optics experiments.

The final layout of the IOTA ring (without the OSC insert) comprises 39 quadrupoles and 8 sector dipoles. BPMs and corrector packages have been placed to ensure acceptable beta function and phase advance errors [20].

6.1.1 Future Work. The placement of sextupoles must be considered in order to correct of the chromaticity of the ring. An obvious placement is close to the triplet 
between the $30^{\circ}$ bends. Once placed, a study of the dynamic aperture must be carried out.

\subsection{OSC Experiment}

It has been seen that with two sextupole correctors in the bypass, and with a small enough emittance $\left(\epsilon_{x}=\epsilon_{y} \approx 1.2 \mathrm{~nm}\right)$, the OSC experiment may be feasible in the IOTA ring.

Previous sextupole corrections on a beam with a larger emittance required large sextupole gradients. These strong fields were not able to sufficiently reduce the sample lengthening to fit into the usable segment of the radiation wavelength and in addition significantly degraded the dynamic aperture.

We have chosen the usable portion of the radiation wavelength $\left(\lambda_{R}\right)$ to be 0.2 $\mu \mathrm{m}$ for the OSC experiment meaning the RMS sample length must not exceed this length from the pickup to the kicker. With a sextupole correction it has been shown that a solution exists.

The quantity $\Phi D^{*} h$ determines the cooling dynamics in the OSC experiment. To reduce the emittance in the ring, the dispersion $D^{*}$ in the bypass center was reduced. The horizontal offset, $h$, was increased by lengthening the dipoles in the bypass in order to retain sufficient damping rates and cooling ranges. After these changes, the dispersion invariant was reduced in the whole ring to obtain the smallest emittance possible. With these corrections we were able to

1. reduce the emittance from $12 \mathrm{~nm}$ to $1.2 \mathrm{~nm}$ in the horizontal and vertical planes,

2. obtain sample lengthening less than $\lambda_{R}$,

3. retain good damping rates $\left(\lambda_{x} / \lambda_{s}=\lambda_{y} / \lambda_{s}=3.85\right)$,

4. retain good cooling ranges $\left(N \sigma_{p} ; N \sigma_{x}=6.2 ; 4.8\right)$, and

5. retain good dynamic aperture by utilizing low sextupole gradients. 
6.2.1 Future Work. A more detailed simulation with realistic magnetic fields and errors is needed to ensure that the sextupoles can correct for the sample lengthening despite these added errors (see Ref. [31]). It would be beneficial to run these simulations while simultaneously accounting for space charge effects. The placement of BPMs in the OSC bypass must also be considered and their positions finalized. Another lattice correction study similar to [20] must be carried out for the OSC experiment setup once the lattice is finalized. 


\section{APPENDIX A}

\section{CHARACTERISTIC RAYS IN A MAGNETIC FIELD}


The trajectories of charged particles in an arbitrary static magnetic field making small angles with respect to the central trajectory are characterized by three kinds of rays. These characteristic rays are substituted into the general differential equation of motion and are evaluated with a Green's function integral. The rays have the following geometry :

1. A cosine-like trajectory $C_{x}(s)$ in the bending plane, with initial conditions $C_{x}(0)=1 ; C_{x}^{\prime}(0)=0$ (Fig. A.1).

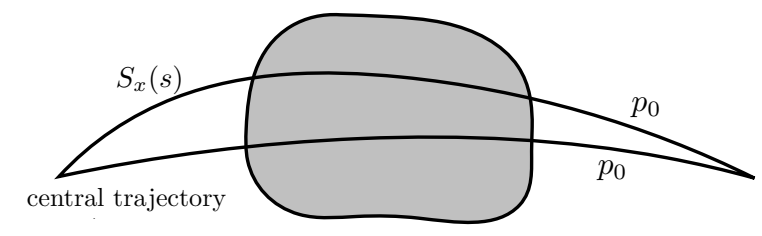

Figure A.1. The cosine-like trajectory $C_{x}(s)$ in the bending plane

2. A sine-like trajectory $S_{x}(s)$ in the bending plane, with initial conditions $S_{x}(0)=$ $0 ; S_{x}^{\prime}(0)=1$ (Fig. A.2).

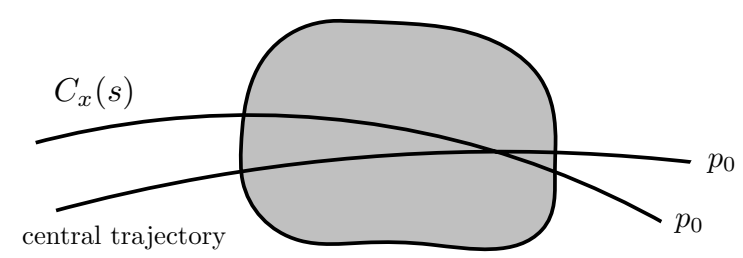

Figure A.2. The sine-like trajectory $S_{x}(s)$ in the bending plane

3. A dispersion-like trajectory $D_{x}(s)$ in the bending plane, with initial conditions $D_{x}(0)=0 ; D_{x}^{\prime}(0)=0$ (Fig. A.3). 


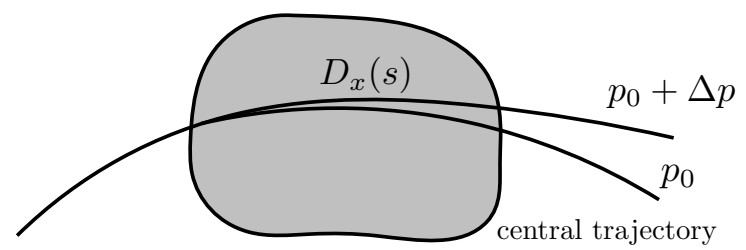

Figure A.3. The dispersion-like trajectory $D_{x}(s)$ in the bending plane 


\section{APPENDIX B}

PYTHON SCRIPT FOR THE IOTA GEOMETRY 
The geometric coordinates of each quadrupole and dipole can be outputted from MadX. A python script is used to take those coordinates and visually lay the ring with the quadrupoles in place and to scale. It is thereby easy to see if any elements interfere with each other, if the ring closes on itself, and the dimensions of the ring are readily extracted. Figure B.1 shows the output of the PYTHON script. The power-supply circuits and dipoles are hand-drawn on top of the ring.

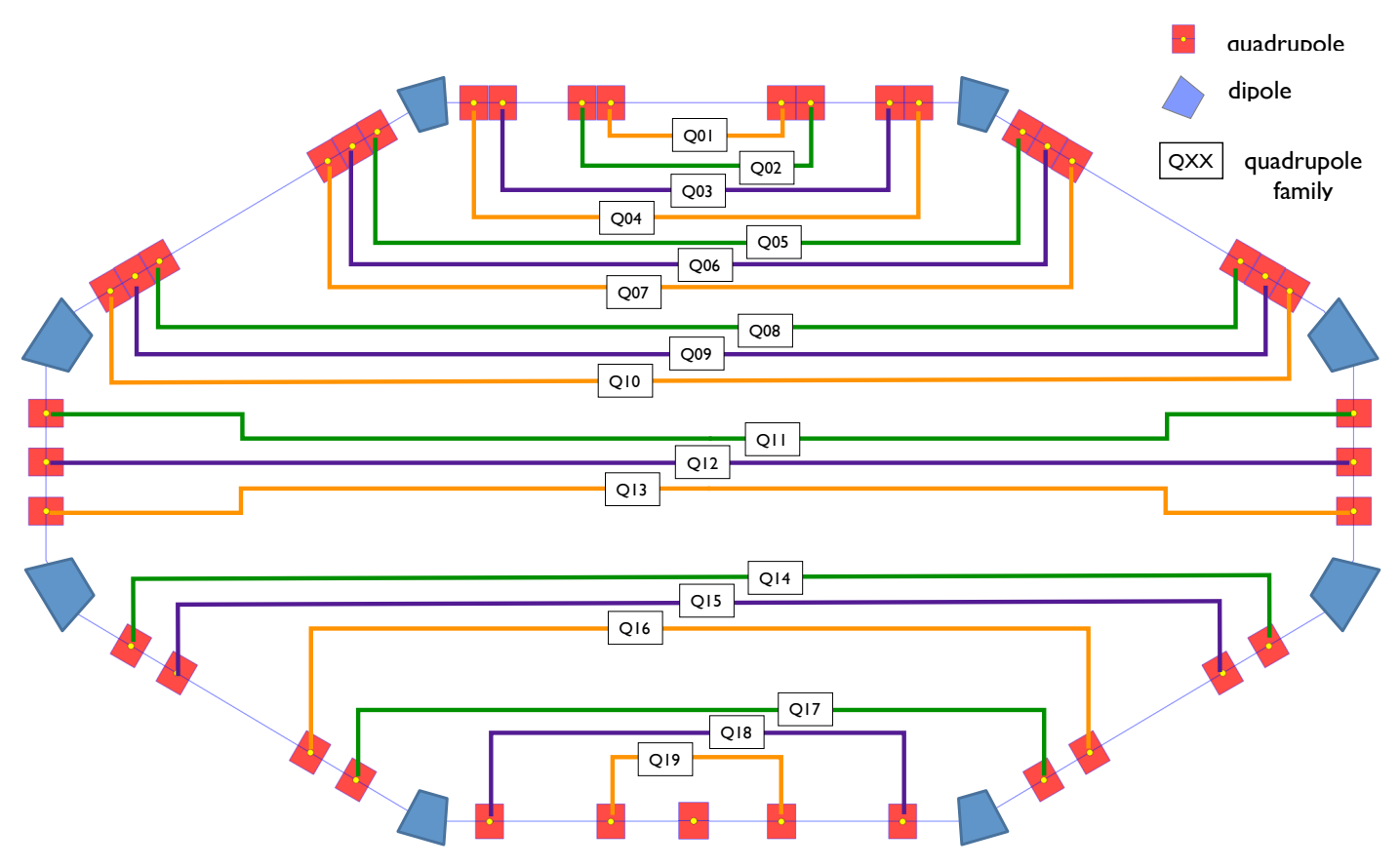

Figure B.1. The IOTA ring layout generated with Python. Dipoles (blue) are drawn in along with the quadrupole circuits.

The script utilizes the matplotlib. patches class to place and rotate the magnets in the ring, which in Figure B.1 are represented by rectangles.

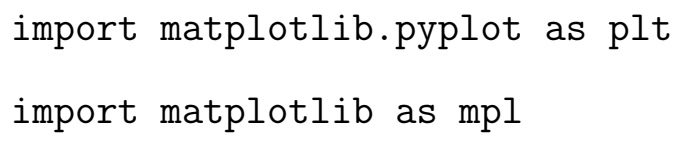




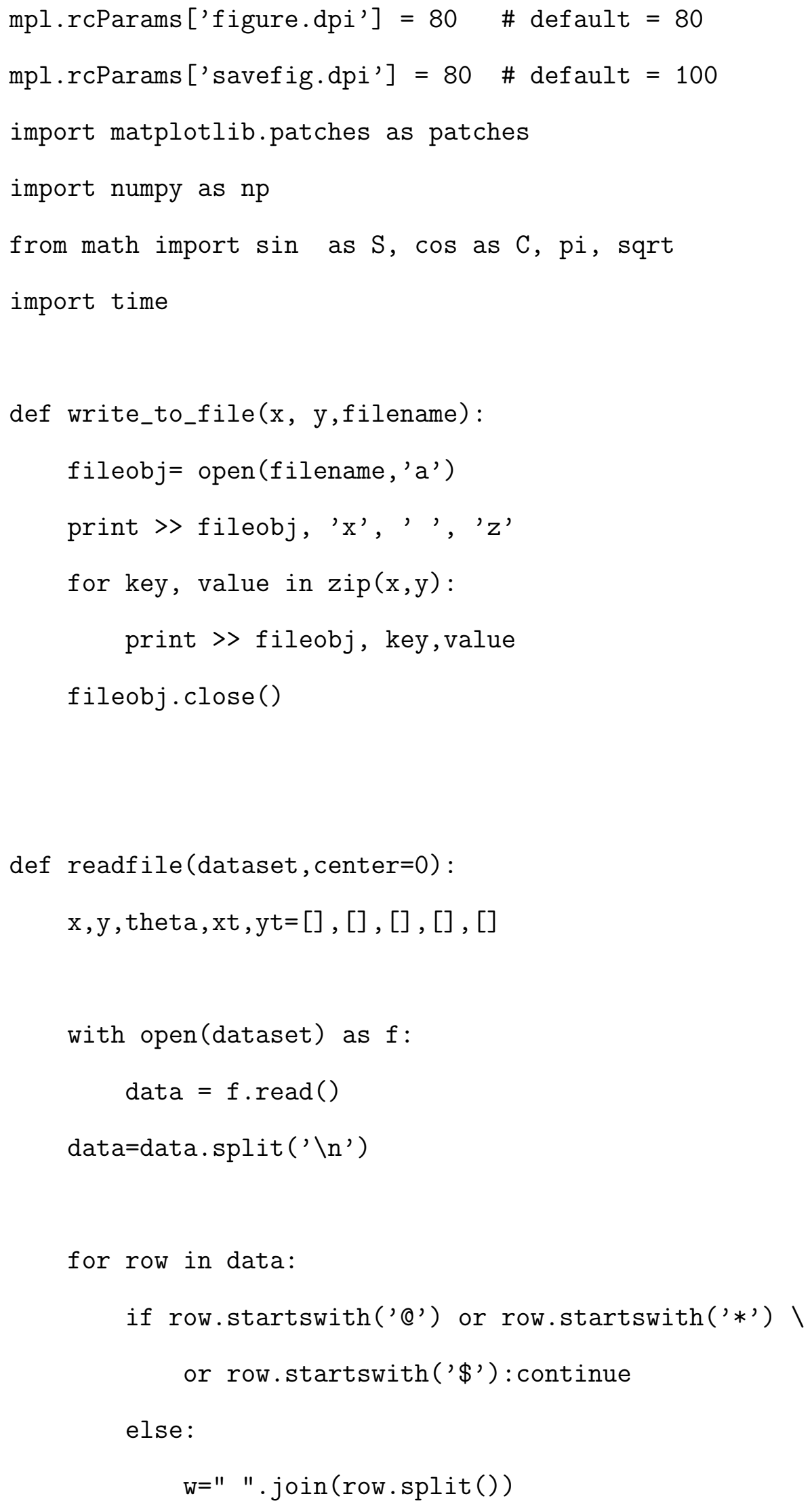




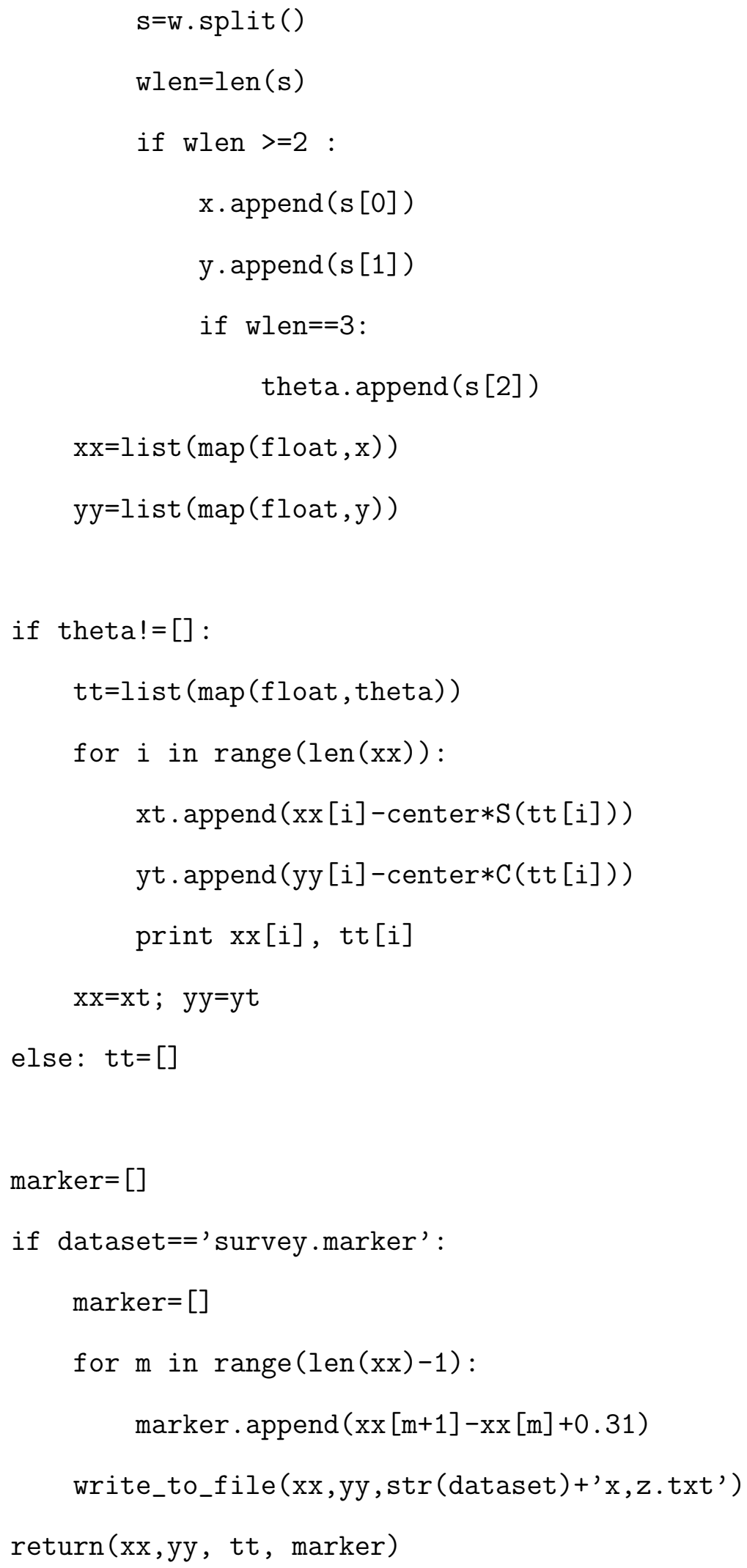




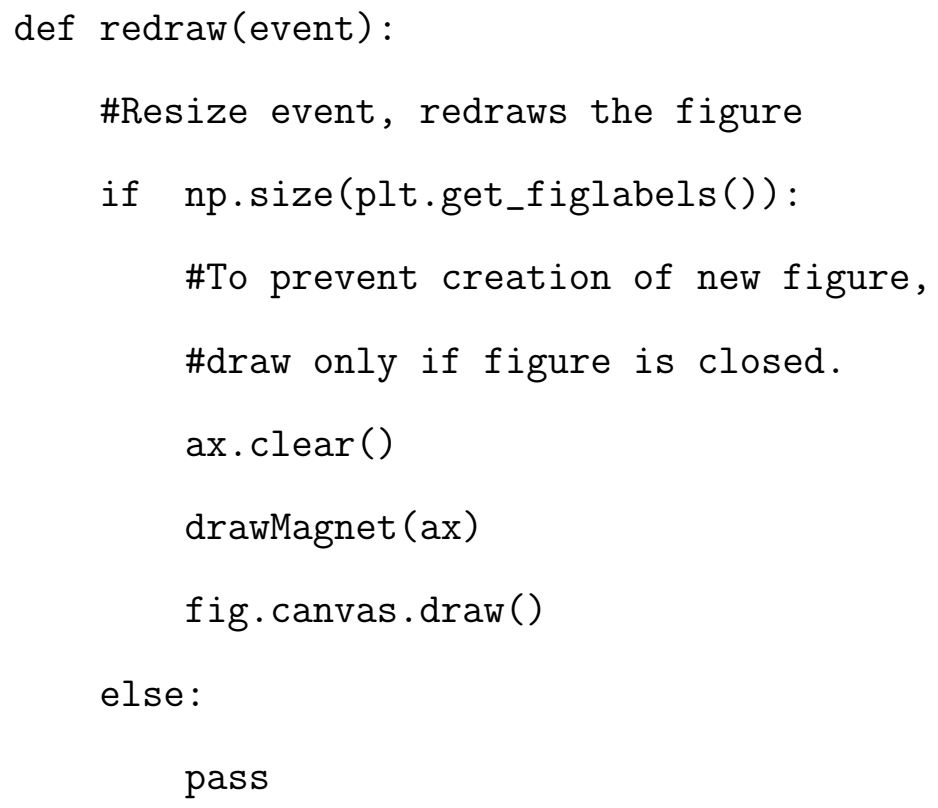

def drawMagnet(ax, $\mathrm{x}, \mathrm{y}$, theta, box_height, box_length, color): \#start of rectangular magnet 
plt.grid()

def set_canvas(width, height, x_min, x_max, y_min, y_max):

figSize $=$ (height, width)

fig = plt.figure('Patch rotate', figsize=figSize)

$\mathrm{ax}=$ fig.add_subplot(111)

ax.set_xlim(x_min, $\left.x_{-} \max \right)$

ax.set_ylim(y_min, y_max)

fig.canvas.mpl_connect ('resize_event', redraw)

ax.plot ( $f[1], f[2]$, color='red', markersize=30)

return fig 
APPENDIX C

ENVELOPE QUANTITIES AND MATRIX ELEMENTS 
The matched ellipse is generated with the ring's eigenvectors outputted from MadX. Knowing the emittance and the eigenvectors gives us the beam distribution at a given location in the ring:

$$
\sigma_{\text {beam }}^{6 D}=V_{6 \times 6} \epsilon_{6 \times 6} V_{6 \times 6}^{T}
$$

In order to generate a longitudinal emittance, we must first enable synchrotron radiation; this is done with the following commands:

beam, PARTICLE=electron, ENERGY=0 . 1005110034, RADIATE;

emit;

here, we have specified a $100 \mathrm{MeV}$ electron beam. The RF cavity must also be defined in the geometry file (where all the accelerator components are defined and placed). The RF cavity is called in MadX in the following manner:

RF : RFCAVITY, VOLT $=0.0001, L=0, H A R M O N=4, L A G=0$;

where the voltage is given in $\mathrm{MeV}$, the RF frequency is determined by the harmonic number and revolution frequency (the latter is calculated by MadX), and the "lag" is the phase lag. It is not necessary to specify a physical length for the RF cavity - here a thin gap of zero length gives the equivalent longitudinal kick. The momentum spread, together with synchrotron radiation and the placement of an RF cavity generates the appropriate 6D eigenvalues. The 36 eigenvalues are obtained using following code inside MadX's PTC module:

PTC_CREATE_UNIVERSE;

PTC_CREATE_LAYOUT, model $=2$, method $=6$, nst $=3$, exact ;

ptc_setswitch, debuglevel=0, exact_mis=true, time=true, totalpath=false; select,flag=ptc_twiss, clear; 


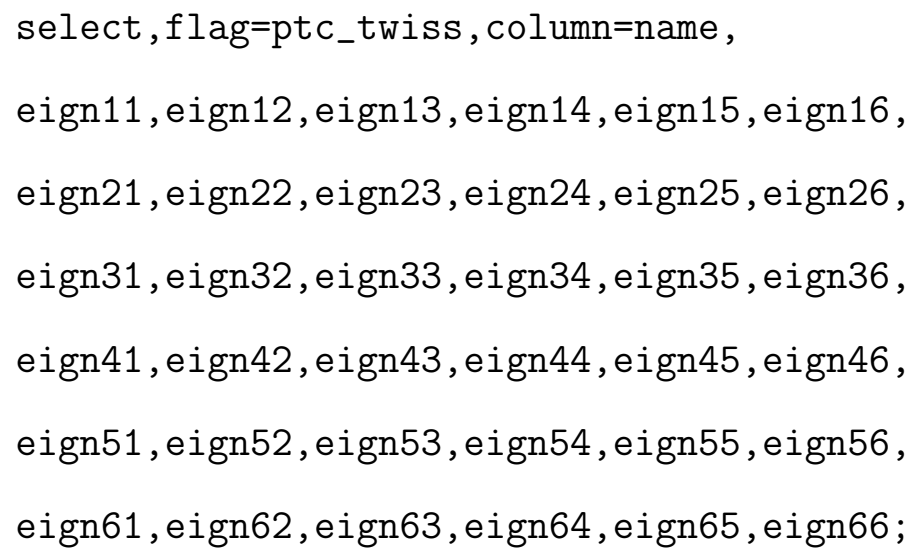

Here, eignij is the eigenvalue in the $i$ th row and $j$ th column of the $\sigma_{\text {beam }}^{6 D}$ matrix. Once this matrix is determined, the random gaussian distribution is generated using Python's "multivariate normal" function from the Numpy library:

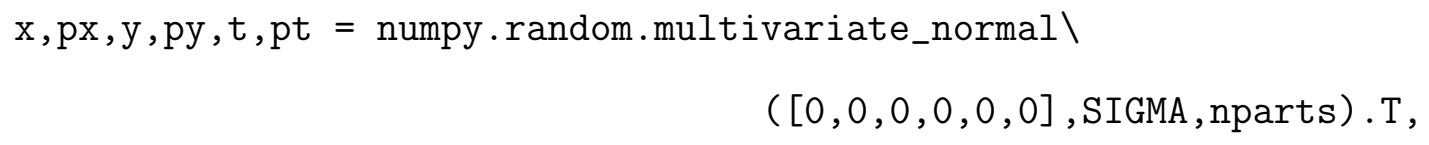

where the null vector denotes a beam that is not offset, "SIGMA" is the $6 \times 6$ beam matrix, and "nparts" is the number of particles in the distribution. The output is a list of particles each with 6 phase space coordinates.

In order to create a $2 \mathrm{D}$ transverse cut on a beam at a given number of standard deviations, we need to extrapolate the standard equation for an ellipse from the horizontal components in equation 2.69. We can write the equation of an ellipse as the following [32]:

$$
\sigma_{11} \sigma_{22}-\sigma_{12}^{2}=\sigma_{22} x^{2}+\sigma_{11} x^{2}-2 \sigma_{12} x x^{\prime}
$$

where $\sigma_{i j}$ are the elements in the matrix $\Sigma_{\text {beam }}^{6 D}$ found in equation 2.69. The semimajor and minor axes of the ellipse are given by the following equations:

$$
A=\frac{1}{\sqrt{2}} \sqrt{\sigma_{11}+\sigma_{22}+\sqrt{\sigma_{11}^{2}+\sigma_{11}^{2}+4 \sigma_{12}^{2}-2 \sigma_{11}^{2} \sigma_{22}^{2}}}
$$




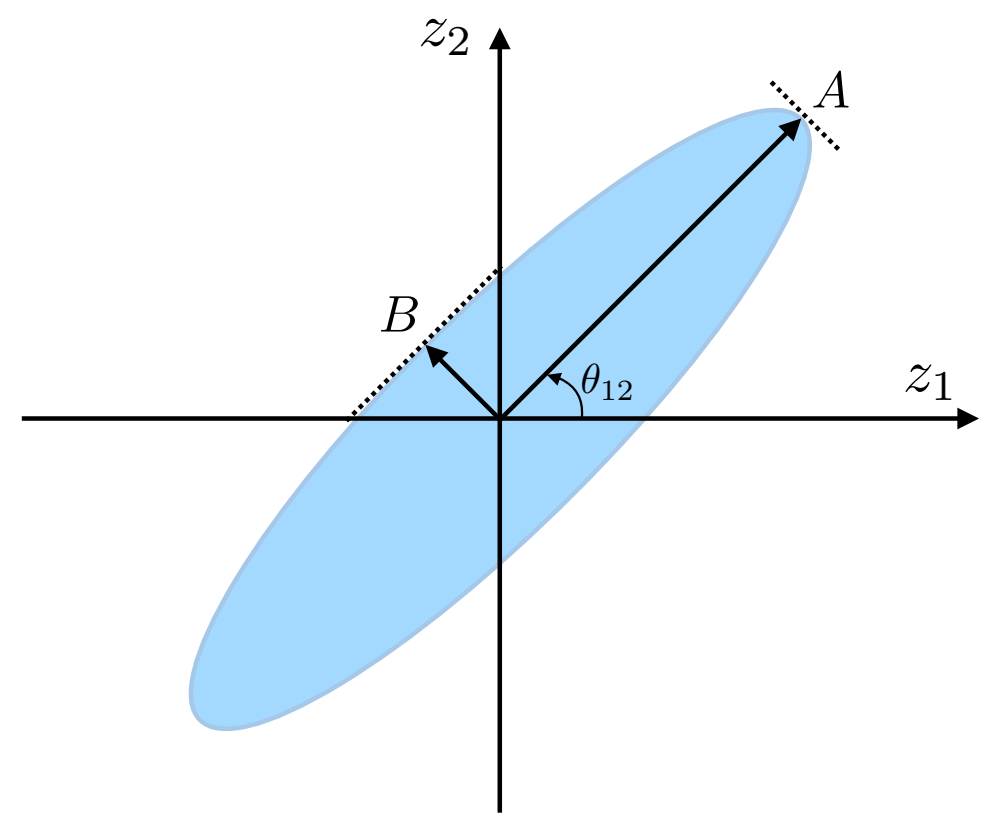

Figure C.1. The semi-major, $A$, and minor, $B$, axes of an ellipse tilted at an angle $\theta_{12}$ from the horizontal.

$$
\begin{gathered}
B=\frac{1}{\sqrt{2}} \sqrt{\sigma_{11}+\sigma_{22}-\sqrt{\sigma_{11}^{2}+\sigma_{11}^{2}+4 \sigma_{12}^{2}-2 \sigma_{11}^{2} \sigma_{22}^{2}}}, \\
\theta=\frac{1}{2} \arctan \left(\frac{2 \sigma_{12}}{\sigma_{11}-\sigma_{22}}\right) .
\end{gathered}
$$

Ellipses at 1,2 and $3 \sigma$ can be superimposed in the $x$-px plane and cuts can easily be made. For the purposes of the simulations in section 5, an initial distribution of 100,000 particles was made with the proper 6-dimensional coupling. Only particles at 1, 2 and 3 sigma were kept for the particle tracking as seen in Figure C.2. 


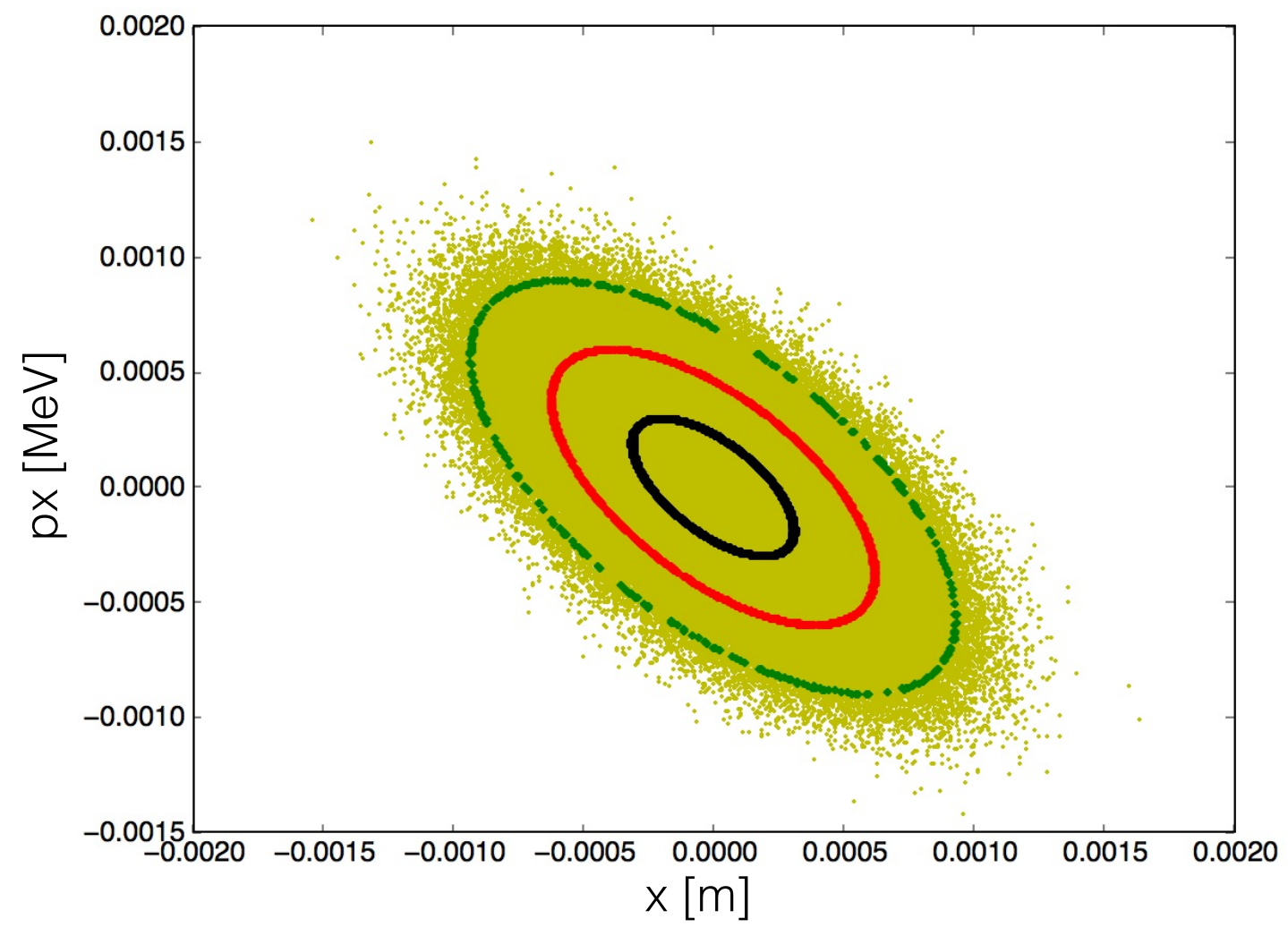

Figure C.2. A fully coupled beam is generated in 6 dimensions. A narrow band of particles at 1, 2 and $3 \sigma$ (black, red and green, respectively) are selected out in the $x-p x$ plane and are tracked through the bypass. The rest of the particles are discarded. 
APPENDIX D MADX/PTC SCRIPT FOR OSC BYPASS SEXTUPOLES 
The sextupole gradients in the OSC bypass were tuned using the PTC module in MadX. First a table is created and filled with the second order matrix element we wish to minimize:

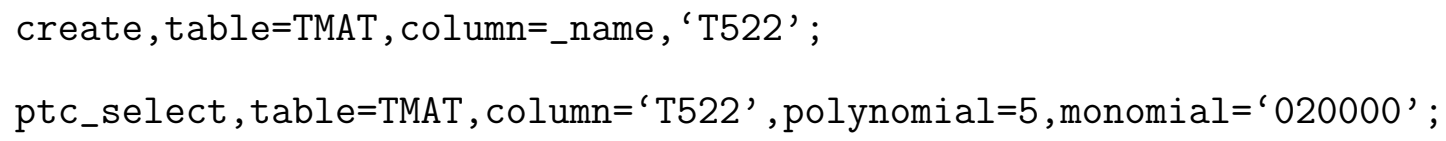

The second order matrix element indices $T_{i j k}$ are specified by polynomial=i and monomial $=j k$ and are stored in the table TMAT under column ' T522' . Next, a macro is defined in the PTC universe that specifies the section of the ring to be used in the tuning; here the section used is called bypass with its magnetic elements specified in another file:

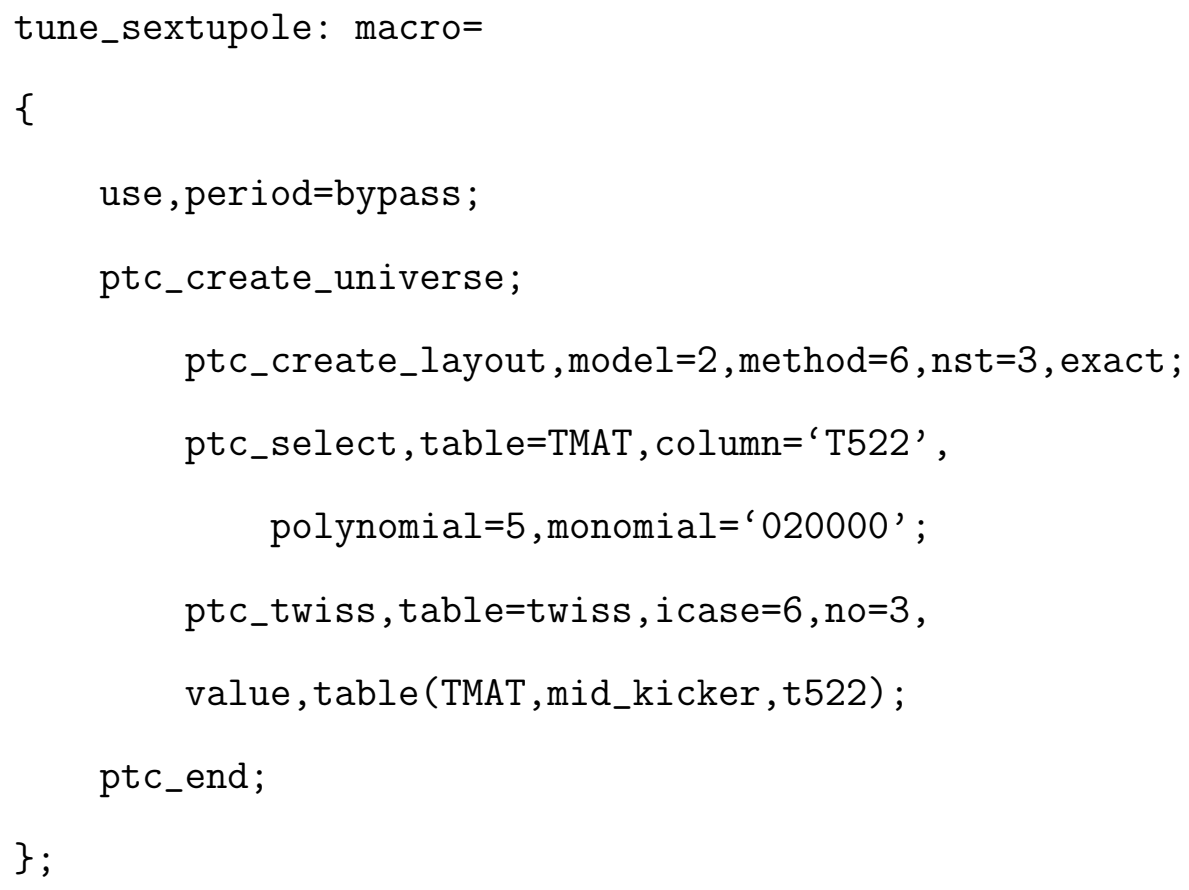

The model values describes a "Matrix-Kick-Matrix" type integration and the method and net specifies the type and number of integration steps; details can be found in the MadX manual [14]. Lastly, matching section defines the parameter to change along with the constraint, or goal function to achieve: 
match, use_macro;

vary, name $=\mathrm{s} 1->\mathrm{k} 2$, step $=0.0001$

use_macro, name=tune_sextupole;

constraint, expr=table(TMAT, mid_kicker, T522) =0;

value, expr;

jacobian, tolerance $=1 \mathrm{e}-20, \operatorname{calls}=1000$;

Since the sextupoles in the bypass are identical and are described by the same name, the function s1-> k2 points to both sextupole gradients, and are adjusted simultaneously. The constraint specifies the element at which the goal function must attain the given value; here it is the midpoint in the kicker undulator mid_kicker. 


\section{APPENDIX E}

PYTHON/MADX SIMULATION FOR OSC 
The following python script was used to demonstrate OSC in the IOTA ring. It is designed to run on multiple processors to cut down on simulation time for many particles. It generates particles with the appropriate $6 \mathrm{D}$ coordinates and then tracks them through the bypass applying appropriate kicks, and then passes them through the rest of the ring. The number of processors, particles and turns around the ring can be easily changed in the "main" function. The output of the simulation is a phase space diagram of the longitudinal coordinates at the end of the chicane after every turn around the ring.

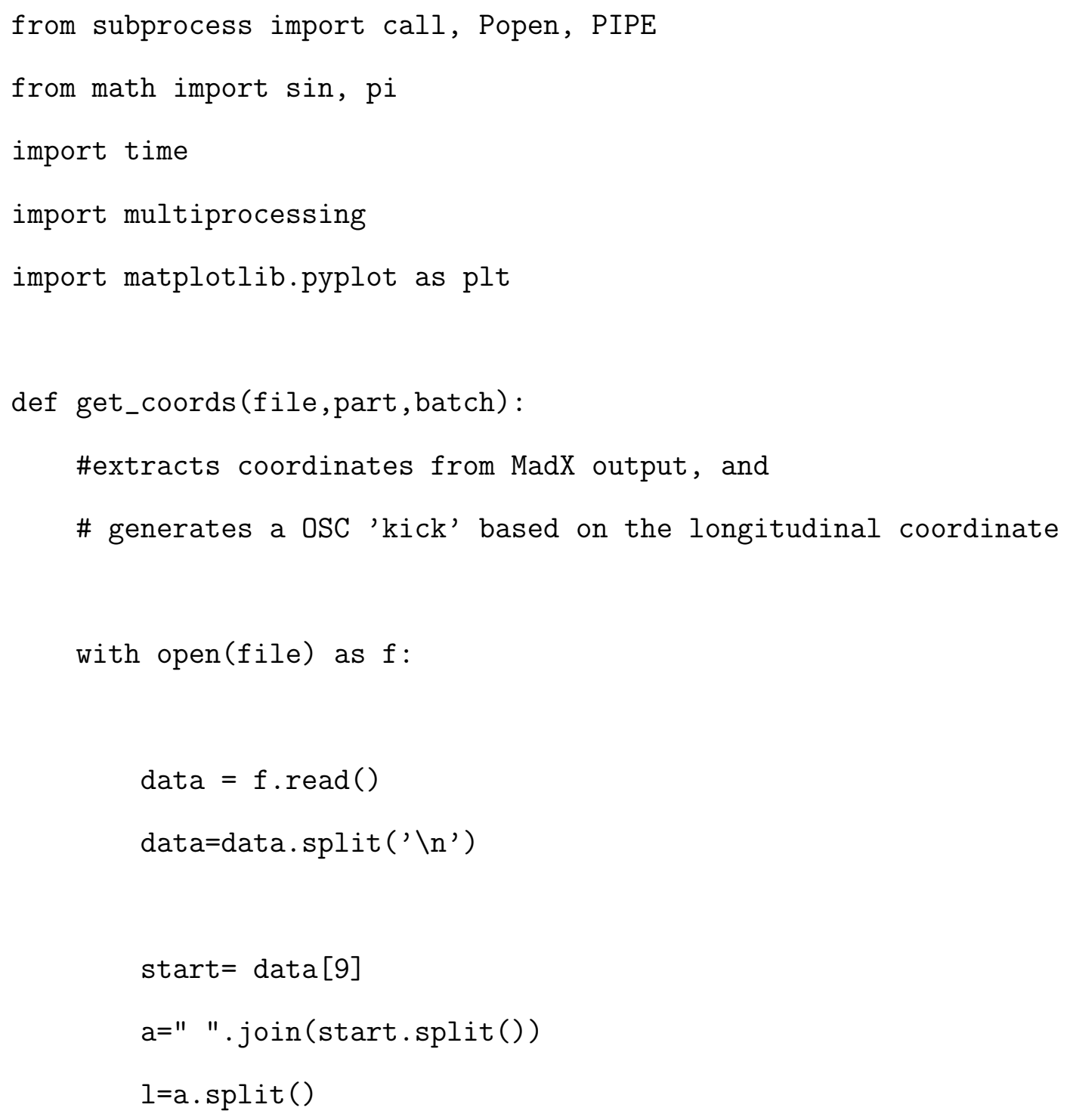




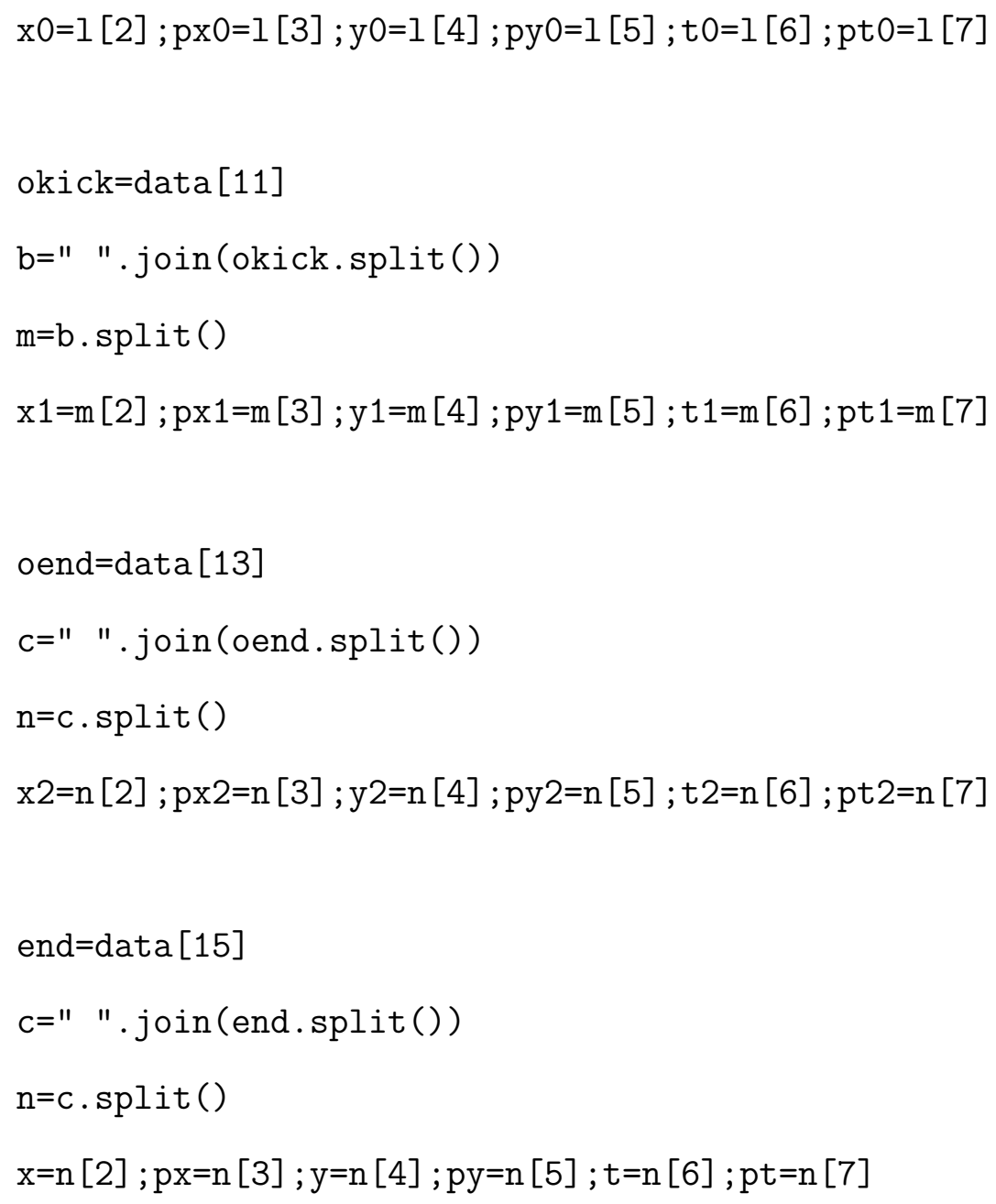

$\# d z$ is how far the particle falls or exceeds \# the reference particle longitudinally. $d z=f$ loat $(t 1)-f$ loat $(t 0)$

\#the magnitude and phase of the kick is determined prior; \#the offset ' $\mathrm{dz}$ ' is what changes on each successive turn $\mathrm{dE}=-0.6 * \sin (\mathrm{dz} * 2 * \mathrm{pi} * 0.05 \mathrm{e} 6)$

return $\mathrm{x} 0, \mathrm{px} 0, \mathrm{y} 0, \mathrm{py} 0, \mathrm{t} 0, \mathrm{pt} 0, \mathrm{x} 1, \mathrm{px} 1, \mathrm{y} 1, \mathrm{py} 1, \mathrm{t} 1, \mathrm{pt} 1, \backslash$ $\mathrm{x} 2, \mathrm{px} 2, \mathrm{y} 2, \mathrm{py} 2, \mathrm{t} 2, \mathrm{pt} 2, \mathrm{x}, \mathrm{px}, \mathrm{y}, \mathrm{py}, \mathrm{t}, \mathrm{pt}, \mathrm{dE}, \mathrm{dz}$, part, batch 


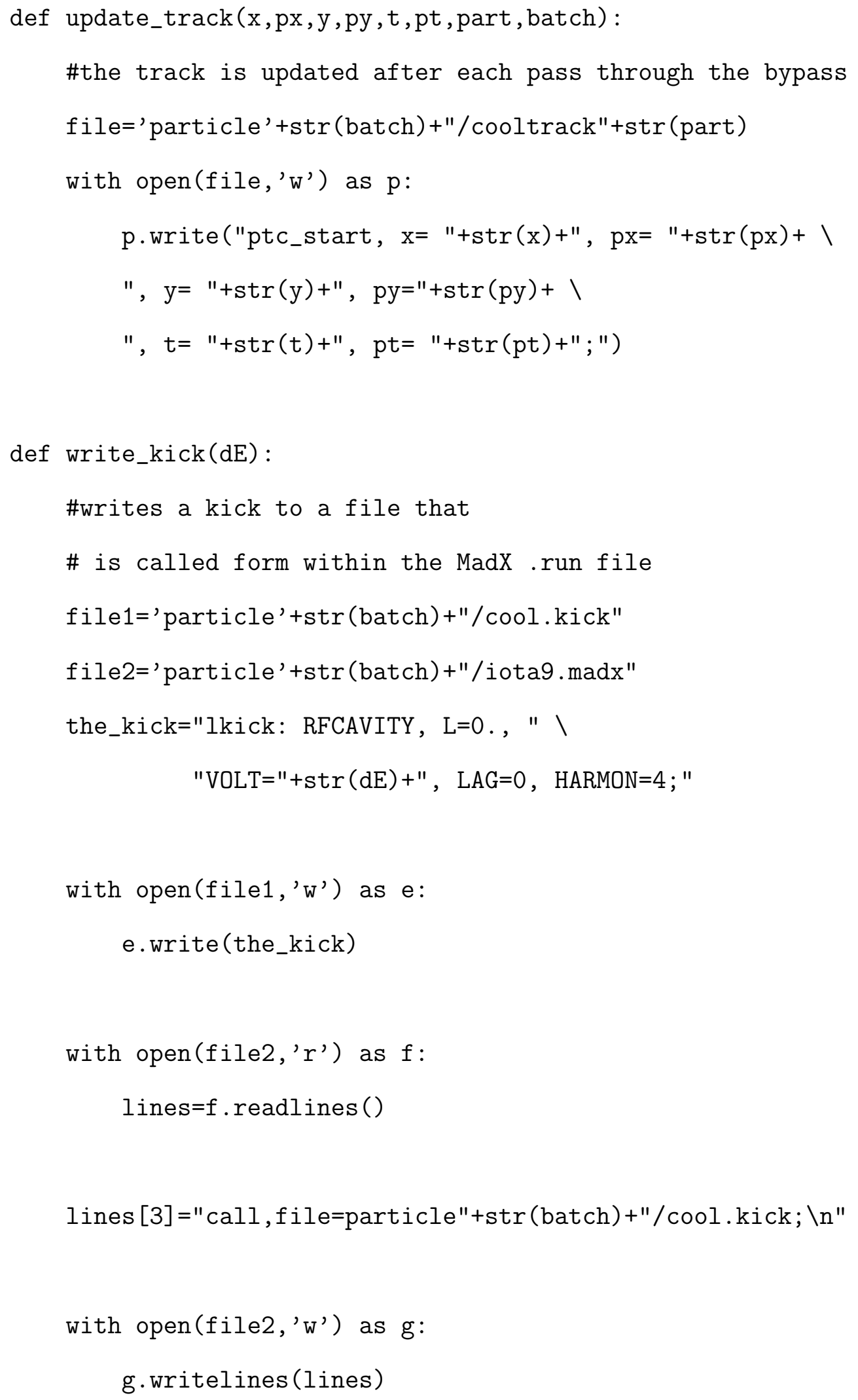




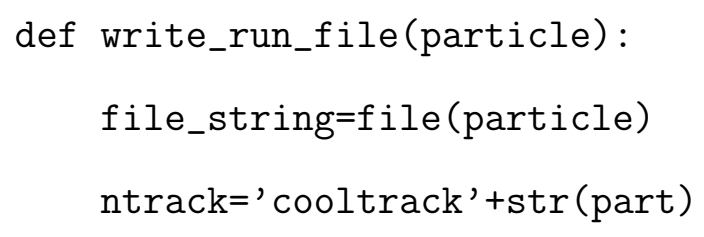

with open('particle'+str(batch)+'/cool.run', 'w') as 0 : \#data $=0$.readlines () data $=$ file_string o.writelines (data)

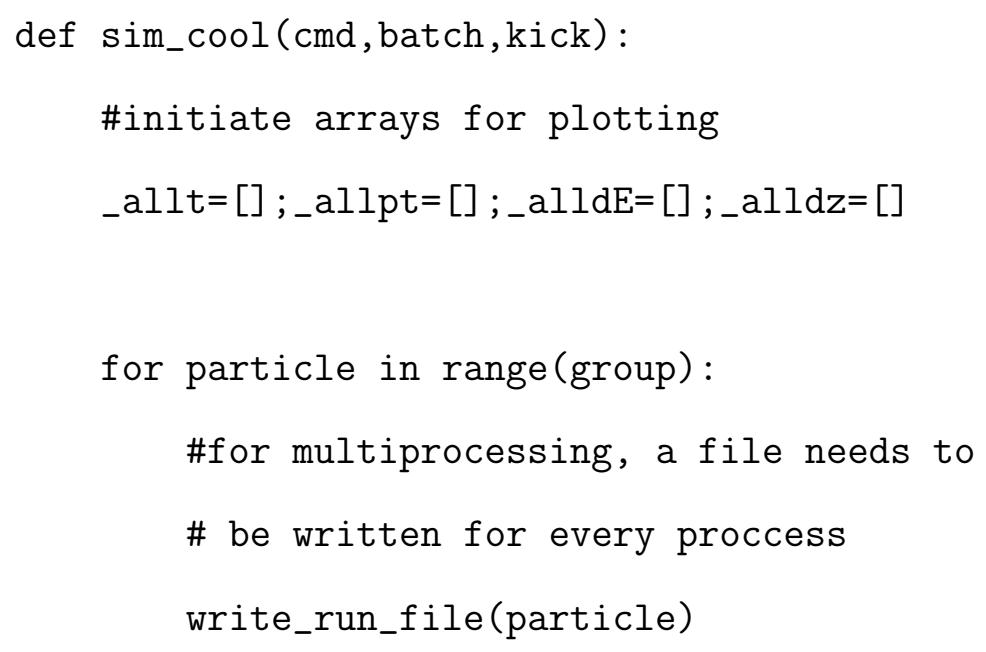




$$
\text { str (batch)+'/trackone', particle, batch) }
$$

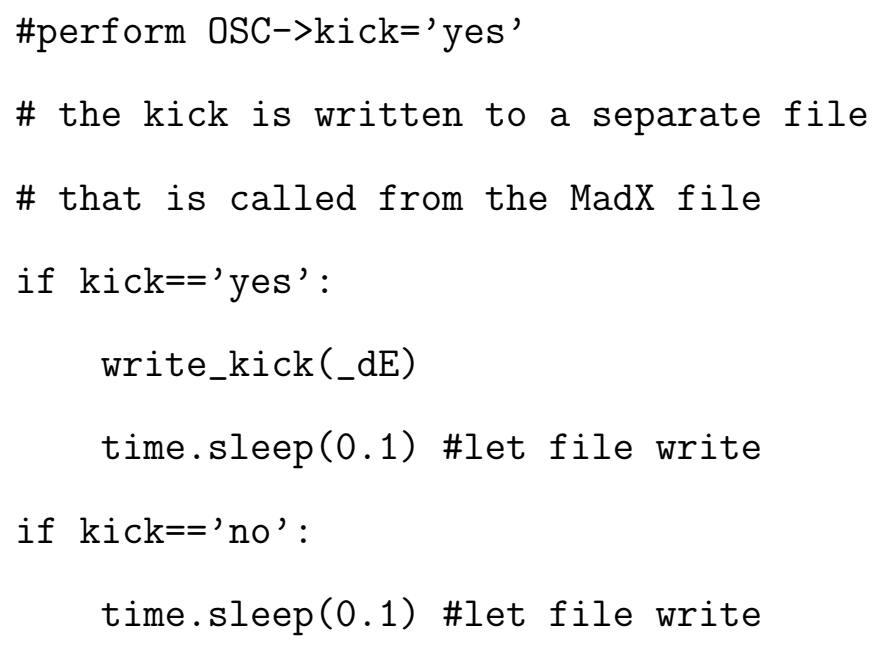

\#run particle through byapss

launch=Popen (cmd, shell=True, stdout=PIPE)

launch.wait() \#let MadX finish before continuing

\#get particle coordinates at end of bypass

$\mathrm{xP}_{-}, \mathrm{pxP}_{-}, \mathrm{yP}_{-}, \mathrm{pyP}_{-}, \mathrm{tP} \mathrm{P}_{-}, \mathrm{ptP}_{-}, \mathrm{xK}_{-}, \mathrm{pxK}_{-}, \backslash$

$\mathrm{yK}_{-}, \mathrm{pyK}_{-}, \mathrm{tK}_{-}, \mathrm{ptK}_{-}, \mathrm{xL}_{-}, \mathrm{pxL}_{-}, \mathrm{yL}_{-}, \mathrm{pyL}_{-}, \mathrm{tL}_{-}, \mathrm{ptL}_{-}, \backslash$

$\mathrm{x}_{-}, \mathrm{px}_{-}, \mathrm{y}_{-}, \mathrm{py}_{-}, \mathrm{t}_{-}, \mathrm{pt}_{-}, \mathrm{dE}_{-}, \mathrm{dz}_{-}, \mathrm{part}_{-}, \mathrm{batch}_{-}=\backslash$

get_coords ('particle'+str(batch)+

'/trackone', particle, batch)

\#update the coordinate extracted above update_track $\left(\mathrm{x}_{-}, \mathrm{px}_{-}, \mathrm{y}_{-}, \mathrm{py}_{-}, \mathrm{t}_{-}, \mathrm{pt} \mathrm{t}_{-}\right.$, part $\mathrm{f}_{-}$, batch $\left.)\right)$

\#put coordinates in array for plotting _allt.append (float (tL_)) 


$$
\begin{aligned}
& \text { _allpt.append (float } \left.\left(\mathrm{ptL}_{-}\right)\right) \\
& \text {_alldz.append (float } \left.\left(\mathrm{dz}_{-}\right)\right) \\
& \text {_alldE. append (float } \left.\left(\mathrm{dE}_{-}\right)\right)
\end{aligned}
$$

\#plot the new coordinate and leave the \# figure open until all turns are completed plt.plot(_alldz,_alldE, 'o', markersize $=5$, color $=$ ' black')

plt.savefig( 'dE_dz_' +str (batch)+' .png')

plt.close()

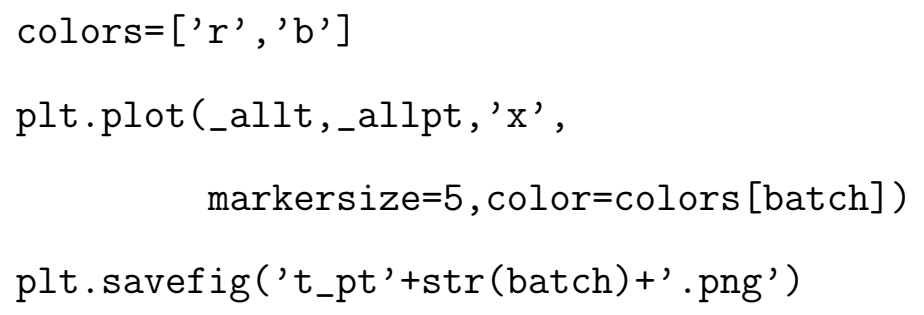




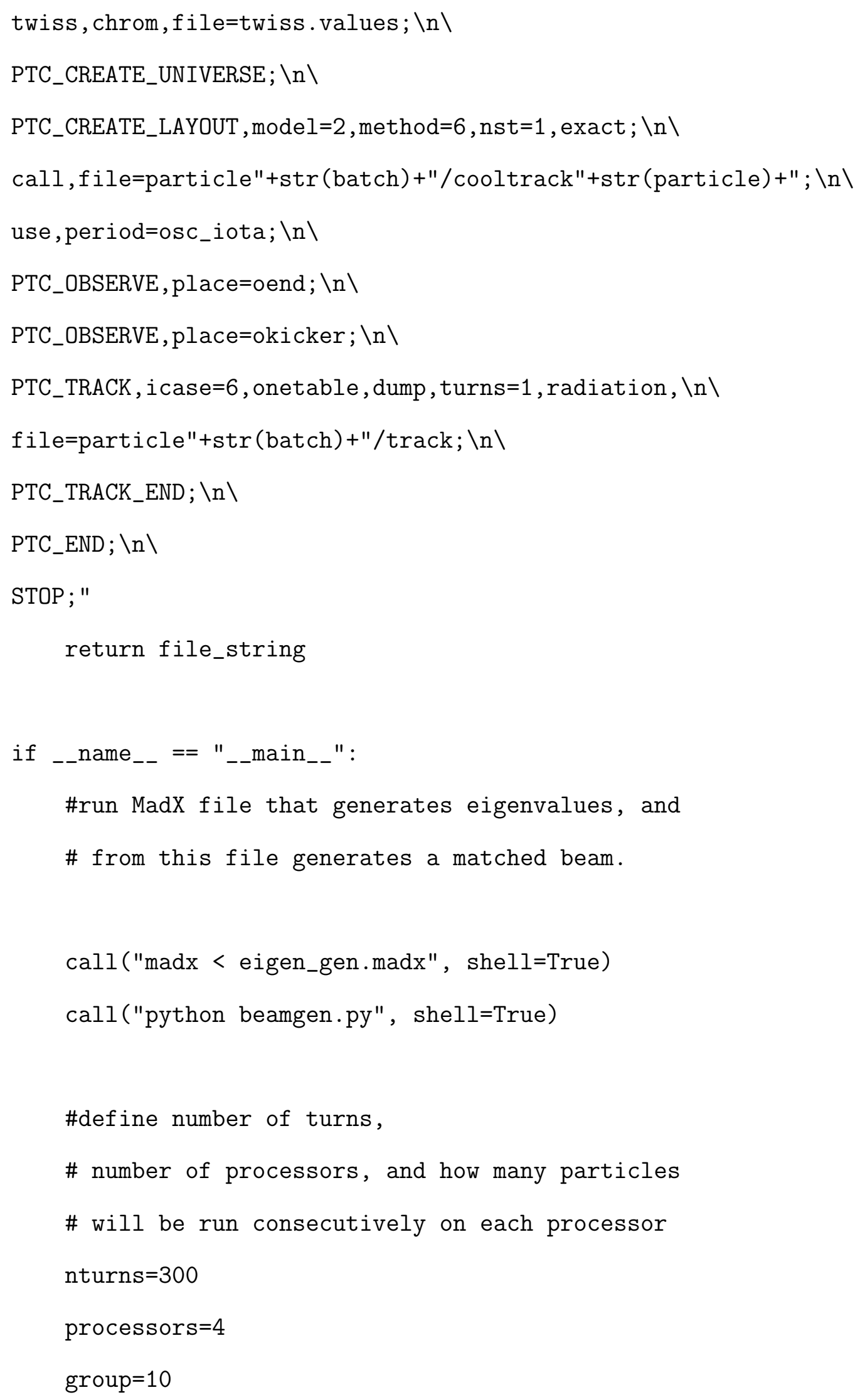


kickit='yes'

for batch in range(processors):

\#define some strings for writing files

num $=1 \% \mathrm{~s} " \%$ batch

part $=" \operatorname{cool} "+$ num

cmd $=$ "madx $<"$ +'particle'+num+"/cool.run"

\#start the simulation!

process $=$ multiprocessing.Process $($ target $=$ sim_cool, $\operatorname{args}=(\mathrm{cmd}$, batch, kickit $))$

process.start() 


\section{BIBLIOGRAPHY}

[1] M. Church, H. Edwards, P. Garbincius, E. Harms, S. Henderson, S. Holmes, A. Lumpkin, R. Kephart, V. Lebedev, J. L. S. Nagaitsev, P. Piot, C. Prokop, V. Shiltsev, Y. Sun, A. Valishev, R. Abrams, G. Allen, C. Ankenbrandt, D. Arnold, B. Beaudoin, S. Biedron, B. Blomberg, S. Boucher, C. Brau, D. Bruhwiler, J. Byrd, L. Cambell, B. Carlsten, B. Chase, D. Christian, M. Chung, J. Corlett, M. Cummings, V. Danilov, Y. Derbenev, B. Digiovine, N. Eddy, P. Emma, B. Erdelyi, J. Fast, G. Flanagan, A. Garraud, A. Grabenhofer, D. Henderson, R. Holt, P. Hurh, R. Johnson, G. Kazakevitch, Y. Kim, R. Kishek, T. Koeth, G. Krafft, F. Lemery, A. Malyzhenkov, A. Marchionni, D. Mihalcea, S. Milton, A. Morin, A. Murokh, E. Nissen, M. Palmer, B. Peterson, E. Prebys, J. Qiang, K. Rehm, M. Reinsch, T. Roberts, A. Robinson, J. Ruan, K. Ruisard, V. Scarpine, T. Sen, Y. Shin, E. Simakov, N. Solyak, A. Sonnenschein, D. Still, R. Suleiman, Y. Sun, D. Sutter, J. Thangaraj, R. Thurman-Keup, Y. Tokpanov, R. Tschirhart, C. Ugalde, M. Venturini, N. Vinokurov, R. Wilcox, T. Xu, V. Yakovlev, N. Yampolsky, Y. Zhang, T. Zolkin, M. Zolotorev, and R. Zwaska, "Proposal for an Accelerator R\&D User Facility at Fermilab's Advanced Superconducting Test Accelerator (ASTA)," FERMILAB-TM-2568, 2013.

[2] L. Landau, "On the vibrations of the electronic plasma," J.Phys.(USSR), vol. 10, pp. 25-34, 1946.

[3] L. Palumbo and
particle M. $\begin{gathered}\text { Migliorati, } \\ \text { (draft)," }\end{gathered}$ accelerators tor Physics Course, Chios, Greece, September 2011. https://indico.cern.ch/event/115334/contribution/31/material/paper/0.pdf.

[4] S. van der Meer, "Stochastic damping of betatron oscillations in the ISR," Tech. Rep. CERN-ISR-PO-72-31. ISR-PO-72-31, CERN, Geneva, August 1972.

[5] K. L. Brown, "A first and second order matrix theory for the design of beam transport systems and charged particle spectrometers," Technical Report SLAC75, June 1982.

[6] D. C. Carey, The Optics of Charged Particles. Chur, Switzerland: Harwood Academic Publishers, 1987.

[7] H. Wiedemann, Particle Accelerator Physics. 3rd ed. New York: Springer, 1993.

[8] J. D. Jackson, Classical Electrodynamics. 3rd ed. New York: John Wiley \& Sons, Inc., 1999.

[9] D. Möhl, "Beam cooling," in CERN Accelerator School: Basic Course on General Accelerator Physics, Loutraki, Greece, pp. 224-239, October 2000.

[10] A. Wolski, "The accelerator physics of linear collider damping rings," USPAS Lecture, 2003, www.desy.de/njwalker/uspas/coursemat/notes/unit4notes.pdf.

[11] J. L. Duff, "Single and multiple Touschek effects," CERN Accelerator School: Second Advanced Accelerator Physics Course, pp. 114-122, September 1987.

[12] V. Lebedev, "Single and multiple intrabeam scattering in hadron colliders," in AIP Conf. Proc., vol. 773, pp. 440-442, 33rd ICFA Advanced Beam Dynamics Workshop on High-Intensity and High-Brightness Hadron Beams (HB2004), 2005. 
[13] F. Willeke and G. Ripken, "Methods of Beam Optics," in Physics of Particle Accelerators, AIP Conf. Proc., vol. 184, pp. 758-819, 1989.

[14] V. Kapin, Y. Alexahin, and F. Schmidt, Using Eigenvectors as Constraints in MadX Matching Module, 2006. http://beamdocs.fnal.gov/AD/DocDB/0024/ 002449/002/20061013_Report.pdf.

[15] V. Lebedev and S. A. Bogacz, "Betatron motion with coupling of horizontal and vertical degrees of freedom," JINST, vol. 5, P10010, pp. 1-24, 2010.

[16] S. Nagaitsev and V. Danilov, "A search for integrable four-dimensional nonlinear accelerator lattices," in Proceedings of IPAC'10, THPE094, pp. 4743-4745, 1st International Particle Accelerator Conference: IPAC'10. 23-28 May 2010. Kyoto, Japan, 2010.

[17] G. Darboux, "Sur un probléme de mécanique," Arch. Neerl. Sci., vol. 6, pp. 371376, 1901.

[18] F. H. O'Shea, R. Agustsson, A. Murokh, E. Spranza, S. Nagaitsev, and A. Valishev, "Measurement of non-linear insert magnets," in Proceedings of PAC2013, Pasadena, CA, USA, WEPBA17, pp. 922-924, 2013.

[19] S. Nagaitsev, A. Valishev, V. Danilov, and D. Shatilov, "Beam Physics of Integrable Optics Test Accelerator at Fermilab," in Proceedings of IPAC2012, New Orleans, Louisiana, USA, TUPPC090, pp. 1371-1373, 3rd International Particle Accelerator Conference, 2012.

[20] A. Romanov, G. Kafka, S. Nagaitsev, and A. Valishev, "Lattice Correction Modeling for Fermilab IOTA Ring," in Proceedings of IPAC2014, TUPRO058, pp. 1165 - 1167, 5th International Particle Accelerator Conference, Dresden Germany, 2014.

[21] V. Lebedev, "OptiM." http://www-bdnew.fnal.gov/pbar/ organizationalchart/lebedev/OptiM/optim.htm, 2008.

[22] G. Stancari, "Applications of electron lenses: scraping of high-power beams, beam-beam compensation, and nonlinear optics," Proceedings of the 16th Advanced Accelerator Concepts Workshop (AAC 2014), 2014 (in press), arXiv: physics.acc-ph/1409.3615 (pre-print).

[23] Y. Li, "Beam manipulation using lasers," in Lecture 6, U.S. Particle Accelerator School: Laser Applications to Accelerators (University of Maryland), June 2008.

[24] M. Zolotorev and A. A. Zholents, "Transit-time method of optical stochastic cooling," Physical Review E, vol. 50, no. 4, pp. 3087-3091, 1994.

[25] V. Lebedev, "Optical stochastic cooling in Tevatron," 46th ICFA Advanced Beam Dynamics Workshop on High-Intensity and High-Brightness Hadron Beams HB2010, Sep 27 - Oct 1 2010. Morschach, Switzerland, pp. 644-646, 2010.

[26] V. Lebedev, Y. Tokpanov, and M. Zolotorev, "Test of Optical Stochastic Cooling in the IOTA Ring," in North American Particle Accelerator Conference (PAC 2013), TUODA2, pp. 422-424, 2013.

[27] K. W. Robinson, "Radiation effects in circular electron accelerators," Phys. Rev., vol. 111, no. 2, pp. 373-380, 1958. 
[28] G. Guignard and J. Hagel, "Sextupole correction and dynamic aperture: Numerical and analytical tools," Particle Accelerators, vol. 18, pp. 129-165, 1986.

[29] V. Lebedev, "Approximating sextupole correction." Meeting at Fermilab (unpublished), April 2014.

[30] Y. Li, W. Deeking, B. Faatz, and J. Pflueger, "Microbunch preserving bending system for a helical radiator at the European x-ray free electron laser," Physical Review Special Topics - Accelerators and Beams, vol. 13, 080705, pp. 1-12, August 2010.

[31] S. Chattopadhyay, C. Kim, D. J. Massoletti, A. A. Zholents, M. S. Zolotorev, and W. Wan, "Study on a test of optical stochastic cooling scheme in a single pass beam line," Tech. Rep. CBP-203. LBNL-39788, Lawrence Berkeley Nat. Lab., Berkeley, CA, January 1997.

[32] R. J. Bhatt, Inverse Problems in Elliptic Charged-Particle Beams. PhD thesis, Massachusetts Institute of Technology, Cambridge, Massachusetts, June 2006. 